TRANSACTIONS OF THE

AMERICAN MATHEMATICAL SOCIETY

Volume 358, Number 1, Pages 403-439

S 0002-9947(05)03795-5

Article electronically published on July 26,2005

\title{
QUASI-FINITE MODULES FOR LIE SUPERALGEBRAS OF INFINITE RANK
}

\author{
NGAU LAM AND R. B. ZHANG
}

\begin{abstract}
We classify the quasi-finite irreducible highest weight modules over the infinite rank Lie superalgebras $\widehat{g l}_{\infty \mid \infty}, \widehat{\mathrm{C}}$ and $\widehat{\mathcal{D}}$, and determine the necessary and sufficient conditions for such modules to be unitarizable. The unitarizable irreducible modules are constructed in terms of Fock spaces of free quantum fields, and explicit formulae for their formal characters are also obtained by investigating Howe dualities between the infinite rank Lie superalgebras and classical Lie groups.
\end{abstract}

\section{INTRODUCTION}

Supersymmetry permeated many areas of mathematics in the last decade, producing deep results such as Seiberg-Witten theory and mirror symmetry. In most applications, supersymmetry manifests itself in concrete representations of the relevant Lie superalgebras [15. This makes it particularly important to develop the representation theory of Lie superalgebras.

In this paper we investigate the representation theory of Lie superalgebras $\widehat{\mathrm{gl}}_{\infty \mid \infty}$ and its osp-type Lie subsuperalgebras $\widehat{\mathcal{C}}$ and $\widehat{\mathcal{D}}$ (see Section 3 for their definitions). (Aspects of $\widehat{\mathcal{B}}$ were studied in [7].) These Lie superalgebras are a class of $\frac{1}{2} \mathbb{Z}$-graded infinite rank Lie superalgebras arising from central extensions of Lie superalgebras of complex matrices of infinite size. They were featured very prominently in the study [7] of the super $W_{1+\infty}$ algebra, and were shown [3] to be intimately related to affine Kac-Moody superalgebras. Both the $W_{1+\infty}$ superalgebra and affine Kac-Moody superalgebras play fundamental roles in conformal filed theory and superstrings.

Recall that the infinite dimensional Lie algebra $\widehat{\mathrm{gl}}_{\infty}$ and its various subalgebras were extensively studied in [17, 18, 24, 25] in relation to $W_{1+\infty}$. In particular, the notion of quasi-finite modules [17] over infinite dimensional graded Lie (super)algebras were introduced. Such modules are close to finite dimensional representations of finite dimensional Lie (super)algebras in spirit. In our context, a

Received by the editors October 30, 2003 and, in revised form, June 11, 2004.

2000 Mathematics Subject Classification. Primary 17B65, 17B10.

Key words and phrases. Infinite rank Lie superalgebras, quasi-finite representations, unitarizable representations, character formulae.

The first author was partially supported by NSC-grant 92-2115-M-006-016 of the R.O.C.

The second author was partially supported by the Australian Research Council.

(C)2005 American Mathematical Society Reverts to public domain 28 years from publication 
$\frac{1}{2} \mathbb{Z}$-graded module $M=\bigoplus_{j \in \frac{1}{2} \mathbb{Z}} M_{j}$ over a $\frac{1}{2} \mathbb{Z}$-graded Lie superalgebra will be called quasi-finite if all its homogeneous subspaces $M_{j}$ are finite dimensional. One of our results in the present paper is the classification of all the quasi-finite irreducible highest weight modules over $\widehat{\mathrm{gl}}_{\infty \mid \infty}, \widehat{\mathrm{C}}$ and $\widehat{\mathcal{D}}$.

It is well known that the energy of a quantum system is always bounded below, and the space of the physical states admits a positive definite contravariant Hermitian form, where the latter property is required by the probabilistic interpretation of quantum theory. Therefore, the representations of Lie superalgebras potentially useful in quantum physics are unitarizable highest weight (or lowest weight) representations. Another result of the present paper is the classification of the unitarizable quasi-finite irreducible highest weight modules over $\widehat{\mathrm{gl}}_{\infty}, \widehat{\mathrm{C}}$ and $\widehat{\mathcal{D}}$ with respect to some natural $\mathbb{C}$-conjugate linear anti-involutions of these Lie superalgebras.

We investigate the unitarizable irreducible quasi-finite highest weight modules in depth, obtaining a number of results in this direction. We first realize these irreducible representations on Fock spaces of free quantum fields. We then prove generalized Howe dualities between the infinite rank Lie superalgebras and certain classical Lie groups, establishing one-to-one correspondences between the unitarizable irreducible quasi-finite highest weight modules of the former with finite dimensional irreducible representations of the latter. Finally, we derive explicit formulae for the formal characters of the unitarizable quasi-finite irreducible highest weight modules over the Lie superalgebras $\widehat{\mathcal{C}}$ and $\widehat{\mathcal{D}}$. (The formal characters of the unitarizable irreducible modules over $\widehat{\mathrm{gl}}_{\infty \mid \infty}$ were obtained in $\underline{3}$.)

The method used here to construct the character formulae is a generalization of that developed in 3, 8, 4, by using Howe dualities [13, 14. Howe dualities for Lie superalgebras were known in the original paper of Howe 13, and were further studied in [22, 23] and [24, 25, 20, 21, 5, 6. Recent investigations on Howe dualities by ourselves with Cheng [8, 3] led to a thorough understanding of the Segal-Shale-Weil representations of Lie superalgebras. In [12] and [24, 25] Howe dualities were established respectively in the contexts of affine Kac-Moody algebras and infinite rank Lie algebras. The Howe dualities obtained in the present paper are generalizations of those studied by Wang in [24, 25].

The structure of the paper is as follows. Section 2 provides some background material on generalized partitions and unitarizable modules over Lie superalgebras. Section 3 examines central extensions of the Lie superalgebra $\left.\mathrm{gl}_{\infty}\right|_{\infty}$ of infinite matrices and its osp-type subalgebras, and gives the definitions of the Lie superalgebra $\widehat{\mathrm{gl}}_{\infty \mid \infty}$, and its subalgebras $\widehat{\mathcal{A}}, \widehat{\mathcal{C}}$ and $\widehat{\mathcal{D}}$. The remaining three sections constitute the main body of the paper. In Section 4 , we classify the quasi-finite irreducible highest weight modules over the Lie superalgebra $\left.\widehat{g l}_{\infty}\right|_{\infty}$, and its subalgebras $\widehat{\mathcal{A}}$, $\widehat{\mathcal{C}}$ and $\widehat{\mathcal{D}}$. In Section $[5$ we classify the unitarizable quasi-finite irreducible highest weight modules over these Lie superalgebras with respect to specific $*$-structures, and construct Fock space realizations of the unitarizable irreducible modules. Generalized Howe dualities between the infinite rank Lie superalgebras and classical Lie groups are also established in this section. They are used in Section 6 to derive character formulae for the unitarizable quasi-finite irreducible highest weight modules over $\widehat{\mathcal{C}}$ and $\widehat{\mathcal{D}}$. 


\section{Preliminaries}

We work over the field $\mathbb{C}$ of complex numbers throughout the paper. For any vector space $V$, we shall denote its dual space by $V^{*}$.

2.1. Shifted Frobenius notation for generalized partitions. We recall that a partition $\lambda$ of length $d=l(\lambda)$ is a non-increasing finite sequence of non-negative integers $\left(\lambda_{1}, \cdots, \lambda_{d}\right)$ and $\lambda^{\prime}$ denotes the transpose of the partition $\lambda$. Then $\lambda^{\prime}$ is a partition of length $\lambda_{1}$. The rank of a partition $\lambda$, denoted by $\operatorname{rank}(\lambda)$, is the largest integer $i$, for which $\lambda_{i} \geq i$. Note that $\operatorname{rank}(\lambda)=\operatorname{rank}\left(\lambda^{\prime}\right) \leq d$. By a generalized partition $\lambda$ of length $d=l(\lambda)$, we shall mean a non-increasing finite sequence of integers $\left(\lambda_{1}, \cdots, \lambda_{d}\right)$. Corresponding to each generalized partition $\lambda=\left(\lambda_{1}, \ldots, \lambda_{d}\right)$, we will define $\lambda^{*}:=\left(-\lambda_{d}, \ldots,-\lambda_{1}\right)$. Then $\lambda^{*}$ is also a generalized partition. In particular, if $\lambda=\left(\lambda_{1}, \ldots, \lambda_{d}\right)$ is a generalized partition of non-positive integers, then $\lambda^{*}=\left(-\lambda_{d}, \ldots,-\lambda_{1}\right)$ is a partition. In this case, we define the rank of $\lambda$ by $\operatorname{rank}(\lambda):=-\operatorname{rank}\left(\lambda^{*}\right)$. We also set $\lambda_{j}^{\prime}:=-\left(\lambda^{*}\right)_{-j}^{\prime}$ for all $j \in\left\{-1,-2, \cdots, \lambda_{d}\right\}$.

Each generalized partition $\lambda=\left(\lambda_{1}, \cdots, \lambda_{d}\right)$ of length $d$ can be uniquely expressed as $\lambda=\lambda^{+}+\lambda^{-}$, with

$$
\lambda^{+}:=\left(\max \left\{\lambda_{1}, 0\right\}, \cdots, \max \left\{\lambda_{d}, 0\right\}\right), \quad \lambda^{-}:=\left(\min \left\{\lambda_{1}, 0\right\}, \cdots, \min \left\{\lambda_{d}, 0\right\}\right) .
$$

Note that $\lambda^{+}$is a partition, while $\lambda^{-}$is a generalized partition of non-positive integers. Furthermore,

$$
\text { depth of } \lambda^{+}+\text {depth of }\left(\lambda^{-}\right)^{*} \leq d,
$$

where the depth of a partition is the number of positive integers in it.

Now we will define the shifted Frobenius notation for generalized partitions (see [19]) which will be used to describe the highest weights of unitarizable irreducible quasi-finite modules over $\widehat{\mathrm{gl}}_{\infty \mid \infty}$. Given a partition $\lambda=\left(\lambda_{1}, \ldots, \lambda_{d}\right)$ of length $d$ and $\operatorname{rank}(\lambda)=r>0$, we let $\xi_{i}:=\lambda_{i+\frac{1}{2}}-i+\frac{1}{2}$ and $\xi_{j}:=\lambda_{j}^{\prime}-j$, for $i \in \frac{1}{2}+\mathbb{Z}_{+}$with $\frac{1}{2} \leq i \leq r-\frac{1}{2}$, and $j \in \mathbb{N}$ with $1 \leq j \leq r$. We have

$$
\xi_{\frac{1}{2}}>\xi_{\frac{3}{2}}>\cdots>\xi_{r-\frac{1}{2}}>0, \quad \xi_{1}>\xi_{2}>\cdots>\xi_{r} \geq 0 .
$$

The shifted Frobenius notation for the partition $\lambda$ is given by

$$
\Lambda(\lambda):=\left(\xi_{\frac{1}{2}}, \xi_{\frac{3}{2}}, \cdots, \xi_{r-\frac{1}{2}} \mid \xi_{1}, \xi_{2}, \cdots, \xi_{r}\right) .
$$

Clearly, we have

$$
\xi_{1}+\min \left\{\xi_{\frac{1}{2}}, 1\right\} \leq d
$$

When $\lambda=(0,0, \cdots, 0)$, we set $\Lambda(\lambda):=(0,0)$. Note that (2.2) and (2.3) imply $r \leq d$.

Conversely, if two finite sequences $\xi_{\frac{1}{2}}, \xi_{\frac{3}{2}}, \cdots, \xi_{r-\frac{1}{2}}$ and $\xi_{1}, \xi_{2}, \cdots, \xi_{r}$ of nonnegative integers of length $r$ satisfy (2.2) and (2.3), we may regard them as the shifted Frobenius notation of a unique partition of length $d$, which we will denote by $F\left(\xi_{\frac{1}{2}}, \xi_{\frac{3}{2}}, \cdots, \xi_{r-\frac{1}{2}} \mid \xi_{1}, \xi_{2}, \cdots, \xi_{r}\right)$. We put $F(0,0):=(0,0, \cdots, 0)$. Thus we have a one-to-one correspondence between the set of all partitions of length $d$ and the set of all pairs of finite sequences of non-negative integers, $\xi_{\frac{1}{2}}, \xi_{\frac{3}{2}}, \cdots, \xi_{r-\frac{1}{2}}$ and $\xi_{1}, \xi_{2}, \cdots, \xi_{r}$ satisfying (2.3) and the following conditions

$$
\begin{gathered}
\xi_{\frac{1}{2}}>\xi_{\frac{3}{2}}>\cdots>\xi_{r-\frac{1}{2}} \geq 0, \quad \xi_{1}>\xi_{2}>\cdots>\xi_{r} \geq 0 \\
\xi_{r-\frac{1}{2}}=0 \text { if and only if } r=1 \text { and } \xi_{\frac{1}{2}}=\xi_{1}=0 .
\end{gathered}
$$


Similarly, given any non-zero generalized partition $\lambda=\left(\lambda_{1}, \ldots, \lambda_{d}\right)$ of nonpositive integers with $\operatorname{rank}(\lambda)=s$, the shifted Frobenius notation for the generalized partition $\lambda$ of non-positive integers is defined by

$$
\Lambda(\lambda):=\left(\xi_{s+\frac{1}{2}}, \xi_{s+\frac{3}{2}}, \cdots, \xi_{-\frac{1}{2}} \mid \xi_{s+1}, \xi_{s+2}, \cdots, \xi_{0}\right)
$$

where $\xi_{i}:=\lambda_{i-1}^{\prime}-i$ and $\xi_{j}:=\lambda_{d+\frac{1}{2}+j}-j+\frac{1}{2}$ for all $i \in\{0,-1,-2, \cdots, s+1\}$ and $j \in\left\{-\frac{1}{2},-\frac{3}{2}, \cdots, s+\frac{1}{2}\right\}$. We also define $\Lambda(0,0, \cdots, 0):=(0,0)$. Similarly, we have a one-to-one correspondence between the set of all generalized partitions of nonpositive integers of length $d$ and the set of all pairs of finite sequences of non-positive integers, $\xi_{s+\frac{1}{2}}, \xi_{s+\frac{3}{2}}, \cdots, \xi_{-\frac{1}{2}}$ and $\xi_{s+1}, \xi_{s+2}, \cdots, \xi_{0}$, satisfying the conditions

$$
\begin{aligned}
0 \geq \xi_{s+\frac{1}{2}} & >\xi_{s+\frac{3}{2}}>\cdots>\xi_{-\frac{1}{2}}, \quad 0 \geq \xi_{s+1}>\xi_{s+2}>\cdots>\xi_{0}, \\
\xi_{s+1} & =0 \text { if and only if } s=-1 \text { and } \xi_{-\frac{1}{2}}=\xi_{0}=0
\end{aligned}
$$

and

$$
-\xi_{0} \leq d
$$

For a non-zero generalized partition $\lambda$ of length $d$, the shifted Frobenius notation for $\lambda$ is defined by

$$
\Lambda(\lambda):=\left(\Lambda\left(\lambda^{-}\right) \mid \Lambda\left(\lambda^{+}\right)\right)
$$

There is a one-to-one correspondence between the set of all generalized partitions of length $d$ and the set of all quartets of finite sequences of integers, $\xi_{s+\frac{1}{2}}, \xi_{s+\frac{3}{2}}, \cdots$, $\xi_{-\frac{1}{2}} ; \xi_{s+1}, \xi_{s+2}, \cdots, \xi_{0} ; \xi_{\frac{1}{2}}, \xi_{\frac{3}{2}}, \cdots, \xi_{r-\frac{1}{2}}$ and $\xi_{1}, \xi_{2}, \cdots, \xi_{r}$ satisfying (2.4), (2.5) and

$$
\min \left\{\xi_{\frac{1}{2}}, 1\right\}+\xi_{1}-\xi_{0} \leq d
$$

We will denote by

$$
F\left(\xi_{s+\frac{1}{2}}, \xi_{s+\frac{3}{2}}, \cdots, \xi_{-\frac{1}{2}}\left|\xi_{s+1}, \xi_{s+2}, \cdots, \xi_{0}\right| \xi_{\frac{1}{2}}, \xi_{\frac{3}{2}}, \cdots, \xi_{r-\frac{1}{2}} \mid \xi_{1}, \xi_{2}, \cdots, \xi_{r}\right),
$$

the unique generalized partition corresponding to the quartet of finite sequences of integers $\xi_{s+\frac{1}{2}}, \xi_{s+\frac{3}{2}}, \cdots, \xi_{-\frac{1}{2}} ; \xi_{s+1}, \xi_{s+2}, \cdots, \xi_{0} ; \xi_{\frac{1}{2}}, \xi_{\frac{3}{2}}, \cdots, \xi_{r-\frac{1}{2}}$ and $\xi_{1}, \xi_{2}, \cdots, \xi_{r}$ satisfying (2.4), (2.5) and (2.7).

2.2. Unitarizable modules. We recall some basic facts about $*$-superalgebras and their unitarizable modules. A *-superalgebra is an associative superalgebra $A$ together with an anti-linear anti-involution $\omega: A \rightarrow A, \omega(a b)=\omega(b) \omega(a)$. A *superalgebra homomorphism $f:(A, \omega) \rightarrow\left(A^{\prime}, \omega^{\prime}\right)$ is a superalgebra homomorphism obeying $f \circ \omega=\omega^{\prime} \circ f$. Let $(A, \omega)$ be a $*$-superalgebra, and let $M$ be a $\mathbb{Z}_{2^{-}}$ graded $A$-module. A Hermitian form $\langle\cdot \mid \cdot\rangle$ on $M$ is said to be contravariant if $\left\langle a v \mid v^{\prime}\right\rangle=\left\langle v \mid \omega(a) v^{\prime}\right\rangle$, for all $a \in A, v, v^{\prime} \in M$. An $A$-module $M$ is called unitarizable if $M$ admits a positive definite contravariant Hermitian form.

Let $\mathfrak{g}$ be a Lie superalgebra together with an anti-linear anti-involution $\omega$ (i.e., $\omega$ is an anti-linear map satisfying $\omega([x, y])=[\omega(y), \omega(x)]$ for all $x, y \in \mathfrak{g}$. In this case, we also call $\omega$ a $*$-structure of $\mathfrak{g})$. Let $M$ be a $\mathfrak{g}$-module. An Hermitian form $\langle\cdot \mid \cdot\rangle$ on $M$ is said to be contravariant if $\left\langle x v \mid v^{\prime}\right\rangle=\left\langle v \mid \omega(x) v^{\prime}\right\rangle$, for all $x \in \mathfrak{g}, v, v^{\prime} \in M$. When the Hermitian form $\langle\cdot \mid \cdot\rangle$ is positive definite, we define $\|u\|:=\sqrt{\langle u \mid u\rangle}$ for all $u \in M$. A $\mathfrak{g}$-module $M$ is called unitarizable if $M$ admits a positive definite contravariant Hermitian form. The anti-linear anti-involution $\omega$ can be naturally extended to an anti-linear anti-involution, also denoted by $\omega$, on the universal enveloping algebra $\mathcal{U}(\mathfrak{g})$ of $\mathfrak{g}$, making $(\mathcal{U}(\mathfrak{g}), \omega)$ a $*$-superalgebra. Moreover, a $\mathfrak{g}$ module $M$ is unitarizable if and only if it is a unitarizable $\mathcal{U}(\mathfrak{g})$-module. 


\section{LiE SUPERALGEBRAS OF INFINITE RANK}

We present here the infinite rank Lie superalgebras to be studied in this paper. Consider the infinite-dimensional complex superspace $\mathbb{C}^{\infty} / \infty$ with a basis $\left\{e_{j} \mid j \in\right.$ $\mathbb{Z}\}$ for the even subspace, and a basis $\left\{e_{r} \mid r \in \frac{1}{2}+\mathbb{Z}\right\}$ for the odd subspace. We introduce a $\frac{1}{2} \mathbb{Z}$-gradation on $\mathbb{C}^{\infty \mid \infty}$ by setting the degree of $e_{p}$ equal to $-p$ for all $p \in \frac{1}{2} \mathbb{Z}$. For any $p, q \in \frac{1}{2} \mathbb{Z}$, let $e_{p q}$ be the endomorphism of $\mathbb{C}^{\infty} / \infty$ defined by $e_{p q}\left(e_{r}\right)=\delta_{q r} e_{p}$. Then $T$ is a homogeneous endomorphism on $\mathbb{C}^{\infty} / \infty$ of degree $p$ if and only if $T=\sum_{j \in \frac{1}{2} \mathbb{Z}} a_{j} e_{j-p, j}$, where $a_{j} \in \mathbb{C}$. Denote by $\left(M_{\infty \mid \infty}\right)_{p}$ the set of all endomorphisms of $\mathbb{C}^{\infty \mid \infty}$ of degree $p$, and let $M_{\infty \mid \infty}:=\bigoplus_{p \in \frac{1}{2} \mathbb{Z}}\left(M_{\infty \mid \infty}\right)_{p}$. Then $M_{\infty \mid \infty}$ is a $\frac{1}{2} \mathbb{Z}$-graded associative superalgebra, which also acquires a Lie superalgebra structure with the usual Lie super-bracket

$$
[A, B]:=A B-(-1)^{|A||B|} B A,
$$

where $|T|$ denotes the parity of $T \in M_{\infty}$ : $|T|=0(|T|=1)$ if the degree of $T$ is even (odd). We shall denote this Lie superalgebra by $\mathrm{gl}_{\infty \mid \infty}:=\bigoplus_{p \in \frac{1}{2} \mathbb{Z}}\left(\mathrm{gl}_{\infty \mid \infty}\right)_{p}$. Note that the subspace $\mathrm{gl}_{\infty \mid \infty}^{f}$ generated by $\left\{e_{i j} \mid i, j \in \frac{1}{2} \mathbb{Z}\right\}$ is a subalgebra of $\mathrm{gl}_{\infty \mid \infty}$. By arranging the basis elements of $\mathbb{C}^{\infty \mid \infty}$ in strictly increasing order, any endomorphism of $\mathbb{C}^{\infty \mid \infty}$ may be written as a square matrix of an infinite size with coefficients in $\mathbb{C}$. Thus

$$
\mathrm{gl}_{\infty \mid \infty}:=\left\{\left(a_{i j}\right), i, j \in \frac{1}{2} \mathbb{Z} \mid a_{i j}=0 \text { for }|j-i|>>0\right\} .
$$

Note that $\mathrm{gl}_{\infty \mid \infty}$ contains a Lie subsuperalgebra $\mathcal{A}:=\mathcal{A}^{0} \oplus \mathcal{A}^{1}$ with

$$
\mathcal{A}^{\epsilon}:=\left\{\left(a_{i j}\right) \in \mathrm{gl}_{\infty \mid \infty} \mid a_{i j}=0 \text { if } i=0 \text { or } j=0,\left|\left(a_{i j}\right)\right|=\epsilon\right\}, \quad \epsilon=0,1 .
$$

Furthermore, $\mathcal{A}^{f}:=\mathcal{A} \cap \mathrm{gl}_{\infty \mid \infty}^{f}$ is a Lie subsuperalgebra of $\mathrm{gl}_{\infty \mid \infty}^{f}$.

Introduce a non-degenerate skew-supersymmetric bilinear form $(\cdot \mid \cdot)$ on $\mathbb{C}^{\infty / \infty}$ defined by

$$
\begin{aligned}
& \left(e_{i} \mid e_{j}\right)=-\left(e_{j} \mid e_{i}\right)=\operatorname{sgn}(\mathrm{i}) \delta_{i,-j}, \quad i, j \in \mathbb{Z}^{*} ; \\
& \left(e_{r} \mid e_{s}\right)=\left(e_{s} \mid e_{r}\right)=\delta_{r,-s}, \quad r, s \in \frac{1}{2}+\mathbb{Z} ; \\
& \left(e_{i} \mid e_{r}\right)=\left(e_{r} \mid e_{i}\right)=0, \quad i \in \mathbb{Z}^{*}, r \in \frac{1}{2}+\mathbb{Z} ;
\end{aligned}
$$

where $\operatorname{sgn}(\mathrm{i})=+1$ if $i \in \frac{1}{2} \mathbb{N}$ and $\operatorname{sgn}(\mathrm{i})=-1$ if $i \in-\frac{1}{2} \mathbb{N}$. We define the Lie superalgebra $\mathcal{C}:=\mathcal{C}^{0} \oplus \mathcal{C}^{1}$ to be the $\frac{1}{2} \mathbb{Z}$-graded Lie subsuperalgebra of $\mathcal{A}$ preserving this form, i.e.,

$$
\mathcal{C}^{\epsilon}:=\left\{A \in \mathcal{A}^{\epsilon} \mid(A v \mid w)=-(-1)^{\epsilon|v|}(v \mid A w)\right\}, \quad \epsilon=0,1 .
$$

Here and further $|v|$ denotes the parity of $v \in \mathbb{C}^{\infty} \mid \infty$, namely, $|v|=0$ (respectively 1 ) if $v$ belongs to the even (respectively odd) homogeneous subspace of $\mathbb{C}^{\infty / \infty}$. Then $\mathrm{C}$ is a Lie superalgebra of type $S P O$. It is easy to see that the subsuperalgebra 
$\mathcal{C}^{f}:=\mathcal{C} \cap \mathrm{gl}_{\infty \mid \infty}^{f}$ is spanned by the following elements $\left(i, j \in \mathbb{Z}^{*}, r, s \in \frac{1}{2}+\mathbb{Z}\right)$ :

$$
\begin{array}{ll}
\tilde{e}_{i, j}:=-\tilde{e}_{-j,-i}:=e_{i, j}-e_{-j,-i}, & i j>0 ; \\
\tilde{e}_{i, j}:=\tilde{e}_{-j,-i}:=e_{i, j}+e_{-j,-i}, & i j<0 ; \\
\tilde{e}_{r, s}:=-\tilde{e}_{-s,-r}:=e_{r, s}-e_{-s,-r} ; & \\
\tilde{e}_{i, r}:=\tilde{e}_{-r,-i}:=e_{i, r}+e_{-r,-i}, & i>0 ; \\
\tilde{e}_{i, r}:=-\tilde{e}_{-r,-i}:=e_{i, r}-e_{-r,-i}, & i<0 .
\end{array}
$$

Let $(\cdot \mid \cdot)$ be the non-degenerate supersymmetric bilinear form on $\mathbb{C}^{\infty} \mid \infty$ defined by

$$
\begin{array}{lr}
\left(e_{i} \mid e_{j}\right)=\left(e_{j} \mid e_{i}\right)=\delta_{i,-j}, & i, j \in \mathbb{Z}^{*} \\
\left(e_{r} \mid e_{s}\right)=-\left(e_{s} \mid e_{r}\right)=\operatorname{sgn}(\mathrm{r}) \delta_{r,-s}, & r, s \in \frac{1}{2}+\mathbb{Z} \\
\left(e_{i} \mid e_{r}\right)=\left(e_{r} \mid e_{i}\right)=0, & i \in \mathbb{Z}^{*}, r \in \frac{1}{2}+\mathbb{Z}
\end{array}
$$

We define the Lie superalgebra $\mathcal{D}=\mathcal{D}^{0} \oplus \mathcal{D}^{1}$ to be the subsuperalgebra of $\mathcal{A}$ preserving this form, i.e.,

$$
\mathcal{D}^{\epsilon}=\left\{A \in \mathcal{A}_{\epsilon} \mid(A v \mid w)=-(-1)^{\epsilon|v|}(v \mid A w)\right\}, \quad \epsilon=0,1 .
$$

This is a Lie superalgebra of type $O S P$. It is easy to see that the subsuperalgebra $\mathcal{D}^{f}:=\mathcal{D} \cap \mathrm{gl}_{\infty \mid \infty}^{f}$ is spanned by the following elements $\left(i, j \in \mathbb{Z}^{*}, r, s \in \frac{1}{2}+\mathbb{Z}\right)$ :

$$
\begin{array}{lrr}
\tilde{e}_{i, j}:=-\tilde{e}_{-j,-i}:=e_{i, j}-e_{-j,-i} ; & \\
\tilde{e}_{r, s}:=-\tilde{e}_{-s,-r}:=e_{r, s}-e_{-s,-r}, & r s>0 ; \\
\tilde{e}_{r, s}:=\tilde{e}_{-s,-r}:=e_{r, s}+e_{-s,-r}, & r s<0 ; \\
\tilde{e}_{i, r}:=\tilde{e}_{-r,-i}:=e_{i, r}+e_{-r,-i}, & r>0 ; \\
\tilde{e}_{i, r}:=-\tilde{e}_{-r,-i}:=e_{i, r}-e_{-r,-i}, & r<0 .
\end{array}
$$

Remark 3.1. Note that $\mathcal{C}^{0} \cap \mathcal{C}^{f}$ and $\mathcal{D}^{0} \cap \mathcal{D}^{f}$ are a direct sum of an infinite dimensional symplectic Lie algebra and an infinite dimensional orthogonal Lie algebra.

For $\mathfrak{g}$ being $\mathrm{gl}_{\infty \mid \infty}, \mathcal{A}$, $\mathcal{C}$ or $\mathcal{D}$, we let $\hat{\mathfrak{g}}$ ( $\hat{\mathfrak{g}}^{f}$, respectively) denote the central extension of $\mathfrak{g}\left(\mathfrak{g}^{f}\right.$, respectively) by an even central element $C$ associated with the following two-cocycle:

$$
\alpha(A, B):=\operatorname{Str}([J, A] B), \quad A, B \in \mathfrak{g}\left(\mathfrak{g}^{f}, \text { respectively }\right),
$$

where $J=\sum_{r \leq 0} e_{r r}$ and Str stands for the supertrace defined for a matrix $D=$ $\left(d_{i j}\right) \in \mathrm{gl}_{\infty \mid \infty}$ by $\operatorname{Str}(D)=\sum_{r \in \frac{1}{2} \mathbb{Z}}(-1)^{2 r} d_{r r}$ provided that the infinite sum is convergent. By setting the degree of $C$ equal to 0 , the Lie superalgebras $\hat{\mathfrak{g}}$ and $\hat{\mathfrak{g}}^{f}$ acquire a $\frac{1}{2} \mathbb{Z}$-gradation from that of $\mathfrak{g}$.

\section{QuASI-FINITE MODUleS}

In this section we give a complete classification of all the quasi-finite irreducible highest weight modules over the Lie superalgebras discussed in Section 3 , 
4.1. Quasi-finite modules. Let $\mathfrak{g}=\bigoplus_{j \in \frac{1}{2} \mathbb{Z}} \mathfrak{g}_{j}$ (possibly $\operatorname{dim} \mathfrak{g}_{j}=\infty$ ) be a $\frac{1}{2} \mathbb{Z}$ graded Lie superalgebra, with the even subspace $\bigoplus_{j \in \mathbb{Z}} \mathfrak{g}_{j}$, and odd subspace $\bigoplus_{j \in \frac{1}{2}+\mathbb{Z}} \mathfrak{g}_{j}$. We assume that $\mathfrak{g}_{0}$ is abelian. We have the triangular decomposition

$$
\mathfrak{g}=\mathfrak{g}_{-} \oplus \mathfrak{g}_{0} \oplus \mathfrak{g}_{+}, \quad \text { with } \mathfrak{g}_{ \pm}=\bigoplus_{\mathrm{r}>0} \mathfrak{g}_{ \pm \mathrm{r}} .
$$

Given a graded $\mathfrak{g}$-module $M=\bigoplus_{j \in \frac{1}{2} \mathbb{Z}} M_{j}$, we call a vector $v \in M$ homogeneous of degree $j$ if $v \in M_{j}$ for some $j \in \frac{1}{2} \mathbb{Z}$. Following the terminology of Kac and Radul 17, we shall call $M$ quasi-finite if $\operatorname{dim} M_{j}<\infty$ for all $j \in \frac{1}{2} \mathbb{Z}$.

A $\mathfrak{g}$-module $M$ is called a highest weight module with highest weight $\xi \in \mathfrak{g}_{0}^{*}$ if there is a non-zero vector $v_{\xi} \in M$ satisfying the following conditions:

(i) $h v_{\xi}=\xi(h) v_{\xi}$, for all $h \in \mathfrak{g}_{0}$,

(ii) $\mathfrak{g}_{+} v_{\xi}=0$,

(iii) $\mathcal{U}\left(\mathfrak{g}_{-}\right) v_{\xi}=M$.

Then $v_{\xi}$ is called a highest weight vector of $M$. Note that the module $M$ acquires a natural $\frac{1}{2} \mathbb{Z}$-gradation by assigning a degree zero to $v_{\xi}$. More precisely, we have $M=\bigoplus_{r \in \frac{1}{2} \mathbb{Z}_{+}} M_{-r}$ and $M_{0}=\mathbb{C} v_{\xi}$. A homogeneous non-zero vector $v \in M$ is said to be singular if $\mathfrak{g}_{+} v=0$. Note that a highest weight module is irreducible if and only if the space of singular vectors is 1-dimensional. We denote by $L(\mathfrak{g}, \xi)$ the irreducible highest weight module with highest weight $\xi \in \mathfrak{g}_{0}^{*}$.

We recall the following criterion for quasi-finite highest weight modules.

Proposition 4.1 ([7]). Let $\mathfrak{g}=\bigoplus_{j \in \frac{1}{2} \mathbb{Z}} \mathfrak{g}_{j}$ be a $\frac{1}{2} \mathbb{Z}$-graded Lie superalgebra such that $\mathfrak{g}_{0}$ is abelian. Let $M=\bigoplus_{j \in \frac{1}{2} \mathbb{Z}_{+}} M_{-j}$ be a highest weight $\mathfrak{g}$-module with highest weight $\xi \in \mathfrak{g}_{0}^{*}$. For any non-zero highest weight vector $v_{\xi}$ in $M$, the subspace $\mathfrak{g}_{j} v_{\xi}$ is finite-dimensional for all $j$ if and only if $M$ is quasi-finite.

Let $M$ be a $\mathfrak{g}$-module. For any $\lambda \in \mathfrak{g}_{0}^{*}$, set

$$
M_{\lambda}=\left\{v \in M \mid h v=\lambda(h) v, \text { for all } h \in \mathfrak{g}_{0}\right\} .
$$

When $M_{\lambda} \neq 0, \lambda$ is called a weight of $M$, and $M_{\lambda}$ is called the weight space of weight $\lambda$. We let $P(M)$ denote the set of all weights of $M$. A graded $\mathfrak{g}$-module $M=\bigoplus_{j \in \frac{1}{2} \mathbb{Z}} M_{j}$ is called $\mathfrak{g}_{0}$-diagonalizable if every $M_{\lambda}$ is finite dimensional, and $M_{j}=\bigoplus_{\lambda \in P(M)}\left(M_{\lambda} \cap M_{j}\right)$, for all $j \in \frac{1}{2} \mathbb{Z}$.

For any $\lambda \in \mathfrak{g}_{0}^{*}$, we also set

$$
\mathfrak{g}_{\lambda}=\left\{x \in \mathfrak{g} \mid[h, x]=\lambda(h) x, \text { for all } h \in \mathfrak{g}_{0}\right\} .
$$

As all the infinite rank Lie superalgebras in Section 3 are $\frac{1}{2} \mathbb{Z}$-graded, the representation theoretical notions discussed above are all valid for them.

4.2. Quasi-finite modules over $\widehat{\mathrm{gl}}_{\infty \mid \infty}, \widehat{\mathcal{A}}, \widehat{\mathcal{C}}$ and $\widehat{\mathcal{D}}$. In this subsection, $\hat{\mathfrak{g}}$ will stand for any of the Lie superalgebras $\widehat{g l}_{\infty \mid \infty}, \widehat{\mathcal{A}}, \widehat{\mathcal{C}}$ and $\widehat{\mathcal{D}}$. For any $k \in \frac{1}{2} \mathbb{Z}$ and $N \in \frac{1}{2} \mathbb{Z}_{+}$, we let

$$
\left(\widehat{\mathrm{gl}}_{\infty \mid \infty}\right)_{k, N}:=\left\{x \in \widehat{\mathrm{gl}}_{\infty \mid \infty} \mid x=\sum_{j \in \frac{1}{2} \mathbb{Z},|j| \geq N} a_{j} e_{j-k, j}, a_{j} \in \mathbb{C}\right\} .
$$

For $\hat{\mathfrak{g}}=\widehat{\mathcal{A}}, \widehat{\mathcal{C}}$ or $\widehat{\mathcal{D}}$ and for any $k \in \frac{1}{2} \mathbb{Z}$ and $N \in \frac{1}{2} \mathbb{Z}_{+}$, we let $\hat{\mathfrak{g}}_{k, N}:=\hat{\mathfrak{g}} \cap\left(\widehat{\mathrm{gl}}{ }_{\infty}\right)_{k, N}$.

The following lemma can be confirmed by a straightforward computation. 
Lemma 4.1. Given any fixed positive integer or half integer $N$, we have

$$
\left[\hat{\mathfrak{g}}_{p}, \hat{\mathfrak{g}}_{-k, k+N}\right] \subset \hat{\mathfrak{g}}_{-(k-p),(k-p)+N},
$$

for all $k, p \in \frac{1}{2} \mathbb{Z}_{+}$with $p \leq k$.

The following result will be important for the classification of quasi-finite modules.

Proposition 4.2. Let $M=\bigoplus_{j \in \frac{1}{2} \mathbb{Z}_{+}} M_{-j}$ be a highest weight $\hat{\mathfrak{g}}$-module and $v_{0}$ a non-zero highest weight vector. If $\hat{\mathfrak{g}}_{-r} v_{0}$ is finite dimensional for a fixed number $r \in \frac{1}{2} \mathbb{N}$, then for every $p \in \frac{1}{2} \mathbb{Z}_{+}$with $p<r$, there exists $N \in \mathbb{N}$ such that

$$
\hat{\mathfrak{g}}_{-p, N} v_{0}=0 \text {. }
$$

In particular, $\hat{\mathfrak{g}}_{-p} v_{0}$ is finite dimensional for all $p \leq r$.

Proof. We will only prove the proposition in the case $\hat{\mathfrak{g}}=\left.\widehat{\mathrm{gl}}_{\infty}\right|_{\infty}$, as the other cases are all very much the same. Fixing a transcendental real number $\pi$, we let $w_{i}=\sum_{j \in \frac{1}{2} \mathbb{Z}} \pi^{2 i j} e_{j+r, j}$, for each $i \in \mathbb{N}$, which belong to $\left(\widehat{\mathrm{gl}}_{\infty \mid \infty}\right)_{-r}$. For any $x=\sum_{l=1}^{k} \alpha_{i_{l}} w_{i_{l}}$, where $\alpha_{i_{1}}, \alpha_{i_{2}}, \cdots, \alpha_{i_{k}}$ are non-zero complex numbers with $i_{1}<$ $i_{2}<\cdots<i_{k}$, we can rewrite it as $x=\sum_{j \in \frac{1}{2} \mathbb{Z}} \beta_{j} e_{j+r, j}$. Then by using the Vandermonde determinant it is easy to see that $\beta_{j}$ are non-zero except for finitely many $j$. Thus there always exists some positive integer $N$ with $N>r$ such that $\beta_{j} \neq 0$ for all $j$ with $|j| \geq N$. Since $\left(\widehat{\mathrm{gl}}_{\infty \mid \infty}\right)_{-r} v_{0}$ is finite dimensional, we can always find non-zero complex numbers $\alpha_{i_{1}}, \alpha_{i_{2}}, \cdots, \alpha_{i_{k}}$ so that $x=\sum_{l=1}^{k} \alpha_{i_{l}} w_{i_{l}}$ satisfies $x v_{0}=0$. We fix such an $x$.

We shall prove the proposition by contradiction. Assume that there exists $p \in$ $\frac{1}{2} \mathbb{Z}_{+}$with $p<r$ such that $\left(\widehat{\mathrm{gl}}_{\infty \mid \infty}\right)_{-p, q} v_{0} \neq 0$, for all $q \in \mathbb{N}$. Then we can find $y:=$ $\sum_{|j| \geq N} a_{j} e_{j+p, j} \in\left(\widehat{\mathrm{gl}}_{\infty \mid \infty}\right)_{-p, N}$ such that $y v_{0} \neq 0$. We claim that corresponding to each such $y$, there exits a $u=\sum_{j \in \frac{1}{2} \mathbb{Z}} b_{j} e_{j+p, j+r} \in\left(\widehat{\mathrm{gl}}_{\infty \mid \infty}\right)_{r-p}$ such that

$$
[u, x]=y .
$$

Indeed if we choose an element $u$ with the coefficients $b_{j},-N-2 r<j<N+r$, given by

$$
b_{j}:= \begin{cases}0, & \text { if } \quad-N-r<j<N ; \\ \frac{a_{j}}{\beta_{j}}, & \text { if } \quad N \leq j<N+r ; \\ \frac{-(-1)^{4 r(r-p)} a_{j+r}}{\beta_{j+p}}, & \text { if } \quad-N-2 r<j \leq-N-r ;\end{cases}
$$

and the $b_{j}$ for $j \leq-N-2 r$ or $j \geq N+r$ given recursively by

$$
b_{j}:=\left\{\begin{array}{lll}
\frac{a_{j}+(-1)^{4 r(r-p)} \beta_{j+p-r} b_{j-r}}{\beta_{j}}, & \text { if } \quad j \geq N+r ; \\
\frac{(-1)^{4 r(r-p)}\left(b_{j+r} \beta_{j+r}-a_{j+r}\right)}{\beta_{j+p}}, & \text { if } \quad j \leq-N-2 r,
\end{array}\right.
$$

then (4.1) holds true as can be shown by a direct computation. However, equation (4.1) leads to the obvious contradiction $y v_{0}=[u, x] v_{0}=0$. This completes the proof.

The following theorem is an obvious consequence of Proposition 4.1 and Proposition 4.2 
Theorem 4.1. Let $M=\bigoplus_{j \in \frac{1}{2} \mathbb{Z}_{+}} M_{-j}$ be a highest weight $\hat{\mathfrak{g}}$-module and $v_{0}$ a highest weight vector. Then $M$ is quasi-finite if and only if for every $r \in \frac{1}{2} \mathbb{Z}_{+}$, there exists $N \in \mathbb{N}$ such that

$$
\hat{\mathfrak{g}}_{-r, N} v_{0}=0
$$

In this case, $M=\mathcal{U}\left(\hat{\mathfrak{g}}^{f}\right) v_{0}$ and hence is $\hat{\mathfrak{g}}_{0}$-diagonalizable.

Let $I_{\widehat{\mathrm{gl}}}=\frac{1}{2} \mathbb{Z} ; I_{\widehat{\mathcal{A}}}=\frac{1}{2} \mathbb{Z}^{*}$, where $\mathbb{Z}^{*}=\mathbb{Z} \backslash\{0\} ;$ and $I_{\hat{\mathfrak{g}}}=\frac{1}{2} \mathbb{N}$ for $\hat{\mathfrak{g}}=\widehat{\mathcal{C}}, \widehat{\mathcal{D}}$. Denote by $\Lambda_{0}, \omega_{s}, s \in I_{\hat{\mathfrak{g}}}$ the fundamental weights of $\hat{\mathfrak{g}}$, that is, $\Lambda_{0}, \omega_{s} \in \hat{\mathfrak{g}}_{0}^{*}$ defined by

$$
\begin{array}{llrl}
\omega_{s}\left(\sum_{r \in I_{\hat{\mathfrak{g}}}} a_{r} e_{r r}+d C\right) & =a_{s}, & \Lambda_{0}\left(\sum_{r \in I_{\hat{\mathfrak{g}}}} a_{r} e_{r r}+d C\right)=d, & \text { if } \hat{\mathfrak{g}}=\left.\widehat{\mathrm{gl}}\right|_{\infty}, \widehat{\mathcal{A}}, \\
\omega_{s}\left(\sum_{r \in I_{\hat{\mathfrak{g}}}} a_{r} \tilde{e}_{r r}+d C\right)=a_{s}, & \Lambda_{0}\left(\sum_{r \in I_{\hat{\mathfrak{g}}}} a_{r} \tilde{e}_{r r}+d C\right)=d, & \text { if } \hat{\mathfrak{g}}=\widehat{\mathcal{C}}, \widehat{\mathcal{D}} .
\end{array}
$$

Theorem 4.2. Let $M$ be an irreducible highest weight $\hat{\mathfrak{g}}$-module with highest weight $\xi$ and a nonzero highest weight vector $v_{\xi}$. Then the following conditions are equivalent:

(i) $M$ is quasi-finite,

(ii) $\hat{\mathfrak{g}}_{-\frac{1}{2}} v_{\xi}$ is finite dimensional,

(iii) there exists $N \in \mathbb{N}$ such that

$$
\xi=\sum_{|j| \leq N, j \in I_{\hat{\mathfrak{g}}}} \xi_{j} \omega_{j}+d \Lambda_{0}, \quad \text { where } \xi_{j}, d \in \mathbb{C} .
$$

Proof. The proofs for all the cases are very similar, thus we shall consider only $\widehat{\mathrm{gl}}_{\infty \mid \infty}$. It clearly follows from Proposition 4.2 that (i) implies (ii), and (ii) implies (iii). Now we show that (iii) implies (i). Assume that $\xi=\sum_{|j| \leq N_{0}, j \in \frac{1}{2} \mathbb{Z}} \xi_{j} \omega_{j}+d \Lambda_{0}$. Then by Theorem 4.1, it is sufficient to show that for all $r \in \frac{1}{2} \mathbb{Z}_{+}$, there exists $N \in \mathbb{N}$ such that $\left(\widehat{\mathrm{gl}}_{\infty \mid \infty}\right)_{-r, N} v_{\xi}=0$. We shall prove it by induction. It is obviously true for $r=0$ and we assume that it is also true for all $p \in \frac{1}{2} \mathbb{N}$ with $0 \leq p<r$. Choose $N_{p} \in \mathbb{N}$ such that $\left(\left.\widehat{\mathrm{gl}}_{\infty}\right|_{\infty}\right)_{-p, N_{p}} v_{\xi}=0$. Let $N=$ $\max \left\{N_{0}+1, N_{1 / 2}, \cdots, N_{r-1 / 2}\right\}$. For all $p \in \frac{1}{2} \mathbb{N}$ with $p>r$, it is clear that $\left(\widehat{\mathrm{gl}}_{\infty \mid \infty}\right)_{p}\left(\widehat{\mathrm{gl}}_{\infty \mid \infty}\right)_{-r, r+N} v_{\xi} \subseteq\left(\widehat{\mathrm{gl}}_{\infty \mid \infty}\right)_{p-r} v_{\xi}=0$. By Lemma 4.1, we also have $\left(\widehat{\mathrm{gl}}_{\infty \mid \infty}\right)_{p}\left(\widehat{\mathrm{gl}}_{\infty \mid \infty}\right)_{-r, r+N} v_{\xi} \subseteq\left(\widehat{\mathrm{gl}}_{\infty \mid \infty}\right)_{-(r-p),(r-p)+N} v_{\xi}=0$, for all $p \in \frac{1}{2} \mathbb{N}$ with $0<$ $p \leq r$. Thus $\left(\widehat{\mathrm{gl}}_{\infty \mid \infty}\right)_{+}\left(\widehat{\mathrm{gl}}_{\infty \mid \infty}\right)_{-r, r+N} v_{\xi}=0$. Using Lemma 4.1 again, we also have $\left.\left(\widehat{\mathrm{gl}}_{\infty \mid \infty}\right)_{0}\left(\widehat{\mathrm{gl}}_{\infty \mid \infty}\right)_{-r, r+N} v_{\xi} \subseteq\left(\widehat{\mathrm{gl}}_{\infty}\right)_{\infty}\right)_{-r, r+N} v_{\xi}$, so $\mathcal{U}\left(\left(\widehat{\mathrm{gl}}_{\infty \mid \infty}\right)_{-}\right)\left(\widehat{\mathrm{gl}}_{\infty \mid \infty}\right)_{-r, r+N} v_{\xi}$ is a proper submodule of $M$. Thus we have $\left(\widehat{\mathrm{gl}}_{\infty \mid \infty}\right)_{-r, r+N} v_{\xi}=0$ since $M$ is irreducible.

Remark 4.1. Let $\widehat{\mathrm{gl}}_{\infty}$ be the the $\mathbb{Z}$-graded Lie subalgebra

$$
\widehat{\mathrm{gl}}_{\infty}:=\left\{\left(a_{i j}\right) \in \widehat{\mathrm{gl}}_{\infty \mid \infty} \mid a_{i j}=0 \text { for } i=\frac{1}{2} \text { or } j=\frac{1}{2}\right\}+\mathbb{C} C
$$

of $\widehat{\mathrm{gl}}_{\infty \mid \infty}$, which inherits a natural $\mathbb{Z}$-gradation from $\widehat{\mathrm{gl}}_{\infty \mid \infty}$. Then all the results in this section can be restricted to the non-super case, leading to descriptions and classifications of the irreducible quasi-finite highest weight modules over $\widehat{\mathrm{gl}}_{\infty}$ and its $\mathbb{Z}$-graded subalgebras. For example, we have the following theorem. 
Theorem 4.3. Let $M$ be an irreducible highest weight $\widehat{\mathrm{gl}}_{\infty}$-module and $v_{\xi}$ a nonzero highest weight vector. Then the following are equivalent:

(i) $M$ is quasi-finite,

(ii) $\left(\widehat{\mathrm{gl}}_{\infty}\right)_{-1} v_{\xi}$ is finite dimensional,

(iii) there exists $N \in \mathbb{N}$ such that $\xi=\sum_{|j| \leq N} \xi_{j} \omega_{j}+d \Lambda_{0}$, where $\xi_{j}, d \in \mathbb{C}$,

where $\Lambda_{0}, \omega_{i}, i \in \mathbb{Z}$ are the fundamental weights of $\widehat{\mathrm{gl}}_{\infty}$.

\section{UNITARIZABLE REPRESENTATIONS AND THEIR FREE FIELD REALIZATIONS}

In this section we study in depth the quasi-finite irreducible highest weight modules over $\widehat{g l}_{\infty \mid \infty}, \widehat{\mathcal{A}}, \widehat{\mathrm{C}}$ and $\widehat{\mathcal{D}}$, which are unitarizable with respect to natural choices of *-structures for these Lie superalgebras. The main results obtained are the following: (1) the classification of the unitarizable quasi-finite irreducible highest weight modules over the Lie superalgebras $\widehat{\mathrm{gl}}_{\infty \mid \infty}, \widehat{\mathcal{A}}, \widehat{\mathcal{C}}$ and $\widehat{\mathcal{D}} ;(2)$ realizations of these irreducible modules on Fock spaces; and (3) generalized Howe dualities between these infinite rank Lie superalgebras and classical Lie algebras. The generalized Howe dualities will provide the principal tool for constructing character formulae for the unitarizable quasi-finite irreducible highest weight modules in the next section.

5.1. Free field realizations of $\widehat{\mathrm{gl}}_{\infty \mid \infty}$ and $\left(\widehat{\mathrm{gl}}_{\infty \mid \infty}, g l_{d}\right)$-duality. Let $g l_{d}$ denote the Lie algebra of all complex $d \times d$ matrices. Let $\left\{e^{1}, \ldots, e^{d}\right\}$ be the standard basis for $\mathbb{C}^{d}$. Denote by $E_{i j}$ the matrix unit with the entry at the $i$-th row and $j$-th column being 1 and all other entries being 0 . Then $\mathfrak{h}_{d}:=\sum_{i=1}^{d} \mathbb{C} E_{i i}$ is a Cartan subalgebra, while $\mathfrak{b}_{d}:=\sum_{1 \leq i \leq j \leq d} \mathbb{C} E_{i j}$ is the standard Borel subalgebra containing $\mathfrak{h}_{d}$. We regard a finite sequence $\bar{\lambda}=\left(\lambda_{1}, \cdots, \lambda_{d}\right)$ of complex numbers as an element of the dual vector space $\mathfrak{h}_{d}^{*}$ of $\mathfrak{h}_{d}$ defined by $\lambda\left(E_{i i}\right)=\lambda_{i}$, for $i=1, \cdots, d$. Denote by $V_{d}^{\lambda}$ the irreducible $g l_{d}$-module with highest weight $\lambda$ relative to the standard Borel subalgebra $\mathfrak{b}_{d}$.

Consider $d$ pairs of free fermionic fields $\psi^{ \pm, i}(z)$ and $d$ pairs of free symplectic bosonic fields $\gamma^{ \pm, i}(z), i=1, \cdots, d$, with the following mode expansions:

$$
\begin{array}{rlrl}
\psi^{+, i}(z) & =\sum_{n \in \mathbb{Z}} \psi_{n}^{+, i} z^{-n-1}, & \psi^{-, i}(z) & =\sum_{n \in \mathbb{Z}} \psi_{n}^{-, i} z^{-n}, \\
\gamma^{+, i}(z) & =\sum_{r \in \frac{1}{2}+\mathbb{Z}} \gamma_{r}^{+, i} z^{-r-1 / 2}, & \gamma^{-, i}(z)=\sum_{r \in \frac{1}{2}+\mathbb{Z}} \gamma_{r}^{-, i} z^{-r-1 / 2},
\end{array}
$$

where the operators $\psi_{n}^{+, i}$ and $\gamma_{r}^{+, i}$ satisfy the usual (anti-)commutation relations with the non-trivial ones being given by

$$
\psi_{m}^{+, i} \psi_{n}^{-, j}+\psi_{n}^{-, j} \psi_{m}^{+, i}=\delta_{i j} \delta_{m+n, 0}, \quad \gamma_{r}^{+, i} \gamma_{s}^{-, j}-\gamma_{s}^{-, j} \gamma_{r}^{+, i}=\delta_{i j} \delta_{r+s, 0} .
$$

Denote by $\mathscr{A}^{d}$ the associative superalgebra generated by these operators. We shall take the operators $\psi_{n}^{+, i}, \psi_{m}^{-, i}, \gamma_{r}^{ \pm, i}$, with $i=1,2, \cdots, d, n \geq 0, m>0, r>0$, as annihilation operators, and the rest as creation operators. Let $\mathfrak{F}^{d}$ be the Fock space of $\mathscr{A}^{d}$ generated by the vacuum vector $|0\rangle$. 
There is an action of $\widehat{\mathrm{gl}}_{\infty \mid \infty}$ with central charge $d$ on the Fock space $\mathfrak{F}^{d}$ defined as follows:

$$
\begin{array}{ll}
e_{i j}=\sum_{p=1}^{d}: \psi_{-i}^{+, p} \psi_{j}^{-, p}:, & e_{r s}=-\sum_{p=1}^{d}: \gamma_{-r}^{+, p} \gamma_{s}^{-, p}:, \\
e_{i s}=\sum_{p=1}^{d}: \psi_{-i}^{+, p} \gamma_{s}^{-, p}:, & e_{r j}=-\sum_{p=1}^{d}: \gamma_{-r}^{+, p} \psi_{j}^{-, p}:,
\end{array}
$$

where $i, j \in \mathbb{Z}$ and $r, s \in \frac{1}{2}+\mathbb{Z}$. Hereafter :: denotes normal ordering with respect to the vacuum $|0\rangle$. There also exists an action of $g l_{d}$ on $\mathfrak{F}^{d}$, which is given by the formula

$$
E_{i j}=\sum_{n \in \mathbb{Z}}: \psi_{-n}^{+, i} \psi_{n}^{-, j}:-\sum_{r \in 1 / 2+\mathbb{Z}}: \gamma_{-r}^{+, i} \gamma_{r}^{-, j}:
$$

For a generalized partition $\lambda=\left(\lambda_{1}, \lambda_{2}, \cdots, \lambda_{d}\right)$ of length $d$, we have the shifted Frobenius notation (see Section 2.1)

$$
\Lambda(\lambda)=\left(\xi_{s+\frac{1}{2}}, \xi_{s+\frac{3}{2}}, \cdots, \xi_{-\frac{1}{2}}\left|\xi_{s+1}, \xi_{s+2}, \cdots, \xi_{0}\right| \xi_{\frac{1}{2}}, \xi_{\frac{3}{2}}, \cdots, \xi_{r-\frac{1}{2}} \mid \xi_{1}, \xi_{2}, \cdots, \xi_{r}\right) .
$$

We identify $\Lambda(\lambda)$ with an element of the dual space $\left(\widehat{\mathrm{gl}}_{\infty \mid \infty}\right)_{0}^{*}$ of $\left(\left.\widehat{\mathrm{gl}}_{\infty}\right|_{\infty}\right)_{0}$ defined by

$$
\Lambda(\lambda):=\sum_{s+\frac{1}{2} \leq j \leq r, j \in \frac{1}{2} \mathbb{Z}} \xi_{j} \omega_{j}+d \Lambda_{0} .
$$

The following result is the $\left(\widehat{\mathrm{gl}}_{\infty \mid \infty}, g l_{d}\right)$-duality of [7].

Theorem 5.1 ([7]). The Lie superalgebra $\widehat{\mathrm{gl}}_{\infty \mid \infty}$ and $\mathrm{gl}_{d}$ form a dual pair on $\mathfrak{F}^{d}$ in the sense of Howe. Furthermore, we have the following (multiplicity-free) decomposition of $\mathfrak{F}^{d}$ with respect to their joint action

$$
\mathfrak{F}^{d} \cong \sum_{\lambda} L\left(\widehat{\mathrm{gl}}_{\infty \mid \infty}, \Lambda(\lambda)\right) \otimes V_{d}^{\lambda},
$$

where $\lambda$ runs over all generalized partitions of length $d$.

5.2. Free field realizations of $\widehat{\mathcal{A}}$ and $\left(\widehat{\mathcal{A}}, g l_{d}\right)$-duality. This time we take the following free fermions $\widetilde{\psi}^{ \pm, i}(z)$ and free symplectic bosons $\gamma^{ \pm, i}(z)(i=1, \cdots, d)$

$$
\begin{aligned}
\widetilde{\psi}^{+, i}(z) & =\sum_{n \in \mathbb{Z}^{*}} \psi_{n}^{+, i} z^{-n-1}, & \widetilde{\psi}^{-, i}(z) & =\sum_{n \in \mathbb{Z}^{*}} \psi_{n}^{-, i} z^{-n}, \\
\gamma^{+, i}(z) & =\sum_{r \in \frac{1}{2}+\mathbb{Z}} \gamma_{r}^{+, i} z^{-r-1 / 2}, & \gamma^{-, i}(z) & =\sum_{r \in \frac{1}{2}+\mathbb{Z}} \gamma_{r}^{-, i} z^{-r-1 / 2}
\end{aligned}
$$

where the non-trivial anti-commutation relations are still of the form (5.1). Let $\mathfrak{F}_{0}^{d}$ denote the corresponding Fock space generated by the vacuum vector $|0\rangle$ with $\psi_{m}^{ \pm, i}|0\rangle=\gamma_{r}^{ \pm, i}|0\rangle=0$, for $i=1,2, \cdots, d, m>0$ and $r>0$.

Then $\widehat{\mathcal{A}}$ with central charge $d$ is realized on $\mathfrak{F}_{0}^{d}$ by $\left(i, j \in \mathbb{Z}^{*}\right.$ and $\left.r, s \in \frac{1}{2}+\mathbb{Z}\right)$

$$
\begin{array}{ll}
e_{i j}=\sum_{p=1}^{d}: \psi_{-i}^{+, p} \psi_{j}^{-, p}:, & e_{r s}=-\sum_{p=1}^{d}: \gamma_{-r}^{+, p} \gamma_{s}^{-, p}:, \\
e_{i s}=\sum_{p=1}^{d}: \psi_{-i}^{+, p} \gamma_{s}^{-, p}:, & e_{r j}=-\sum_{p=1}^{d}: \gamma_{-r}^{+, p} \psi_{j}^{-, p}:,
\end{array}
$$


and an action of $g l_{d}$ on $\mathfrak{F}_{0}^{d}$ is given by the formula

$$
E_{i j}=\sum_{n \in \mathbb{Z}^{*}}: \psi_{-n}^{+, i} \psi_{n}^{-, j}:-\sum_{r \in 1 / 2+\mathbb{Z}}: \gamma_{-r}^{+, i} \gamma_{r}^{-, j}: .
$$

For $j \in \mathbb{N}$, we define the $d \times d$ matrices $X^{-j}$ by

$$
X^{-j}:=\left(\begin{array}{cccc}
\gamma_{-\frac{1}{2}}^{-, d} & \gamma_{-\frac{1}{2}}^{-, d-1} & \ldots & \gamma_{-\frac{1}{2}}^{-, 1} \\
\vdots & \vdots & \ldots & \vdots \\
\gamma_{-j+\frac{1}{2}}^{-, d} & \gamma_{-j+\frac{1}{2}}^{-, d-1} & \cdots & \gamma_{-j+\frac{1}{2}}^{-, 1} \\
\psi_{-j}^{-, d} & \psi_{-j}^{-, j-1} & \ldots & \psi_{-j}^{-, 1} \\
\vdots & \vdots & \ldots & \vdots \\
\psi_{-j}^{-, d} & \psi_{-j}^{-, d-1} & \ldots & \psi_{-j}^{-, 1}
\end{array}\right) .
$$

Note that $X^{-j}=X^{-d}$ for all $j \geq d$. The matrices $X^{j}$, for $j \in \mathbb{N}$, are defined similarly. Namely, $X^{j}$ is obtained from $X^{-j}$ by replacing $\psi_{i}^{-, k}$ by $\psi_{i}^{+, d-k+1}$ and $\gamma_{r}^{-, k}$ by $\gamma_{r}^{+, d-k+1}$. For $0 \leq r \leq d$ and $i \in \mathbb{Z}^{*}$, we let $X_{r}^{i}$ denote the first $r \times r$ minor of the matrix $X^{i}$. (We use the definition given in [5] for the determinant of a matrix with Grassmannian entries.)

Given a generalized partition $\lambda=\left(\lambda_{1}, \lambda_{2}, \cdots, \lambda_{d}\right)$ of length $d$, we have the shifted Frobenius notations $\Lambda\left(\lambda^{+}\right)=\left(\xi_{\frac{1}{2}}^{+}, \xi_{1 \frac{1}{2}}^{+}, \cdots, \xi_{r-\frac{1}{2}}^{+} \mid \xi_{1}^{+}, \xi_{2}^{+}, \cdots, \xi_{r}^{+}\right)$and $\Lambda\left(\lambda^{-*}\right)=$ $\left(\xi_{\frac{1}{2}}^{-}, \xi_{1 \frac{1}{2}}^{-}, \cdots, \xi_{s-\frac{1}{2}}^{-} \mid \xi_{1}^{-}, \xi_{2}^{-}, \cdots, \xi_{s}^{-}\right)$for the partitions $\lambda^{+}$and $\lambda^{-*}$, respectively (see Section 2.1). We let $\Lambda^{\widehat{\mathcal{A}}}(\lambda)$ be an element of the dual space $\left(\widehat{\mathcal{A}}_{0}\right)^{*}$ of $\widehat{\mathcal{A}}_{0}$ defined by

$$
\Lambda^{\widehat{\mathcal{A}}}(\lambda):=\sum_{j \leq r, j \in \frac{1}{2} \mathbb{N}} \xi_{j}^{+} \omega_{j}-\sum_{j \leq s, j \in \frac{1}{2} \mathbb{N}} \xi_{j}^{-} \omega_{j}+d \Lambda_{0} .
$$

We have the following result, which is analogous to the $\left(\widehat{\mathrm{gl}}_{\infty \mid \infty}, g l_{d}\right)$-duality of [7].

Theorem 5.2. The Lie superalgebra $\widehat{\mathcal{A}}$ and $g l_{d}$ form a dual pair on $\mathfrak{F}_{0}^{d}$ in the sense of Howe. In particular, we have the following (multiplicity-free) decomposition of $\mathfrak{F}_{0}^{d}$ with respect to their joint action

$$
\mathfrak{F}_{0}^{d} \cong \sum_{\lambda} L\left(\widehat{\mathcal{A}}, \Lambda^{\widehat{\mathcal{A}}}(\lambda)\right) \otimes V_{d}^{\lambda},
$$

where $\lambda$ runs over all generalized partitions of length $d$. Furthermore, the following vector

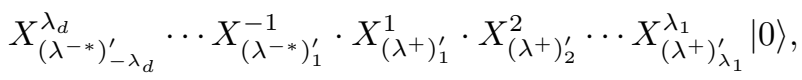

is a joint highest weight vector of the $\lambda$-component.

5.3. Free field realizations of $\widehat{\complement}$ and $(\widehat{\mathcal{C}}, S p(2 d))$-duality. Let us first recall some facts about the complex symplectic group $S p(2 d)$ (see, e.g., 2, 10, 11]), which we take to be the subgroup of $G L(2 d)$ preserving the non-degenerate skew-symmetric bilinear form on $\mathbb{C}^{2 d}$ given by

$$
J_{2 d}=\left(\begin{array}{cc}
0 & I_{d} \\
-I_{d} & 0
\end{array}\right)
$$

where $I_{d}$ is the $d \times d$ identity matrix. More explicitly, $S p(2 d)$ consists of those $A \in$ $G L(2 d)$ such that $A^{t} J_{2 d} A=J_{2 d}$, where $A^{t}$ is the transpose of the matrix $A$. The 
Lie algebra $\mathfrak{s p}(2 d)$ of $S p(2 d)$ consists of those $X \in g l(2 d)$ with $X^{t} J_{2 d}+J_{2 d} X=0$. Denote by $e_{i j}$ the $2 d \times 2 d$ matrix units. Then $\mathfrak{h}:=\sum_{1 \leq i \leq d} \mathbb{C}\left(e_{i i}-e_{d+i, d+i}\right)$ is a Cartan subalgebra, while $\mathfrak{b}:=\sum_{i \leq j \leq d} \mathbb{C}\left(e_{i j}-e_{j+d, i+d}\right)+\sum_{i \leq j} \mathbb{C}\left(e_{i, j+d}+e_{j+d, i}\right)$ is the standard Borel subalgebra containing $\mathfrak{h}$. Let $h_{i}=e_{i i}-e_{d+i, d+i}$.

We write an element $\lambda \in \mathfrak{h}^{*}$ as $\lambda=\left(\lambda_{1}, \lambda_{2}, \cdots, \lambda_{d}\right)$ where $\lambda_{i}=\lambda\left(h_{i}\right)$ for $i=1,2, \cdots, d$. Let $V_{\mathfrak{s p}(2 d)}^{\lambda}$ denote the irreducible $\mathfrak{s p}(2 d)$-module with highest weight $\lambda \in \mathfrak{h}^{*}$ defined with respect to the standard Borel subalgebra. Then $V_{\mathfrak{s p}(2 d)}^{\lambda}$ is finite-dimensional if and only if $\lambda_{1} \geq \lambda_{2} \geq \cdots \geq \lambda_{d}$ and $\lambda_{i} \in \mathbb{Z}_{+}$for $i=1, \cdots, d$. Furthermore, every such representation lifts to a unique irreducible representation of $S p(2 d)$, which is denoted by $V_{S p(2 d)}^{\lambda}$ and so we obtain an obvious parametrisation of $S p(2 d)$-highest weights in terms of Young diagrams $\lambda$ with $l(\lambda) \leq d$.

Introduce the following operators on the Fock space $\mathfrak{F}_{0}^{d}$ :

$$
\begin{aligned}
& E_{i j}^{s p+}=\sum_{n \in \mathbb{N}}: \psi_{-n}^{+, i} \psi_{n}^{+, j}:-\sum_{n \in-\mathbb{N}}: \psi_{-n}^{+, i} \psi_{n}^{+, j}:+\sum_{r \in 1 / 2+\mathbb{Z}}: \gamma_{-r}^{+, i} \gamma_{r}^{+, j}:, \\
& E_{i j}^{s p-}=\sum_{n \in \mathbb{N}}: \psi_{-n}^{-, i} \psi_{n}^{-, j}:-\sum_{n \in-\mathbb{N}}: \psi_{-n}^{-, i} \psi_{n}^{-, j}:-\sum_{r \in 1 / 2+\mathbb{Z}}: \gamma_{-r}^{-, i} \gamma_{r}^{-, j}:,
\end{aligned}
$$

where $1 \leq i, j \leq d$. It is clear that (5.5) and (5.6) together with (5.4) span the Lie algebra $\mathfrak{s p}(2 d)$. Its action on $\mathfrak{F}_{0}^{d}$ can be lifted to an action of Lie group $S p(2 d)$. Moreover, $\mathfrak{F}_{0}^{d}$ is a direct sum of finite dimensional irreducible $S p(2 d)$-modules.

On the other hand, $S p(2 d)$ acts on $\mathscr{A}_{0}^{d}$ by conjugation. It is not hard to see that the $S p(2 d)$-invariants of the associative algebra $\mathscr{A}_{0}^{d}$ is generated by the following combinations of elements of (5.2) and (5.3):

$$
\begin{array}{rlr}
(5.7) & C & \tilde{e}_{r, s}=e_{r, s}-e_{-s,-r} \\
(5.8) & \tilde{e}_{i, j}=e_{i, j}-e_{-j,-i}, \quad i j>0 ; & \tilde{e}_{i, j}=e_{i, j}+e_{-j,-i}, \quad i j<0 \\
(5.9) & \tilde{e}_{i, r}=\tilde{e}_{-r,-i}=e_{i, r}+e_{-r,-i}, i>0 ; & \tilde{e}_{i, r}=-\tilde{e}_{-r,-i}=e_{i, r}-e_{-r,-i}, i<0
\end{array}
$$

where $i, j \in \mathbb{Z}^{*}$ and $r, s \in \frac{1}{2}+\mathbb{Z}$. Note that (5.7), (5.8) and (5.9) span the Lie superalgebra $\widehat{\mathrm{C}}^{f}$ and hence $S p(2 d)$ commutes with $\widehat{\mathrm{C}}$.

For any given partition $\lambda=\left(\lambda_{1}, \lambda_{2}, \cdots, \lambda_{d}\right)$ of length $d$, we have the shifted Frobenius notation $\Lambda(\lambda)=\left(\xi_{\frac{1}{2}}, \xi_{\frac{3}{2}}, \cdots, \xi_{r-\frac{1}{2}} \mid \xi_{1}, \xi_{2}, \cdots, \xi_{r}\right)$ (see Section 2.1). Let $\Lambda^{\widehat{\mathrm{e}}}(\lambda) \in \widehat{\mathcal{C}}_{0}^{*}$ be defined by

$$
\Lambda^{\widehat{\mathrm{e}}}(\lambda):=\sum_{j \leq r, j \in \frac{1}{2} \mathbb{N}} \xi_{j} \omega_{j}+d \Lambda_{0} .
$$

Let $\lambda$ be a partition of length $d$. Then the vector

$$
X_{\lambda_{1}^{\prime}}^{1} \cdot X_{\lambda_{2}^{\prime}}^{2} \cdots X_{\lambda_{\lambda_{1}}^{\prime}}^{\lambda_{1}}|0\rangle
$$

is a highest weight vector of the Lie superalgebra $\widehat{\mathcal{C}}$ with highest weight $\Lambda^{\widehat{e}}(\lambda)$ and it is also a highest weight vector of $S p(2 d)$. By using similar arguments as in 7 (see also [24, 3]), we have the following theorem.

Theorem 5.3. The Lie superalgebra $\widehat{\mathcal{C}}$ and $S p(2 d)$ form a dual pair on $\mathfrak{F}_{0}^{d}$ in the sense of Howe, namely, there exists the following (multiplicity-free) decomposition of $\mathfrak{F}_{0}^{d}$ with respect to their joint action

$$
\mathfrak{F}_{0}^{d} \cong \sum_{\lambda} L\left(\widehat{\mathcal{C}}, \Lambda^{\widehat{\mathrm{e}}}(\lambda)\right) \otimes V_{S p(2 d)}^{\lambda},
$$


where the summation is over all partitions of length $d$. Futhermore, the vector

$$
X_{\lambda_{1}^{\prime}}^{1} \cdot X_{\lambda_{2}^{\prime}}^{2} \cdots X_{\lambda_{\lambda_{1}}^{\prime}}^{\lambda_{1}}|0\rangle
$$

is a $\widehat{\mathrm{C}} \times S p(2 d)$ highest weight vector of the $\lambda$-component.

5.4. Fock space realizations of $\widehat{\mathcal{D}}$. In this subsection we will construct two types of free field realizations of $\widehat{\mathcal{D}}$ which are respectively associated with the $(\widehat{\mathcal{D}}, O(2 d))$ and $(\widehat{\mathcal{D}}, O(2 d+1))$-dualities.

5.4.1. Basic facts on representations of the complex orthogonal group. We start by recalling some facts about finite dimensional representations of the complex orthogonal group $O(k)$ (see, e.g., 2, 10, 11). We first consider the case when $k=2 d$ is even. Let $\left\{e^{1}, e^{2}, \cdots, e^{2 d}\right\}$ be the standard basis for $\mathbb{C}^{2 d}$. We consider the complex orthogonal group $O(2 d)$ defined with respect to the non-degenerate symmetric bilinear form on $\mathbb{C}^{2 d}$ given by

$$
K_{2 d}=\left(\begin{array}{cc}
0 & I_{d} \\
I_{d} & 0
\end{array}\right)
$$

Denote by $\mathfrak{s o}(2 d)$ the Lie algebra of $O(2 d)$. Let $h_{i}:=e_{i i}-e_{d+i, d+i}, E_{i j}^{s o+}:=$ $e_{i, j+d}-e_{j, i+d}$ and $E_{i j}^{s o-}:=e_{i+d, j}-e_{j+d, i}$ for $1 \leq i, j \leq d$. Then $\mathfrak{h}:=\sum_{1 \leq i<d} \mathbb{C} h_{i}$ is a Cartan subalgebra, while $\mathfrak{b}:=\sum_{1 \leq i \leq j \leq d} \mathbb{C}\left(e_{i, j}-e_{j+d, i+d}\right)+\sum_{1 \leq i, j \leq d} \mathbb{C} E_{i j}^{s o+}$ is the standard Borel subalgebra containing $\mathfrak{h}$.

Write an element $\lambda \in \mathfrak{h}^{*}$ as $\lambda=\left(\lambda_{1}, \lambda_{2}, \cdots, \lambda_{d}\right)$, where $\lambda_{i}=\lambda\left(h_{i}\right)$, for $i=$ $1,2, \cdots, d$. Let $V_{\mathfrak{s o}(2 d)}^{\lambda}$ denote the irreducible highest weight $\mathfrak{s o}(2 d)$-module with highest weight $\lambda \in \mathfrak{h}^{*}$ defined with respect to the standard Borel subalgebra. Then $V_{\mathfrak{s o}(2 d)}^{\lambda}$ is finite dimensional if and only if $\lambda_{1} \geq \lambda_{2} \geq \cdots \geq\left|\lambda_{d}\right|$ with either $\lambda_{i} \in \mathbb{Z}$ or else $\lambda_{i} \in \frac{1}{2}+\mathbb{Z}$ for all $i=1, \cdots, d$. Furthermore, the $\mathfrak{s o}(2 d)$-module $V_{\mathfrak{s o}(2 d)}^{\lambda}$ lifts to an $S O(2 d)$-module if and only if $\lambda_{1} \geq \lambda_{2} \geq \cdots \geq\left|\lambda_{d}\right|$ with $\lambda_{i} \in \mathbb{Z}$ for all $i=1, \cdots, d$.

When a finite-dimensional irreducible $O(2 d)$-module $V$ is regarded as an $\mathfrak{s o}(2 d)$ module, either

(i) $V$ is a direct sum of two irreducible $\mathfrak{s o}(2 d)$-modules with integral highest weights $\left(\lambda_{1}, \lambda_{2}, \cdots, \lambda_{d}\right)$ and $\left(\lambda_{1}, \lambda_{2}, \cdots, \lambda_{k-1},-\lambda_{d}\right)$ respectively, where $\lambda_{k}>0$; or

(ii) $V$ is an irreducible $\mathfrak{s o}(2 d)$-module with integral highest weight of the form $\left(\lambda_{1}, \lambda_{2}, \cdots, \lambda_{d-1}, 0\right)$.

In case (i), we denote $V$ by $W_{O(2 d)}^{\widetilde{\lambda}}$, where $\widetilde{\lambda}=\left(\lambda_{1}, \lambda_{2}, \cdots, \lambda_{d-1}, \lambda_{d}>0\right)$. In case (ii), there are two possible choices of $V$, which we denote by $W_{O(2 d)}^{\widetilde{\lambda}}$ and $W_{O(2 d)}^{\tilde{\lambda}} \otimes$ det, respectively. Recalling that $O(2 d)$ is a semidirect product of $S O(2 d)$ and $\mathbb{Z}_{2}$. Thus the $O(2 d)$-modules $W_{O(2 d)}^{\tilde{\lambda}}$ and $W_{O(2 d)}^{\tilde{\lambda}} \otimes$ det restrict to isomorphic $S O(2 d)$ modules. However, as $O(2 d)$-modules they differ by the determinant representation so that we may distinguish these two modules as follows: consider the element $\tau \in O(2 d)-S O(2 d)$ that switches the basis vector $e^{d}$ with $e^{2 d}$ and leaves all other basis vectors of $\mathbb{C}^{2 d}$ invariant. We declare $W_{O(2 d)}^{\tilde{\lambda}}$ to be the $O(2 d)$-module, where $\tau$ acts on any $S O(d)$-highest weight vector trivially. Note that $\tau$ acts on an $S O(2 d)$ highest weight vector in the $O(2 d)$-module $W_{O(2 d)}^{\tilde{\lambda}} \otimes \operatorname{det}$ by -1 . 
We may associate Young diagrams $\lambda$ of length $2 d$ to these integral highest weights of $O(2 d)$ as follows (cf. [14]). For $\lambda_{1} \geq \lambda_{2} \geq \cdots \geq \lambda_{d}>0$ with $\lambda_{i} \in$ $\mathbb{Z}_{+}$for all $i$, we have an obvious Young diagram of length $2 d$ by putting $\lambda:=$ $\left(\lambda_{1}, \lambda_{2}, \cdots, \lambda_{d}, 0, \cdots, 0\right)$. When $\lambda_{d}=0$, we associate to the highest weight of $W_{O(2 d)}^{\tilde{\lambda}}$ the usual Young diagram of length $2 d$ by putting

$$
\lambda:=\left(\lambda_{1}, \lambda_{2}, \cdots, \lambda_{d}, 0, \cdots, 0\right) .
$$

We put $V_{O(2 d)}^{\lambda}:=W_{O(2 d)}^{\tilde{\lambda}}$. To the highest weight of $W_{O(2 d)}^{\tilde{\lambda}} \otimes$ det we associate the Young diagram $\bar{\lambda}$ obtained from $\lambda$ by replacing its first column by a column of length $2 d-\lambda_{1}^{\prime}$. Let $V_{O(2 d)}^{\bar{\lambda}}:=W_{O(2 d)}^{\widetilde{\lambda}} \otimes$ det.

Hereafter, we shall adopt the following convention. Given any partition $\lambda$ of length $2 d$ satisfying the condition $\lambda_{1}^{\prime}+\lambda_{2}^{\prime} \leq 2 d$, we denote by $\bar{\lambda}$ the partition obtained from $\lambda$ by replacing its first column by a column of length $2 d-\lambda_{1}^{\prime}$. There is a one-to-one correspondence between the finite dimensional irreducible $O(2 d)$ representations and the partitions $\lambda$ of length $2 d$ satisfying the condition $\lambda_{1}^{\prime}+\lambda_{2}^{\prime} \leq$ $2 d$.

Next consider the case when $k=2 d+1$ is odd, where the orthogonal group $O(2 d+1)$ is defined with respect to the following non-degenerate symmetric bilinear form on $\mathbb{C}^{2 d+1}$

$$
K_{2 d+1}=\left(\begin{array}{ccc}
0 & 0 & I_{d} \\
0 & 1 & 0 \\
I_{d} & 0 & 0
\end{array}\right) .
$$

Denote by $\mathfrak{s o}(2 d+1)$ the Lie algebra of $O(2 d+1)$. Let $e_{i j}$ be the $(2 d+1) \times(2 d+1)$ matrix units. Set $h_{i}:=e_{i i}-e_{d+1+i, d+1+i}, E_{i}^{s o+}:=e_{i, d+1}-e_{d+1, d+1+i}, E_{j}^{s o-}:=$ $e_{d+1, j}-e_{j+d+1, d+1}, E_{i j}^{s o+}:=e_{i, j+d+1}-e_{j, i+d+1}$ and $E_{i j}^{s o-}:=e_{i+d+1, j}-e_{j+d+1, i}$ for $1 \leq i, j \leq d$. Then $\mathfrak{h}:=\sum_{1 \leq i \leq d} \mathbb{C} h_{i}$ is a Cartan subalgebra, while $\mathfrak{b}:=$ $\sum_{1 \leq i \leq j \leq d} \mathbb{C}\left(e_{i, j}-e_{j+d+1, i+d+1}\right)+\sum_{1 \leq i \leq d} \mathbb{C} E_{i}^{s o+}+\sum_{1 \leq i, j \leq d} \mathbb{C} E_{i j}^{s o+}$ is the standard Borel subalgebra containing $\mathfrak{h}$.

Write an element $\lambda \in \mathfrak{h}^{*}$ as $\lambda=\left(\lambda_{1}, \lambda_{2}, \cdots, \lambda_{d}\right)$, where $\lambda_{i}=\lambda\left(h_{i}\right)$, for $i=$ $1,2, \cdots, d$. Let $V_{\mathfrak{s o}(2 d+1)}^{\lambda}$ denote the irreducible highest weight $\mathfrak{s o}(2 d+1)$-module with highest weight $\lambda \in \mathfrak{h}^{*}$ defined with respect to the standard Borel subalgebra. Then $V_{\mathfrak{s o}(2 d+1)}^{\lambda}$ is finite dimensional if and only if $\lambda_{1} \geq \lambda_{2} \geq \cdots \geq \lambda_{d}$ with either $\lambda_{i} \in \mathbb{Z}_{+}$or $\lambda_{i} \in \frac{1}{2}+\mathbb{Z}_{+}$for all $i=1, \cdots, d$. Furthermore, $V_{\mathfrak{s o}(2 d+1)}^{\lambda}$ lifts to a representation of $S O(2 d+1)$ if and only if $\lambda_{i} \in \mathbb{Z}_{+}$.

Recall that $O(2 d+1)$ is a direct product of $S O(2 d+1)$ and $\mathbb{Z}_{2}$. Thus every finite-dimensional irreducible representation of $O(2 d+1)$ restricts to an irreducible representation of $S O(2 d+1)$. Conversely, an irreducible $S O(2 d+1)$-module gives rise to two non-isomorphic $O(2 d+1)$-modules that differ from each other by the determinant representation det. We let $W_{O(2 d+1)}^{\widetilde{\lambda}}$ stand for the irreducible $O(2 d+1)$ module corresponding to $\tilde{\lambda}=\left(\lambda_{1} \geq \lambda_{2} \geq \cdots \geq \lambda_{k} \geq 0\right)$ on which the element $-I_{2 d+1} \in O(2 d+1)$ acts trivially, where $I_{2 d+1}$ is the identity $(2 d+1) \times(2 d+1)$ matrix. Then $\left\{W_{O(2 d+1)}^{\tilde{\lambda}}, W_{O(2 d+1)}^{\tilde{\lambda}} \otimes \operatorname{det}\right\}$ with $\widetilde{\lambda}$ ranging over all partitions with length $d$ as above is a complete set of finite-dimensional non-isomorphic irreducible $O(2 d+1)$-modules.

Similarly as before we may associate Young diagrams of length $2 d+1$ to these $O(2 d+1)$-highest weights. For the highest weight $\tilde{\lambda}=\left(\lambda_{1} \geq \lambda_{2} \cdots \geq \lambda_{d} \geq 0\right)$ 
of $W_{O(2 d+1)}^{\widetilde{\lambda}}$, we have an obvious Young diagram $\lambda=\left(\lambda_{1}, \lambda_{2}, \cdots, \lambda_{d}, 0, \cdots, 0\right)$ of length $2 d+1$. Let $V_{O(2 d+1)}^{\lambda}:=W_{O(2 d+1)}^{\tilde{\lambda}}$. To the highest weight of $W_{O(2 d+1)}^{\tilde{\lambda}} \otimes \operatorname{det}$ we associate the Young diagram $\bar{\lambda}$ obtained from $\lambda$ by replacing its first column by a column of length $2 d+1-\lambda_{1}^{\prime}$. (Hereafter, $\bar{\lambda}$ will always denote a partition obtained in this way from $\lambda$.) In this case, we let $V_{O(2 d+1)}^{\bar{\lambda}}:=W_{O(2 d+1)}^{\widetilde{\lambda}} \otimes$ det. Now there is a oneto-one correspondence between the finite dimensional irreducible representations of $O(2 d+1)$ and the partitions $\mu$ of length $2 d+1$ satisfying $\mu_{1}^{\prime}+\mu_{2}^{\prime} \leq 2 d+1$.

5.4.2. Free field realizations of $\widehat{\mathcal{D}}$ and $(\widehat{\mathcal{D}}, O(2 d))$-duality. Let us consider the realization of $\widehat{\mathcal{D}}$ on the Fock space $\mathfrak{F}_{0}^{d}$ related to the $(\widehat{\mathcal{D}}, O(2 d))$-duality. Introduce the following operators on $\mathfrak{F}_{0}^{d}(1 \leq i, j \leq d)$ :

$$
\begin{aligned}
& E_{i j}^{s o+}=\sum_{n \in \mathbb{Z}^{*}}: \psi_{-n}^{+, i} \psi_{n}^{+, j}:+\sum_{r \in 1 / 2+\mathbb{Z}_{+}}: \gamma_{-r}^{+, i} \gamma_{r}^{+, j}:-\sum_{r \in-1 / 2-\mathbb{Z}_{+}}: \gamma_{-r}^{+, i} \gamma_{r}^{+, j}:, \\
& E_{i j}^{s o-}=\sum_{n \in \mathbb{Z}^{*}}: \psi_{-n}^{-, i} \psi_{n}^{-, j}:-\sum_{r \in 1 / 2+\mathbb{Z}_{+}}: \gamma_{-r}^{-, i} \gamma_{r}^{-, j}:+\sum_{r \in-1 / 2-\mathbb{Z}_{+}}: \gamma_{-r}^{-, i} \gamma_{r}^{-, j}:,
\end{aligned}
$$

which together with (5.4) span the Lie algebra $\mathfrak{s o}(2 d)$. The action of the Lie algebra $\mathfrak{s o}(2 d)$ on $\mathfrak{F}_{0}^{d}$ can be lifted to an action of the Lie group $S O(2 d)$, which can in turn be extended to an action of $O(2 d)$. Moreover, $\mathfrak{F}_{0}^{d}$ is a direct sum of finite dimensional irreducible modules over $O(2 d)$.

Note that $O(2 d)$ acts on the associative algebra $\mathscr{A}_{0}^{d}$ by conjugation, and the $O(2 d)$-invariants in $\mathscr{A}_{0}^{d}$ are generated by the following combinations of elements of (5.2) and (5.3):

$$
\tilde{e}_{r, s}=e_{r, s}-e_{-s,-r}, r s>0 ; \quad \tilde{e}_{r, s}=e_{r, s}+e_{-s,-r}, r s<0
$$

$$
\tilde{e}_{i, r}=\tilde{e}_{-r,-i}=e_{i, r}+e_{-r,-i}, r>0 ; \quad \tilde{e}_{i, r}=-\tilde{e}_{-r,-i}=e_{i, r}-e_{-r,-i}, r<0,
$$

where $i, j \in \mathbb{Z}^{*}$ and $r, s \in \frac{1}{2}+\mathbb{Z}$. Note that (5.13), (5.14) and (5.15) span the Lie superalgebra $\widehat{\mathcal{D}}^{f}$ in $\mathscr{A}_{0}^{d}$.

For each $j \in\{1,2, \cdots, d\}$, we define the $d \times d$ matrix $\tilde{X}^{j}$ as follows:

$$
\tilde{X}^{j}:=\left(\begin{array}{ccccc}
\gamma_{-\frac{1}{2}}^{+, 1} & \gamma_{-\frac{1}{2}}^{+, 2} & \cdots & \gamma_{-\frac{1}{2}}^{+, d-1} & \gamma_{-\frac{1}{2}}^{-, d} \\
\gamma_{-\frac{3}{2}}^{+, 1} & \gamma_{-\frac{3}{2}}^{+, 2} & \cdots & \gamma_{-\frac{3}{2}}^{+, d} & \gamma_{-\frac{3}{2}}^{-, d} \\
\vdots & \vdots & \cdots & \vdots & \vdots \\
\gamma_{-\frac{2 j-1}{+, 1}}^{+,} & \gamma_{-\frac{2 j-1}{+, 2}}^{+, 2} & \cdots & \gamma_{-\frac{2 j-1}{+, d}}^{+, d} & \gamma_{-\frac{2 j-1}{-, d}}^{-, d} \\
\psi_{-j}^{+, 1} & \psi_{-j}^{+, 2} & \cdots & \psi_{-j}^{+, d^{2}-1} & \psi_{-j}^{-, d} \\
\vdots & \vdots & \cdots & \vdots & \vdots \\
\psi_{-j}^{+, 1} & \psi_{-j}^{+, 2} & \cdots & \psi_{-j}^{+, d-1} & \psi_{-j}^{-, d}
\end{array}\right) .
$$

For any integer $j \geq d, \widetilde{X}^{j}:=\widetilde{X}^{d}$. Note that the matrix $\widetilde{X}^{j}$ is obtained from $X^{j}$ by replacing its last column by $\left(\gamma_{-\frac{1}{2}}^{-, d}, \gamma_{-\frac{3}{2}}^{-, d}, \cdots, \gamma_{-\frac{2 j-1}{2}}^{-, d} \psi_{-j}^{-, d} \cdots \psi_{-j}^{-, d}\right)$. For $0 \leq r \leq d$ and $i>0$, we let $\widetilde{X}_{r}^{i}$ denote the first $r \times r$ minor of the matrix $\widetilde{X}^{i}$. 
We define the $2 d \times 2 d$ matrix $\Gamma$ as follows:

$$
\Gamma:=\left(\begin{array}{cccccccc}
\gamma_{-1}^{+, 1} & \gamma_{-1}^{+, 2} & \cdots & \gamma_{-\frac{1}{2}}^{+, d} & \gamma_{-\frac{1}{2}}^{-, d} & \gamma_{-\frac{1}{2}}^{-, d-1} & \cdots & \gamma_{-\frac{1}{2}}^{-, 1} \\
\psi_{-1}^{+, 1} & \psi_{-1}^{+, 2} & \cdots & \psi_{-1}^{+, d} & \psi_{-1}^{-, d} & \psi_{-1}^{-, d-1} & \cdots & \psi_{-1}^{-, 1} \\
\vdots & \vdots & \cdots & \vdots & \vdots & \vdots & \cdots & \vdots \\
\psi_{-1}^{+, 1} & \psi_{-1}^{+, 2} & \cdots & \psi_{-1}^{+, d} & \psi_{-1}^{-, d} & \psi_{-1}^{-, d-1} & \cdots & \psi_{-1}^{-, 1}
\end{array}\right)
$$

For $r>d$, we let $\Gamma_{r}$ denote the first $r \times r$ minor of the matrix $\Gamma$.

Let $\Lambda(\lambda)=\left(\xi_{\frac{1}{2}}, \xi_{1 \frac{1}{2}}, \cdots, \xi_{r-\frac{1}{2}} \mid \xi_{1}, \xi_{2}, \cdots, \xi_{r}\right)$ be the shifted Frobenius notation (see Section 2.1) for the partition $\lambda=\left(\lambda_{1}, \lambda_{2}, \cdots, \lambda_{2 d}\right)$ of length $2 d$ with $\lambda_{1}^{\prime}+\lambda_{2}^{\prime} \leq$ $2 d$. Let $\Lambda^{\widehat{\mathcal{D}}}(\lambda) \in \widehat{\mathcal{D}}_{0}^{*}$ be defined by

$$
\Lambda^{\widehat{\mathcal{D}}}(\lambda):=\sum_{j \leq r, j \in \frac{1}{2} \mathbb{N}} \xi_{j} \omega_{j}+d \Lambda_{0}
$$

By using similar arguments as in 7] (see also [24, 3]) and the explicit construction of the joint highest weight vectors of $\widehat{\mathcal{D}}$ and $S O(2 d)$, we have the following theorem.

Theorem 5.4. The Lie superalgebra $\widehat{\mathcal{D}}$ and $O(2 d)$ form a dual pair on $\mathfrak{F}_{0}^{d}$ in the sense of Howe, namely, we have the following multiplicity-free decomposition of $\mathfrak{F}_{0}^{d}$ with respect to their joint action

$$
\mathfrak{F}^{d} \cong \sum_{\lambda} L\left(\widehat{\mathcal{D}}, \Lambda^{\widehat{\mathcal{D}}}(\lambda)\right) \otimes V_{O(2 d)}^{\lambda},
$$

where the summation is over all partitions of length $2 d$ with $\lambda_{1}^{\prime}+\lambda_{2}^{\prime} \leq 2 d$. Furthermore, the following vectors are $\widehat{\mathcal{D}} \times \mathfrak{s o}(2 d)$ highest weight vectors:

(i) $X_{\lambda_{1}^{\prime}}^{1} X_{\lambda_{2}^{\prime}}^{2} \cdots X_{\lambda_{\lambda_{1}}^{\prime}}^{\lambda_{1}}|0\rangle$ when $\lambda_{1}^{\prime}<d$;

(ii) $X_{\lambda_{1}^{\prime}}^{1} X_{\lambda_{2}^{\prime}}^{2} \cdots X_{\lambda_{\lambda_{1}}^{\prime}}^{\lambda_{1}}|0\rangle$ and $\widetilde{X}_{\lambda_{1}^{\prime}}^{1} \widetilde{X}_{\lambda_{2}^{\prime}}^{2} \cdots \widetilde{X}_{\lambda_{\lambda_{1}}^{\prime}}^{\lambda_{1}}|0\rangle$ when $\lambda_{1}^{\prime}=d$;

(iii) $\Gamma_{\lambda_{1}^{\prime}} X_{\lambda_{2}^{\prime}}^{2} \cdots X_{\lambda_{\lambda_{1}}^{\prime}}^{\lambda_{1}}|0\rangle$ when $\lambda_{1}^{\prime}>d$.

5.4.3. Free field realizations of $\widehat{\mathcal{D}}$ and $(\widehat{\mathcal{D}}, O(2 d+1))$-duality. Now we turn to the free field realization of $\widehat{\mathcal{D}}$ associated with the $(\widehat{\mathcal{D}}, O(2 d+1))$-duality. Introduce a free fermionic field $\phi(z):=\sum_{n \in \mathbb{Z}^{*}} \phi_{n} z^{-n-1}$ and a free bosonic field $\chi(z):=$ $\sum_{r \in \frac{1}{2}+\mathbb{Z}} \chi_{r} z^{-r-1 / 2}$ with the non-trivial anti-commutation relations $\left[\phi_{m}, \phi_{n}\right]=$ $\delta_{i j} \delta_{m+n, 0}$ and commutation relations $\left[\chi_{r}, \chi_{s}\right]=\delta_{i j} \delta_{r+s, 0}$ for $r>0$. We shall denote by $\mathscr{A}_{0}^{d+\frac{1}{2}}$ the associative superalgebra generated by the modes of all the quantum fields $\widetilde{\psi}^{ \pm, i}(z), \gamma^{ \pm, i}(z), i=1, \cdots, d, \phi(z)$, and $\chi(z)$. Let $\mathfrak{F}_{0}^{d+\frac{1}{2}}$ denote the Fock space of the quantum fields generated by the vacuum vector $|0\rangle$, where $\psi_{m}^{ \pm, i}|0\rangle=\gamma_{r}^{ \pm, i}|0\rangle=\phi_{m}|0\rangle=\chi_{r}|0\rangle=0$, for $i=1,2, \cdots, d, m>0$ and $r>0$. 
We can realize $\widehat{\mathcal{D}}$ with central charge $d+\frac{1}{2}$ on $\mathfrak{F}_{0}^{d+\frac{1}{2}}$ by the following operators $\left(i, j \in \mathbb{Z}^{*}, r, s \in \frac{1}{2}+\mathbb{Z}\right)$ :

$$
\begin{aligned}
& \tilde{e}_{i j}:=\sum_{p=1}^{d}: \psi_{-i}^{+, p} \psi_{j}^{-, p}:-\sum_{p=1}^{d}: \psi_{j}^{+, p} \psi_{-i}^{-, p}:+: \phi_{-i} \phi_{j}: ; \\
& \tilde{e}_{r s}:=-\tilde{e}_{-s,-r}:=-\sum_{p=1}^{d}: \gamma_{-r}^{+, p} \gamma_{s}^{-, p}:+\sum_{p=1}^{d}: \gamma_{s}^{+, p} \gamma_{-r}^{-, p}:+: \chi_{-r} \chi_{s}:, r s>0 \\
& \tilde{e}_{r s}:=-\sum_{p=1}^{d}: \gamma_{-r}^{+, p} \gamma_{s}^{-, p}:-\sum_{p=1}^{d}: \gamma_{s}^{+, p} \gamma_{-r}^{-, p}:+: \chi_{-r} \chi_{s}:, r<0, s>0 \\
& \tilde{e}_{r s}:=-\sum_{p=1}^{d}: \gamma_{-r}^{+, p} \gamma_{s}^{-, p}:-\sum_{p=1}^{d}: \gamma_{s}^{+, p} \gamma_{-r}^{-, p}:-: \chi_{-r} \chi_{s}:, r>0, s<0 \\
& \tilde{e}_{i s}:=\tilde{e}_{-s,-i}:=\sum_{p=1}^{d}: \psi_{-i}^{+, p} \gamma_{s}^{-, p}:-\sum_{p=1}^{d}: \gamma_{s}^{+, p} \psi_{-i}^{-, p}:+: \phi_{-i} \chi_{s}:, s>0 \\
& \tilde{e}_{i s}:=-\tilde{e}_{-s,-i}:=\sum_{p=1}^{d}: \psi_{-i}^{+, p} \gamma_{s}^{-, p}:+\sum_{p=1}^{d}: \gamma_{s}^{+, p} \psi_{-i}^{-, p}:-: \phi_{-i} \chi_{s}:, s<0 .
\end{aligned}
$$

Introduce the following operators on the Fock space $\mathfrak{F}_{0}^{d+\frac{1}{2}}$ :

$$
\begin{aligned}
& E_{i}^{s o+}=\sum_{n \in \mathbb{Z}^{*}}: \phi_{-n} \psi_{n}^{+, i}:-\sum_{r \in 1 / 2+\mathbb{Z}_{+}}: \chi_{-r} \gamma_{r}^{+, i}:+\sum_{r \in-1 / 2-\mathbb{Z}_{+}}: \chi_{-r} \gamma_{r}^{+, i}:, \\
& E_{j}^{s o-}=\sum_{n \in \mathbb{Z}^{*}}: \phi_{-n} \psi_{n}^{-, j}:+\sum_{r \in 1 / 2+\mathbb{Z}^{*}}: \chi_{-r} \gamma_{r}^{-, j}:
\end{aligned}
$$

where $1 \leq i, j \leq d$. Note that the operators (5.4), (5.11) and (5.12) act naturally on the Fock space $\mathfrak{F}_{0}^{d+\frac{1}{2}}$. It is easy to see that (5.4), (5.11) and (5.12) together with (5.16) and (5.17) span the Lie algebra $\mathfrak{s o}(2 d+1)$. The action of $\mathfrak{s o}(2 d+1)$ on $\mathfrak{F}_{0}^{d+\frac{1}{2}}$ can be lifted to an action of the Lie group $S O(2 d+1)$, which can further be extended to an action of $O(2 d+1)$. Moreover, $\mathfrak{F}_{0}^{d+\frac{1}{2}}$ is a direct sum of finite dimensional irreducible representations of $O(2 d+1)$. Also it is not hard to see that $O(2 d+1)$ acts on the associative algebra $\mathscr{A}^{d+\frac{1}{2}}$ by conjugation and the $O(2 d+1)$-invariants of $\mathscr{A}^{d+\frac{1}{2}}$ are generated by the operators $\tilde{e}_{r s}$ just described.

We define the $(2 d+1) \times(2 d+1)$ matrix $\widetilde{\Gamma}$ as follows:

$$
\widetilde{\Gamma}:=\left(\begin{array}{ccccccccc}
\gamma_{-\frac{1}{2}}^{+, 1} & \gamma_{-\frac{1}{2}}^{+, 2} & \ldots & \gamma_{-\frac{1}{2}}^{+, d} & \chi_{-\frac{1}{2}} & \gamma_{-\frac{1}{2}}^{-, d} & \gamma_{-\frac{1}{2}}^{-, d-1} & \ldots & \gamma_{-\frac{1}{2}}^{-, 1} \\
\psi_{-1}^{+, 1} & \psi_{-1}^{+, 2} & \ldots & \psi_{-1}^{+, d} & \phi_{-1} & \psi_{-1}^{-, d} & \psi_{-1}^{-, d-1} & \ldots & \psi_{-1}^{-, 1} \\
\vdots & \vdots & \ldots & \vdots & \vdots & \vdots & \vdots & \ldots & \vdots \\
\psi_{-1}^{+, 1} & \psi_{-1}^{+, 2} & \cdots & \psi_{-1}^{+, d} & \phi_{-1} & \psi_{-1}^{-, d} & \psi_{-1}^{-, d-1} & \ldots & \psi_{-1}^{-, 1}
\end{array}\right) .
$$

For $r \in \mathbb{Z}_{+}$, we let $\widetilde{\Gamma}_{r}$ denote the first $r \times r$ minor of the matrix $\widetilde{\Gamma}$.

Using the shifted Frobenius notations $\Lambda(\lambda)=\left(\xi_{\frac{1}{2}}, \xi_{1 \frac{1}{2}}, \cdots, \xi_{r-\frac{1}{2}} \mid \xi_{1}, \xi_{2}, \cdots, \xi_{r}\right)$ for the partition $\lambda=\left(\lambda_{1}, \lambda_{2}, \cdots, \lambda_{2 d+1}\right)$ of length $2 d+1$ with $\lambda_{1}^{\prime}+\lambda_{2}^{\prime} \leq 2 d+1$, we 
define $\Lambda^{\widehat{\mathcal{D}}}(\lambda) \in \widehat{\mathcal{D}}_{0}^{*}$ by

$$
\Lambda^{\widehat{\mathcal{D}}}(\lambda):=\sum_{j \leq r, j \in \frac{1}{2} \mathbb{N}} \xi_{j} \omega_{j}+\frac{2 d+1}{2} \Lambda_{0} .
$$

We have the following theorem.

Theorem 5.5. The Lie superalgebra $\widehat{\mathcal{D}}$ and $O(2 d+1)$ form a dual pair on $\mathfrak{F}_{0}^{d+\frac{1}{2}}$ in the sense of Howe. In particular, we have the following (multiplicity-free) decomposition of $\mathfrak{F}_{0}^{d+\frac{1}{2}}$ with respect to their joint action

$$
\mathfrak{F}_{0}^{d+\frac{1}{2}} \cong \sum_{\lambda} L\left(\widehat{\mathcal{D}}, \Lambda^{\widehat{\mathcal{D}}}(\lambda)\right) \otimes V_{O(2 d+1)}^{\lambda}
$$

where the summation is over all partitions of length $2 d+1$ with $\lambda_{1}^{\prime}+\lambda_{2}^{\prime} \leq 2 d+1$. Furthermore, a joint highest weight vector of the $\lambda$-component with respect to $\widehat{\mathcal{D}} \times$ $S O(2 d+1)$ is given by

$$
\Gamma_{\lambda_{1}^{\prime}} \cdot X_{\lambda_{2}^{\prime}}^{2} \cdots X_{\lambda_{\lambda_{1}}^{\prime}}^{\lambda_{1}}|0\rangle
$$

5.5. Unitarizable modules over $\widehat{\mathrm{gl}}_{\infty \mid \infty}, \widehat{\mathcal{A}}, \widehat{\mathrm{C}}$ and $\widehat{\mathcal{D}}$. Recall that the associative superalgebra $\mathscr{A}^{d}$ generated by the operators $\psi_{m}^{ \pm, k}$ and $\gamma_{-r}^{ \pm, k}$ admits a $*$-structure $\omega: \mathscr{A}^{d} \rightarrow \mathscr{A}^{d}$ defined by

$$
\begin{gathered}
\omega\left(\psi_{m}^{+, k}\right)=\psi_{-m}^{-, k}, \quad \omega\left(\psi_{m}^{-, k}\right)=\psi_{-m}^{+, k}, \\
\omega\left(\gamma_{r}^{+, k}\right)=\left\{\begin{array}{ll}
\gamma_{-r}^{-, k}, & \text { if } r>0, \\
-\gamma_{-r}^{-, k}, & \text { if } r<0 .
\end{array}, \omega\left(\gamma_{r}^{-, k}\right)= \begin{cases}-\gamma_{-r}^{+, k}, & \text { if } r>0, \\
\gamma_{-r}^{+, k}, & \text { if } r<0,\end{cases} \right.
\end{gathered}
$$

for all $m \in \mathbb{Z}, r \in \frac{1}{2}+\mathbb{Z}, k=1,2, \cdots, d$. It can be easily shown that $\omega$ indeed defines an anti-linear anti-involution on $\mathscr{A}^{d}$. Let $\langle\cdot \mid \cdot\rangle$ be the contravariant Hermitian from on the Fock space $\mathfrak{F}^{d}$ defined with respect to $\omega$.

Lemma 5.1. The Fock space $\mathfrak{F}^{d}$ equipped with the contravariant Hermitian from $\langle\cdot \mid \cdot\rangle$ is a unitarizable $\mathscr{A}^{d}$-module.

Proof. In the "particle number basis" for the Fock space, the lemma can be confirmed by a straightforward calculation, details of which are omitted here. However, see Remark 5.1 below.

Remark 5.1. If we remove all the minus signs from (5.19), we still obtain a *structure for $\mathscr{A}^{d}$. In fact, this is the usual conjugation rule for symplectic bosons adopted in the physics literature. However, it is quite well known that the Fock space of symplectic bosons is not unitarizable with respect to the usual conjugation rule.

The anti-linear anti-involution $\omega$ on $\mathscr{A}^{d}$ gives an anti-linear anti-involution $\omega$ on $\widehat{\mathrm{gl}}_{\infty \mid \infty}$ defined by

$$
C \mapsto C, \quad \sum_{p \in \frac{1}{2} \mathbb{Z}} a_{p} e_{p-k, p} \mapsto \sum_{p \in \frac{1}{2} \mathbb{Z}}(-1)^{[p]+[p-k]} \bar{a}_{p} e_{p, p-k},
$$

for all $\sum_{p \in \frac{1}{2} \mathbb{Z}} a_{p} e_{p-k, p} \in\left(\widehat{\mathrm{gl}}_{\infty \mid \infty}\right)_{k}$ and for all $k \in \frac{1}{2} \mathbb{Z}$. Here $\bar{a}$ denotes the complex conjugate of the complex number $a$. In fact, the realization (5.2), (5.3) of $\widehat{\mathrm{gl}}_{\infty \mid \infty}^{f}$ in 
$\mathscr{A}^{d}$ defines a $*$-superalgebra homomorphism $\Phi:\left(\mathcal{U}\left(\widehat{\mathrm{gl}}_{\infty \mid \infty}^{f}\right), \tilde{\omega}\right) \rightarrow\left(\mathscr{A}^{d}, \omega\right)$, where $\tilde{\omega}$ is the restriction of $\omega$ on $\widehat{\mathrm{gl}}_{\infty \mid \infty}$ to $\widehat{\mathrm{gl}}_{\infty \mid \infty}^{f}$.

Since the Fock space $\mathfrak{F}^{d}$ equipped with the Hermitian form $\langle\cdot \mid \cdot\rangle$ is a unitarizable $\mathscr{A}^{d}$-module, it naturally restricts to a unitarizable module over $\widehat{\mathrm{gl}}_{\infty \mid \infty}^{f}$. Hence $\mathfrak{F}^{d}$ forms a unitarizable $\widehat{\mathrm{gl}}_{\infty \mid \infty}$-module as can be easily seen by examining the action of $\widehat{\mathrm{gl}}_{\infty \mid \infty}$ on $\mathfrak{F}^{d}$. By Theorem 5.1, for each generalized partition $\lambda$ of length $d$, the irreducible $\widehat{\mathrm{gl}}_{\infty \mid \infty}$-module $L\left(\widehat{\mathrm{gl}}_{\infty \mid \infty}, \Lambda(\lambda)\right)$ is unitarizable. We shall show that such modules exhaust all the unitarizable irreducible quasi-finite highest weight $\widehat{\mathrm{gl}}_{\infty \mid \infty}$-modules with respect to the $*$-structure $\omega$.

Theorem 5.6. Let $M$ be an irreducible quasi-finite highest weight $\widehat{\mathrm{gl}}_{\infty \mid \infty}$-module with highest weight $\xi$. Then $M$ is unitarizable with respect to the $*$-structure $\omega$ if and only if $\xi=\Lambda(\lambda)$ for some generalized partition $\lambda$. In other words, $M$ is unitarizable if and only if

$$
\xi=\sum_{s+\frac{1}{2} \leq j \leq r, j \in \frac{1}{2} \mathbb{Z}} \xi_{j} \omega_{j}+d \Lambda_{0}
$$

such that $d \in \mathbb{Z}_{+},-s, r \in \mathbb{N}$ and $\xi_{j} \in \mathbb{Z}$ for all $j$ satisfying the following conditions:

(i) $\xi_{\frac{1}{2}}>\xi_{\frac{3}{2}}>\cdots>\xi_{r-\frac{1}{2}} \geq 0, \xi_{1}>\xi_{2}>\cdots>\xi_{r} \geq 0$, and $\xi_{r-\frac{1}{2}}=0$ if and only if $r=1$ and $\xi_{\frac{1}{2}}=\xi_{1}=0$,

(ii) $0 \geq \xi_{s+\frac{1}{2}}>\xi_{s+\frac{3}{2}}>\cdots>\xi_{-\frac{1}{2}}, 0 \geq \xi_{s+1}>\xi_{s+2}>\cdots>\xi_{0}$, and $\xi_{s+1}=0$ if and only if $s=-1$ and $\xi_{-\frac{1}{2}}=\xi_{0}=0$,

(iii) $\min \left\{\xi_{\frac{1}{2}}, 1\right\}+\xi_{1}-\xi_{0} \leq d$.

Proof. We already know that for any generalized partition $\lambda$, the $\widehat{\mathrm{gl}} \mathrm{\infty}_{\infty}$-module $L\left(\widehat{\mathrm{gl}}_{\infty \mid \infty}, \Lambda(\lambda)\right)$ is unitarizable. Now we show that if $M$ is a unitarizable irreducible quasi-finite highest weight $\widehat{\mathrm{gl}}_{\infty \mid \infty}$-module with the highest weight $\xi$, then $\xi=\Lambda(\lambda)$ for some generalized partition $\lambda$. Let $\langle\cdot \mid \cdot\rangle$ be a positive definite contravariant Hermitian form on $M$ and $v_{\xi}$ a highest weight vector of $M$ such that $\left\langle v_{\xi} \mid v_{\xi}\right\rangle=1$. We put $\xi\left(e_{i i}\right)=\xi_{i}$ for all $i \in \frac{1}{2} \mathbb{Z}$. By Theorem 4.2, there exists $N \in \mathbb{N}$ such that $\xi=\sum_{|j| \leq N, j \in \frac{1}{2} \mathbb{Z}} \xi_{j} \omega_{j}+d \Lambda_{0}$, where $\xi_{j}, d \in \mathbb{C}$. For each $i \in \mathbb{N}$, $\left\{e_{i, i}-e_{i+1, i+1}, e_{i, i+1}, e_{i+1, i}\right\}$ forms a standard basis for the Lie algebra $\operatorname{sl}(2, \mathbb{C})$ and $\omega\left(e_{i, i+1}\right)=e_{i+1, i}$. Unitarizability of $M$ with respect to this subalgebra requires (see, e.g., Theorem 11.7 in [16]) $\xi_{i}-\xi_{i+1}=\xi\left(e_{i, i}-e_{i+1, i+1}\right) \in \mathbb{Z}_{+}$. Since $M$ is a quasi-finite highest weight $\widehat{\mathrm{gl}}_{\infty \mid \infty}$-module, we have $\xi_{i} \in \mathbb{Z}_{+}$for all $i \in \mathbb{N}$ and

$$
\xi_{1} \geq \xi_{2} \geq \cdots \geq \xi_{n} \geq \xi_{n+1}=\xi_{n+2}=\cdots=0 \quad \text { for some } n \in \mathbb{N} \text {. }
$$

Similarly, we have $\xi_{i} \in \mathbb{Z}_{+}$for all $i \in \frac{1}{2}+\mathbb{Z}_{+}$and

$$
\xi_{\frac{1}{2}} \geq \xi_{\frac{3}{2}} \geq \cdots \geq \xi_{r-\frac{1}{2}} \geq \xi_{r+\frac{1}{2}}=\xi_{r+\frac{3}{2}}=\cdots=0 \quad \text { for some } r \in \mathbb{N} \text {. }
$$

Now we are going to show that for each $i \in \frac{1}{2} \mathbb{N}$, either

$$
\xi_{i}>\xi_{i+1} \quad \text { or } \quad \xi_{i}=\xi_{i+\frac{1}{2}}=0 .
$$

It is sufficient to show that $\xi_{i}=\xi_{i+1}$ implies $\xi_{i}=\xi_{i+\frac{1}{2}}=0$. Assume that $\xi_{i}=\xi_{i+1}$. Then $\left\langle e_{i+1, i} v_{\xi} \mid e_{i+1, i} v_{\xi}\right\rangle=\xi_{i}-\xi_{i+1}=0$. Therefore, we have $e_{i+1, i} v_{\xi}=0$. On the 
other hand, we have

$$
\begin{aligned}
0=\left\langle e_{i+\frac{1}{2}, i+1} e_{i+1, i} v_{\xi} \mid e_{i+\frac{1}{2}, i+1} e_{i+1, i} v_{\xi}\right\rangle & =\left\langle e_{i+\frac{1}{2}, i} v_{\xi} \mid e_{i+\frac{1}{2}, i} v_{\xi}\right\rangle \\
& =\xi_{i}+\xi_{i+\frac{1}{2}} .
\end{aligned}
$$

Thus we have $\xi_{i}=\xi_{i+\frac{1}{2}}=0$ since $\xi_{i}, \xi_{i+\frac{1}{2}} \geq 0$. By (5.20), there is $r \in \mathbb{N}$ such that

$$
\begin{aligned}
& \xi_{1}>\xi_{2}>\cdots>\xi_{r} \geq \xi_{r+1}=\xi_{r+2}=\cdots=0, \\
& \xi_{\frac{1}{2}}>\xi_{\frac{3}{2}}>\cdots>\xi_{r-\frac{1}{2}}>\xi_{r+\frac{1}{2}}=\xi_{r+\frac{3}{2}}=\cdots=0,
\end{aligned}
$$

or $\xi_{\frac{1}{2}}=\xi_{1}=0$. By using similar argument as above (note that $\omega\left(e_{i-\frac{1}{2}, i}\right)=-e_{i, i-\frac{1}{2}}$ for all $-i \in \mathbb{Z}_{+}$), we have $-\xi_{i} \in \mathbb{Z}_{+}$for all $-i \in \frac{1}{2} \mathbb{Z}_{+}$, and there is $s \in \mathbb{N}$ such that

$$
\begin{aligned}
& 0=\cdots=\xi_{s-\frac{3}{2}}=\xi_{s-\frac{1}{2}} \geq \xi_{s+\frac{1}{2}}>\xi_{s+\frac{3}{2}}>\cdots>\xi_{-\frac{1}{2}}, \\
& 0=\cdots=\xi_{s-1}=\xi_{s}>\xi_{s+1}>\xi_{s+2}>\cdots>\xi_{0},
\end{aligned}
$$

or $\xi_{-\frac{1}{2}}=\xi_{0}=0$.

Now we choose a large positive integer $n$ such that $\xi\left(e_{n, n}\right)=\xi\left(e_{-n,-n}\right)=0$. Consider the subalgebra $s l(2, \mathbb{C})$ spanned by $\left\{e_{-n,-n}-e_{n, n}+C, e_{-n, n}, e_{n,-n}\right\}$. Note that $\omega\left(e_{-n, n}\right)=e_{n,-n}$. Using the standard trick on unitarizable modules again, we have $d=\xi(C)=\xi\left(e_{-n,-n}-e_{n, n}+C\right) \in \mathbb{Z}_{+}$. Finally, we need to show $\min \left\{\xi_{\frac{1}{2}}, 1\right\}+\xi_{1}-\xi_{0} \leq d$. Since $\left\langle e_{1,0} v_{\xi} \mid e_{1,0} v_{\xi}\right\rangle \geq 0$ and $\left\langle e_{1,0} v_{\xi} \mid e_{1,0} v_{\xi}\right\rangle=\xi_{0}-\xi_{1}+d$, we have $\xi_{0}-\xi_{1}+d \geq 0$. If $\xi_{0}-\xi_{1}+d>0$, the proof of the theorem is completed. Otherwise, we have $\xi_{0}-\xi_{1}+d=0$ and $e_{1,0} v_{\xi}=0$. Therefore, we have

$$
d+\xi_{0}=\xi_{1} \geq 0 .
$$

On the other hand, by using (5.21) and $e_{1,0} v_{\xi}=0$, we have

$$
\begin{aligned}
0=\left\langle e_{\frac{1}{2}, 1} e_{1,0} v_{\xi} \mid e_{\frac{1}{2}, 1} e_{1,0} v_{\xi}\right\rangle & =\left\langle e_{\frac{1}{2}, 0} v_{\xi} \mid e_{\frac{1}{2}, 0} v_{\xi}\right\rangle \\
& =\xi_{0}+\xi_{\frac{1}{2}}+d \\
& =\xi_{\frac{1}{2}}+\xi_{1} .
\end{aligned}
$$

Since $\xi_{\frac{1}{2}} \geq 0$ and $\xi_{1} \geq 0$, we have $\xi_{\frac{1}{2}}=\xi_{1}=0$ and $\min \left\{\xi_{\frac{1}{2}}, 1\right\}+\xi_{1}-\xi_{0}=-\xi_{0} \leq d$ by (5.21) again. This completes the proof of the theorem.

For $\hat{\mathfrak{g}}=\widehat{\mathcal{A}}, \widehat{\mathfrak{C}}$ or $\widehat{\mathcal{D}}$, the restriction of the anti-linear anti-involution $\omega$ on the Lie superalgebra $\widehat{\mathrm{gl}}_{\infty \mid \infty}$ to $\hat{\mathfrak{g}}$ gives an anti-linear anti-involution on $\hat{\mathfrak{g}}$, which will also be denoted by $\omega$. Using similar arguments as in the proof of Theorem [5.6, we can prove the following theorems.

Theorem 5.7. Let $M$ be an irreducible quasi-finite highest weight $\widehat{\mathcal{A}}$-module with highest weight $\xi$. Then $M$ is unitarizable if and only if $\xi=\Lambda^{\widehat{\mathcal{A}}}(\lambda)$ for some generalized partition $\lambda$. In other words, $M$ is unitarizable if and only if

$$
\Lambda^{\widehat{\mathcal{A}}}(\lambda):=\sum_{j \leq r, j \in \frac{1}{2} \mathbb{N}} \xi_{j}^{+} \omega_{j}-\sum_{j \geq-s, j \in-\frac{1}{2} \mathbb{N}} \xi_{-j}^{-} \omega_{j}+d \Lambda_{0}
$$

such that $d \in \mathbb{Z}_{+}, s, r \in \mathbb{N}$ and $\xi_{j} \in \mathbb{Z}$ for all $j$ satisfying the following conditions:

(i) $\xi_{\frac{1}{2}}^{+}>\xi_{\frac{3}{2}}^{+}>\cdots>\xi_{r-\frac{1}{2}}^{+} \geq 0, \xi_{1}^{+}>\xi_{2}^{+}>\cdots>\xi_{r}^{+} \geq 0$, and $\xi_{r-\frac{1}{2}}^{+}=0$ if and only if $r=1$ and $\xi_{\frac{1}{2}}^{+}=\xi_{1}^{+}=0$, 
(ii) $\xi_{\frac{1}{2}}^{-}>\xi_{\frac{3}{2}}^{-}>\cdots>\xi_{s-\frac{1}{2}}^{-} \geq 0, \xi_{1}^{-}>\xi_{2}^{-}>\cdots>\xi_{s}^{-} \geq 0$, and $\xi_{s-\frac{1}{2}}^{-}=0$ if and only if $s=1$ and $\xi_{\frac{1}{2}}^{-}=\xi_{1}^{-}=0$,

(iii) $\min \left\{\xi_{\frac{1}{2}}^{+}, 1\right\}+\min \left\{\xi_{\frac{1}{2}}^{-}, 1\right\}+\xi_{1}^{+}+\xi_{1}^{-} \leq d$.

Theorem 5.8. Let $M$ be an irreducible quasi-finite highest weight $\widehat{\mathrm{C}}$-module with highest weight $\xi$. Then $M$ is unitarizable if and only if $\xi=\Lambda^{\widehat{\mathrm{e}}}(\lambda)$ for some partition $\lambda$ of length $d$. In other words, $M$ is unitarizable if and only if

$$
\Lambda^{\widehat{\mathrm{e}}}(\lambda):=\sum_{j \leq r, j \in \frac{1}{2} \mathbb{N}} \xi_{j} \omega_{j}+d \Lambda_{0}
$$

such that $d \in \mathbb{Z}_{+}, r \in \mathbb{N}$ and $\xi_{j} \in \mathbb{Z}$ for all $j$ satisfying the following conditions:

(i) $\xi_{\frac{1}{2}}>\xi_{1 \frac{1}{2}}>\cdots>\xi_{r-\frac{1}{2}} \geq 0, \xi_{1}>\xi_{2}>\cdots>\xi_{r} \geq 0$, and $\xi_{r-\frac{1}{2}}=0$ if and only if $r=1$ and $\xi_{\frac{1}{2}}=\xi_{1}=0$,

(ii) $\min \left\{\xi_{\frac{1}{2}}, 1\right\}+\xi_{1} \leq d$.

Since the Fock space realizations of $\widehat{\mathcal{D}}$ differ considerably from those for the other algebras, we treat unitarizable $\widehat{\mathcal{D}}$-modules in some detail. Introduce an anti-linear anti-involution $\omega$ on $\mathscr{A}_{0}^{d+\frac{1}{2}}$ in the following way. It is defined by (5.18) and (5.19) on all the $\widetilde{\psi}_{j}^{ \pm, i}$ and $\gamma_{j}^{ \pm, i}$, and

$$
\omega\left(\phi_{i}\right)=\phi_{-i}, \quad \text { for all } i ; \quad \omega\left(\chi_{r}\right)=\chi_{-r}, \quad \text { for all } r .
$$

The Fock space $\mathfrak{F}_{0}^{d+\frac{1}{2}}$ admits a positive definite contravariant Hermitian form with respect to this $*$-structure of $\mathscr{A}_{0}^{d+\frac{1}{2}}$.

It is important to observe that the restrictions of the $*$-structures of $\mathscr{A}_{0}^{d+\frac{1}{2}}$ and $\widehat{\mathrm{gl}}_{\infty \mid \infty}$ to $\widehat{\mathcal{D}}^{f}$ coincide. Thus all the irreducible $\widehat{\mathcal{D}}$-modules appearing in Theorem 5.5 are unitarizable with respect to the the anti-linear anti-involution $\omega$.

Theorem 5.9. Let $M$ be an irreducible quasi-finite highest weight $\widehat{\mathcal{D}}$-module with highest weight $\xi$. Then $M$ is unitarizable if and only if there exists a non-negative integer or half integer $k$ such that $\xi=\Lambda^{\widehat{\mathcal{D}}}(\lambda)$ for some partition $\lambda$ of length $2 k$ with $\lambda_{1}^{\prime}+\lambda_{2}^{\prime} \leq 2 k$. In other words, $M$ is unitarizable if and only if

$$
\Lambda^{\widehat{\mathcal{D}}}(\lambda):=\sum_{j \leq r, j \in \frac{1}{2} \mathbb{N}} \xi_{j} \omega_{j}+k \Lambda_{0}
$$

such that $k \in \frac{1}{2} \mathbb{Z}_{+}, r \in \mathbb{N}$ and $\xi_{j} \in \mathbb{Z}$ for all $j$ satisfying the following conditions:

(i) $\xi_{\frac{1}{2}}>\xi_{\frac{3}{2}}>\cdots>\xi_{r-\frac{1}{2}} \geq 0, \xi_{1}>\xi_{2}>\cdots>\xi_{r} \geq 0$, and $\xi_{r-\frac{1}{2}}=0$ if and only if $r=1$ and $\xi_{\frac{1}{2}}=\xi_{1}=0$,

(ii) $\xi_{1}+\xi_{2}+l_{1,2}\left(\xi_{\frac{1}{2}}\right)+\min \left\{\xi_{\frac{3}{2}}, 1\right\} \leq 2 k$,

where $l_{1,2}$ is a function from non-negative integers to itself with $l_{1,2}(0)=0, l_{1,2}(1)=$ 1 and $l_{1,2}(x)=2$ if $x \geq 2$.

Proof. We only need to prove the "only if" part of the theorem. Let $\langle\cdot \mid \cdot\rangle$ be a positive definite contravariant Hermitian form on $M$ and $v_{\xi}$ a highest weight vector of $M$ such that $\left\langle v_{\xi} \mid v_{\xi}\right\rangle=1$. We put $\xi\left(\tilde{e}_{i, i}\right)=\xi_{i}$ for all $i \in \frac{1}{2} \mathbb{N}$. By Theorem 4.2. there exists $r \in \mathbb{N}$ such that $\xi=\sum_{j \leq r, j \in \frac{1}{2} \mathbb{N}} \xi_{j} \omega_{j}+k \Lambda_{0}$, where $\xi_{j}, k \in \mathbb{C}$. By using 
similar arguments as in the proof of Theorem [5.6, we can show that $\xi_{i} \in \mathbb{Z}_{+}$for $i=\frac{1}{2}, 1, \cdots, r-\frac{1}{2}, r$, and

$$
\xi_{\frac{1}{2}}>\xi_{1 \frac{1}{2}}>\cdots>\xi_{r-\frac{1}{2}} \geq 0, \quad \xi_{1}>\xi_{2}>\cdots>\xi_{r} \geq 0 .
$$

Moreover, $\xi_{r-\frac{1}{2}}=0$ if and only if $r=1$ and $\xi_{\frac{1}{2}}=\xi_{1}=0$.

Now we choose a large positive integer $n$ such that $\xi\left(\tilde{e}_{n, n}\right)=\xi\left(\tilde{e}_{n+1, n+1}\right)=0$. Consider the subalgebra $\operatorname{sl}(2, \mathbb{C})$ with the standard basis $\left\{2 C-\tilde{e}_{n, n}-\tilde{e}_{n+1, n+1}\right.$, $\left.\tilde{e}_{-n-1, n}, \tilde{e}_{n,-n-1}\right\}$. Note that $\omega\left(2 C-\tilde{e}_{n, n}-\tilde{e}_{n+1, n+1}\right)=2 C-\tilde{e}_{n, n}-\tilde{e}_{n+1, n+1}$, $\omega\left(\tilde{e}_{n,-n-1}\right)=\tilde{e}_{-n-1, n}$ and $\omega\left(-\tilde{e}_{-n-1, n}\right)=-\tilde{e}_{n,-n-1}$. Unitarizability with respect to this $s l_{2}$ subalgebra requires $2 k=\xi(2 C)=\xi\left(2 C-\tilde{e}_{n, n}-\tilde{e}_{n+1, n+1}\right) \in \mathbb{Z}_{+}$. Hence we have $k \in \frac{1}{2} \mathbb{Z}_{+}$.

Finally, we need to show $\xi_{1}+\xi_{2}+l_{1,2}\left(\xi_{\frac{1}{2}}\right)+\min \left\{\xi_{\frac{3}{2}}, 1\right\} \leq 2 k$. We may assume that $\xi_{\frac{1}{2}}>0$. Otherwise, we have $\xi_{1}+\xi_{2}+l_{1,2}\left(\xi_{\frac{1}{2}}\right)+\min \left\{\xi_{\frac{3}{2}}, 1\right\}=0 \leq 2 k$. Direct computations show that $\left\langle\tilde{e}_{1, \frac{1}{2}} v_{\xi} \mid \tilde{e}_{1, \frac{1}{2}} v_{\xi}\right\rangle=\xi_{\frac{1}{2}}+\xi_{1}>0$ and

$$
\begin{aligned}
0 \leq\left\|\tilde{e}_{2,-1} \tilde{e}_{1, \frac{1}{2}} v_{\xi}\right\|^{2} & =\left\langle\tilde{e}_{1, \frac{1}{2}} v_{\xi} \mid\left(2 C-\tilde{e}_{1,1}-\tilde{e}_{2,2}\right) \tilde{e}_{1, \frac{1}{2}} v_{\xi}\right\rangle \\
& =\left(2 k-\xi_{1}-\xi_{2}-1\right)\left(\xi_{\frac{1}{2}}+\xi_{1}\right) .
\end{aligned}
$$

Thus we have $2 k-\xi_{1}-\xi_{2}-1 \geq 0$ since $\xi_{\frac{1}{2}}+\xi_{1}>0$. When $\xi_{\frac{1}{2}}=1$, we have $\xi_{\frac{3}{2}}=\xi_{2}=0$. Thus $\xi_{1}+\xi_{2}+l_{1,2}\left(\xi_{\frac{1}{2}}\right)+\min \left\{\xi_{\frac{3}{2}}, 1\right\}=\xi_{1}+\xi_{2}+1+0 \leq 2 k$. Now we assume that $\xi_{\frac{1}{2}} \geq 2$. We compute

$$
\begin{aligned}
\left\|\tilde{e}_{-\frac{1}{2}, 1} \tilde{e}_{2,-1} \tilde{e}_{1, \frac{1}{2}} v_{\xi}\right\|^{2} & =\left\|\tilde{e}_{2, \frac{1}{2}} \tilde{e}_{1, \frac{1}{2}} v_{\xi}\right\|^{2} \\
& =\left\langle\tilde{e}_{1, \frac{1}{2}} v_{\xi} \mid\left(\tilde{e}_{\frac{1}{2}, \frac{1}{2}}+\tilde{e}_{2,2}\right) \tilde{e}_{1, \frac{1}{2}} v_{\xi}\right\rangle \\
& =\left(\xi_{\frac{1}{2}}+\xi_{2}-1\right)\left(\xi_{\frac{1}{2}}+\xi_{1}\right)>0 .
\end{aligned}
$$

Thus $\left\|\tilde{e}_{2,-1} \tilde{e}_{1, \frac{1}{2}} v_{\xi}\right\|^{2}>0$ and hence we have $\xi_{1}+\xi_{2}+l_{1,2}\left(\xi_{\frac{1}{2}}\right)+\min \left\{\xi_{\frac{3}{2}}, 1\right\}=$ $\xi_{1}+\xi_{2}+2 \leq 2 k$ for $\xi_{\frac{1}{2}} \geq 2$ and $\xi_{\frac{3}{2}}=0$ by using (5.23). Eventually we assume that $\xi_{\frac{1}{2}} \geq 2$ and $\xi_{\frac{3}{2}}>0$. Then

$$
\begin{aligned}
\left\|\tilde{e}_{-\frac{1}{2}, 1} \tilde{e}_{2,-1} \tilde{e}_{2, \frac{3}{2}} \tilde{e}_{1, \frac{1}{2}} v_{\xi}\right\|^{2} & =\left\|\tilde{e}_{2, \frac{1}{2}} \tilde{e}_{2, \frac{3}{2}} \tilde{e}_{1, \frac{1}{2}} v_{\xi}\right\|^{2} \\
& =\left(\xi_{\frac{1}{2}}+\xi_{2}\right)\left\|\tilde{e}_{2, \frac{3}{2}} \tilde{e}_{1, \frac{1}{2}} v_{\xi}\right\|^{2} \\
& =\left(\xi_{\frac{1}{2}}+\xi_{2}\right)\left(\xi_{\frac{1}{2}}+\xi_{1}\right)\left(\xi_{\frac{1}{2}}+\xi_{2}\right)>0 .
\end{aligned}
$$

Therefore, we have $\tilde{e}_{2,-1} \tilde{e}_{2, \frac{3}{2}} \tilde{e}_{1, \frac{1}{2}} v_{\xi} \neq 0$ and

$$
\begin{aligned}
0<\left\|\tilde{e}_{2,-1} \tilde{e}_{2, \frac{3}{2}} \tilde{e}_{1, \frac{1}{2}} v_{\xi}\right\|^{2} & =\left\langle\tilde{e}_{2, \frac{3}{2}} \tilde{e}_{1, \frac{1}{2}} v_{\xi} \mid \tilde{e}_{-1,2} \tilde{e}_{2,-1} \tilde{e}_{2, \frac{3}{2}} \tilde{e}_{1, \frac{1}{2}} v_{\xi}\right\rangle \\
& =\left(2 k-\xi_{\frac{1}{2}}-\xi_{2}-2\right)\left\|\tilde{e}_{2, \frac{3}{2}} \tilde{e}_{1, \frac{1}{2}} v_{\xi}\right\|^{2} \\
& =\left(2 k-\xi_{\frac{1}{2}}-\xi_{2}-2\right)\left(\xi_{\frac{1}{2}}+\xi_{1}\right)\left(\xi_{\frac{1}{2}}+\xi_{2}\right) .
\end{aligned}
$$

Therefore, we have $\xi_{\frac{1}{2}}+\xi_{2}+3 \leq 2 k$. Hence $\xi_{1}+\xi_{2}+l_{1,2}\left(\xi_{\frac{1}{2}}\right)+\min \left\{\xi_{\frac{3}{2}}, 1\right\}=$ $\xi_{\frac{1}{2}}+\xi_{2}+3 \leq 2 k$ and the proof is completed.

Recall that in 24] Wang showed that there is a $\widehat{\mathrm{gl}}_{\infty} \times g l_{d}$ duality on the subspace of the Fock space $\mathfrak{F}^{d}$ generated by the fermionic operators. By modifying the arguments in the proof of Theorem [5.6 we can show the following theorem. 
Theorem 5.10. Let $M$ be an irreducible quasi-finite highest weight $\widehat{\mathrm{gl}}_{\infty}$-module with the highest weight $\xi$. Then $M$ is unitarizable if and only if

$$
\xi=\sum_{s \leq j \leq r} \xi_{j} \omega_{j}+d \Lambda_{0}
$$

such that $d \in \mathbb{Z}_{+}, r \in \mathbb{N},-s \in \mathbb{Z}_{+}$and $\xi_{j} \in \mathbb{Z}$ for all $j$ satisfying the following conditions:

(i) $\xi_{1}>\xi_{2}>\cdots>\xi_{r} \geq 0$,

(ii) $0 \geq \xi_{s}>\xi_{s+1}>\cdots>\xi_{0}$,

(iii) $\xi_{1}-\xi_{0} \leq d$.

\section{Character formulas for unitarizable IRreducible modules}

In this section we derive explicit formulae for the formal characters of the unitarizable quasi-finite irreducible highest weight modules over the infinite rank Lie algebras $\widehat{\mathcal{C}}$ and $\widehat{\mathcal{D}}$. The method employed here is a generalization of that developed in [3, 8, 4, which makes essential use of Howe dualities. We mention that the character formulae for the unitarizable irreducible modules over $\left.\widehat{\mathrm{gl}}_{\infty}\right|_{\infty}$ (and hence $\widehat{\mathcal{A}})$ were obtained in 3 .

6.1. Character formula for $\widehat{\mathcal{C}}$. The main result of this subsection is Theorem 6.1 which gives the character formula for the unitarizable quasi-finite irreducible highest weight $\widehat{\mathcal{C}}$-modules. In order to establish the result, we need some basic facts on characters of the symplectic group (see [10, 13, 14]), which we now recall.

When we deal with characters of $\mathfrak{s p}(2 m)$-modules, we let $z_{i}:=e^{\epsilon_{i}}$ where $\epsilon_{i} \in \mathfrak{h}^{*}$ such that $\epsilon_{i}\left(h_{j}\right):=\delta_{i j}$ (see Section [5.3). We define $\tilde{h}_{i}:=-h_{m-i+1}$ and $x_{i}:=$ $e^{-\epsilon_{m-i+1}}$ for $i=1,2, \cdots, m$. That is, $x_{i}=z_{m-i+1}^{-1}$. The definitions of $x_{i}$ 's and $\tilde{h}_{i}$ 's are somewhat non-standard, but they allow us to deal with only polynomials instead of Laurent polynomials when considering characters of certain representations of $\mathfrak{s p}(2 m)$. When we deal with characters of $S p(2 d)$-modules, we put $z_{i}=e^{\epsilon_{i}}$ where $\epsilon_{i} \in \mathfrak{h}^{*}$ such that $\epsilon_{i}\left(h_{j}\right)=\epsilon_{i}\left(e_{j j}-e_{d+j, d+j}\right)=\delta_{i j}$. When dealing with characters of $\widehat{\mathrm{C}}$-modules, for $j \in \mathbb{N}, r \in \frac{1}{2}+\mathbb{Z}_{+}$, we let $x_{j}:=e^{\omega_{j}}$ and $y_{r}:=e^{\omega_{r}}$, where $\omega_{s}$ are the respective fundamental weights of $\widehat{e}$ introduced in Section 4.2.

For each sequence of complex numbers $\lambda=\left(\lambda_{1}, \cdots, \lambda_{m}\right)$, we let $W_{\mathfrak{s p}(2 m)}^{\lambda}:=$ $V_{\mathfrak{s p}(2 m)}^{\lambda^{*}}$ where $\lambda^{*}=\left(-\lambda_{m}, \cdots,-\lambda_{1}\right)$. Note that $\lambda^{*}\left(\tilde{h}_{i}\right)=\lambda_{i}$ for $i=1, \cdots, m$. The module $W_{\mathfrak{s p}(2 m)}^{\lambda}$ is finite-dimensional if and only if $\lambda_{1} \geq \lambda_{2} \geq \cdots \geq \lambda_{m}$ and $\lambda_{i} \in-\mathbb{Z}_{+}$for $i=1, \cdots, m$.

For each partition $\lambda$ of length $d$ and each decreasing sequence of non-positive integers $\nu$ of length $m$, we write

$$
\chi_{S p(2 d)}^{\lambda}(\mathbf{z})=\chi_{S p(2 d)}^{\lambda}\left(z_{1}, \cdots, z_{d}\right), \quad \tilde{\chi}_{\mathfrak{s p}(2 m)}^{\nu}(\mathbf{x})=\tilde{\chi}_{\mathfrak{s p}(2 m)}^{\nu}\left(x_{1}, \cdots, x_{m}\right)
$$

for the characters of the $S p(2 d)$-module $V_{S p(2 d)}^{\lambda}$ and the $\mathfrak{s p}(2 m)$-module $W_{\mathfrak{s p}(2 m)}^{\nu}$, to stress their dependence on the variables $z_{1}, \cdots, z_{d}$ and $x_{1}, \cdots, x_{m}$, respectively. It is clear that $\chi_{S p(2 m)}^{\lambda}(\mathbf{z})=\tilde{\chi}_{\mathfrak{s p}(2 m)}^{\lambda^{*}}\left(z_{1}^{-1}, \cdots, z_{m}^{-1}\right)$ for any partition $\lambda$ of length $m$ since $\chi_{\mathfrak{s p}(2 m)}^{\lambda}\left(z_{1}, \cdots, z_{m}\right)=\chi_{S p(2 m)}^{\lambda^{*}}\left(z_{1}^{-1}, \cdots, z_{m}^{-1}\right)$. 
By the $(S p(2 d), \mathfrak{s p}(2 m))$-duality on the exterior algebra $\Lambda\left(\mathbb{C}^{2 d} \otimes \mathbb{C}^{m}\right)$ with $m \geq d$ ([13], [14], also see [8]), we have the following identity:

$$
\left(x_{1} \cdots x_{m}\right)^{-d} \prod_{i=1}^{d} \prod_{j=1}^{m}\left(1+x_{j} z_{i}\right)\left(1+x_{j} z_{i}^{-1}\right)=\sum_{\lambda} \chi_{S p(2 d)}^{\lambda}(\mathbf{z}) \tilde{\chi}_{\mathfrak{s p}(2 m)}^{\lambda^{\prime}-d\left(1^{m}\right)}(\mathbf{x}) .
$$

Here $\lambda$ runs over all partitions of length $d$ with $\lambda_{1} \leq m$, and for any $k \in \mathbb{N},\left(1^{k}\right)$ stands for the $k$-tuple $(1,1, \cdots, 1)$. Note that the partition $\lambda^{\prime}$ is considered as a partition of length $m$ and $\lambda^{\prime}-d\left(1^{m}\right)$ is a decreasing sequence of non-positive integers of length $m$.

We let

$$
\begin{aligned}
E_{r}^{\prime}\left(x_{1}, \cdots, x_{m}, x_{1}^{-1}, \cdots, x_{m}^{-1}\right)= & E_{r}\left(x_{1}, \cdots, x_{m}, x_{1}^{-1}, \cdots, x_{m}^{-1}\right) \\
& -E_{r-2}\left(x_{1}, \cdots, x_{m}, x_{1}^{-1}, \cdots, x_{m}^{-1}\right),
\end{aligned}
$$

where $E_{r}$ is the $r$-th elementary symmetric polynomial for $r \geq 0$ and $E_{r}=0$ for $r<0$. For any partition $\mu$ of length $l$ (which is an arbitrary positive integer), we define

$$
\left|E_{\mu}^{\prime}\right|=\left|E_{\mu}^{\prime}\left(x_{1}, \cdots, x_{m}, x_{1}^{-1}, \cdots, x_{m}^{-1}\right)\right|,
$$

the determinant of the $l \times l$ matrix whose $i$-th row is

$$
\left(E_{\mu_{i}-i+1}^{\prime}, E_{\mu_{i}-i+2}^{\prime}+E_{\mu_{i}-i}^{\prime} E_{\mu_{i}-i+3}^{\prime}+E_{\mu_{i}-i-1}^{\prime} \cdots E_{\mu_{i}-i+l}^{\prime}+E_{\mu_{i}-i-l+2}^{\prime}\right) .
$$

Then the character of the irreducible $\mathfrak{s p}(2 m)$-module $V_{\mathfrak{s p}(2 m)}^{\lambda}$ is given by $\left|E_{\lambda^{\prime}}^{\prime}\right|=$ $\left|E_{\lambda^{\prime}}^{\prime}\left(x_{1}, \cdots, x_{m}, x_{1}^{-1}, \cdots, x_{m}^{-1}\right)\right|$, where $\lambda$ is any partition of length $m$ [10].

From the above result it is not very difficult to work out that the character $\tilde{\chi}_{\mathfrak{s p}(2 m)}^{\lambda^{\prime}-d\left(1^{m}\right)}(\mathbf{x})$ of the finite-dimensional irreducible $\mathfrak{s p}(2 m)$-module $W_{\mathfrak{s p}(2 m)}^{\lambda^{\prime}-d\left(1^{m}\right)}$ for every partition $\lambda$ of length $d$ with $\lambda_{1} \leq m$ is given by the determinant of the $d \times d$ matrix whose $i$-th row is

$$
\begin{aligned}
& \left(E_{m-\lambda_{d-i+1}-i+1}^{\prime} \quad E_{m-\lambda_{d-i+1}-i+2}^{\prime}+E_{m-\lambda_{d-i+1}-i}^{\prime}\right. \\
& \left.E_{m-\lambda_{d-i+1}-i+3}^{\prime}+E_{m-\lambda_{d-i+1}-i-1}^{\prime} \cdots E_{m-\lambda_{d-i+1}-i+d}^{\prime}+E_{m-\lambda_{d-i+1}-i-d+2}^{\prime}\right) .
\end{aligned}
$$

On the other hand, for each $r \in \mathbb{Z}$,

$$
\begin{aligned}
& E_{m-r}\left(x_{1}, \cdots, x_{m}, x_{1}^{-1}, \cdots, x_{m}^{-1}\right) \\
= & \sum_{i=0}^{m-r} E_{i}\left(x_{1}, \cdots, x_{m}\right) E_{m-r-i}\left(x_{1}^{-1}, \cdots, x_{m}^{-1}\right) \\
= & \left(x_{1} \cdots x_{m}\right)^{-1} \sum_{i=0}^{m-r} E_{i}\left(x_{1}, \cdots, x_{m}\right) E_{r+i}\left(x_{1}, \cdots, x_{m}\right) \\
= & \left(x_{1} \cdots x_{m}\right)^{-1} \sum_{i=0}^{\infty} E_{i}\left(x_{1}, \cdots, x_{m}\right) E_{r+i}\left(x_{1}, \cdots, x_{m}\right) .
\end{aligned}
$$

For $r \in \mathbb{Z}$, we define $\widetilde{E}_{r}^{\prime}:=\widetilde{E}_{r}-\widetilde{E}_{r-2}$ with

$$
\widetilde{E}_{r}:=\sum_{i=0}^{\infty} E_{i}\left(x_{1}, \cdots, x_{m}\right) E_{r+i}\left(x_{1}, \cdots, x_{m}\right) .
$$

Then we have

$$
E_{m-r}^{\prime}\left(x_{1}, \cdots, x_{m}, x_{1}^{-1}, \cdots, x_{m}^{-1}\right)=\left(x_{1} \cdots x_{m}\right)^{-1} \widetilde{E}_{r}^{\prime} .
$$


Hence for each partition $\lambda$ of length $d,\left(x_{1} \cdots x_{m}\right)^{d} \tilde{\chi}_{\mathfrak{s p}(2 m)}^{\lambda^{\prime}-d\left(1^{m}\right)}(\mathbf{x})$ equals the determinant of the $d \times d$ matrix whose $i$-th row is

$$
\begin{aligned}
\left(\begin{array}{lll}
\widetilde{E}_{\lambda_{d-i+1}+i-1}^{\prime} & \widetilde{E}_{\lambda_{d-i+1}+i-2}^{\prime}+\widetilde{E}_{\lambda_{d-i+1}+i}^{\prime} & \widetilde{E}_{\lambda_{d-i+1}+i-3}^{\prime}+\widetilde{E}_{\lambda_{d-i+1}+i+1}^{\prime} \\
\cdots & \widetilde{E}_{\lambda_{d-i+1}+i-d}^{\prime}+\widetilde{E}_{\lambda_{d-i+1}+i+d-2}^{\prime}
\end{array}\right) .
\end{aligned}
$$

For each partition $\lambda$ of length $d$ satisfying $\lambda_{1} \leq m$ and $d \leq m$, the symplectic Schur polynomial of weight $d$ of $m$ variables, denoted by

$$
S_{\lambda}^{\mathfrak{s p}, d}(\mathbf{x})=S_{\lambda}^{\mathfrak{s p}, d}\left(x_{1}, \cdots, x_{m}\right),
$$

is defined by the determinant of the $d \times d$ matrix whose $i$-th row is

$$
\begin{aligned}
\left(\begin{array}{lll}
\widetilde{E}_{\lambda_{d-i+1}+i-1}^{\prime} & \widetilde{E}_{\lambda_{d-i+1}+i-2}^{\prime}+\widetilde{E}_{\lambda_{d-i+1}+i}^{\prime} & \widetilde{E}_{\lambda_{d-i+1}+i-3}^{\prime}+\widetilde{E}_{\lambda_{d-i+1}+i+1}^{\prime} \\
\cdots & \widetilde{E}_{\lambda_{d-i+1}+i-d}^{\prime}+\widetilde{E}_{\lambda_{d-i+1}+i+d-2}^{\prime}
\end{array}\right) .
\end{aligned}
$$

Thus we have

$$
\tilde{\chi}_{\mathfrak{s p}(2 m)}^{\lambda^{\prime}-d\left(1^{m}\right)}\left(x_{1}, \cdots, x_{m}\right)=\left(x_{1} \cdots x_{m}\right)^{-d} S_{\lambda}^{\mathfrak{s p}, d}\left(x_{1}, \cdots, x_{m}\right) .
$$

Hence we can rewrite the combinatorial formula (6.1) as follows:

$$
\prod_{i=1}^{d} \prod_{j=1}^{m}\left(1+x_{j} z_{i}\right)\left(1+x_{j} z_{i}^{-1}\right)=\sum_{\lambda} \chi_{S p(2 d)}^{\lambda}(\mathbf{z}) S_{\lambda}^{\mathfrak{s p}, d}(\mathbf{x}) .
$$

Here $\lambda$ runs over all partitions of length $d$ with $\lambda_{1} \leq m$.

Now, for every partition $\lambda$ of length $d$, we still use

$$
S_{\lambda}^{\mathfrak{s}, d}(\mathbf{x})=S_{\lambda}^{\mathfrak{s p}, d}\left(x_{1}, \cdots, x_{m}, \cdots\right)
$$

to denote the symplectic Schur function of weight $d$ of infinitely many variables, which is the determinant of the $d \times d$ matrix whose $i$-th row is

$$
\begin{aligned}
& \left(\tilde{e}_{\lambda_{d-i+1}+i-1}^{\prime} \quad \tilde{e}_{\lambda_{d-i+1}+i-2}^{\prime}+\tilde{e}_{\lambda_{d-i+1}+i}^{\prime} \quad \tilde{e}_{\lambda_{d-i+1}+i-3}^{\prime}+\tilde{e}_{\lambda_{d-i+1}+i+1}^{\prime}\right. \\
& \left.\cdots \tilde{e}_{\lambda_{d-i+1}+i-d}^{\prime}+\tilde{e}_{\lambda_{d-i+1}+i+d-2}^{\prime}\right),
\end{aligned}
$$

where $\tilde{e}_{r}^{\prime}=\tilde{e}_{r}-\tilde{e}_{r-2}$ and $\tilde{e}_{r}=\sum_{i=0}^{\infty} e_{i}\left(x_{1}, x_{2}, \cdots\right) e_{r+i}\left(x_{1}, x_{2}, \cdots\right)$. Hereafter, $e_{i}\left(x_{1}, x_{2}, \cdots\right)$ stands for the i-th elementary symmetric function of infinitely many variables. Therefore, the symplectic Schur function $S_{\lambda}^{\mathfrak{s p}, d}\left(x_{1}, x_{2}, \cdots\right)$ is the inverse limit of the symplectic Schur polynomials $S_{\lambda}^{\mathfrak{s p}, d}\left(x_{1}, \cdots, x_{m}\right)$, and we thus have $S_{\lambda}^{\mathfrak{s p}, d}\left(x_{1}, \cdots, x_{m}\right)=S_{\lambda}^{\mathfrak{s p}, d}\left(x_{1}, \cdots, x_{m}, 0,0, \cdots\right)$.

For any given partition $\lambda$ of length $d$, we let

$$
D S_{\lambda}^{\mathfrak{s p}, d}(\mathbf{x})=D S_{\lambda}^{\mathfrak{s p}, d}\left(x_{1}, x_{2}, \cdots\right)
$$

denote the skew symplectic Schur function of weight $d$ of infinitely many variables, which is defined by the determinant of the $d \times d$ matrix whose $i$-th row is

$$
\begin{aligned}
& \left(\begin{array}{lll}
\tilde{H}_{\lambda_{d-i+1}+i-1}^{\prime} & \tilde{H}_{\lambda_{d-i+1}+i-2}^{\prime}+\tilde{H}_{\lambda_{d-i+1}+i}^{\prime} & \tilde{H}_{\lambda_{d-i+1}+i-3}^{\prime}+\tilde{H}_{\lambda_{d-i+1}+i+1}^{\prime}
\end{array}\right. \\
& \left.\cdots \quad \tilde{H}_{\lambda_{d-i+1}+i-d}^{\prime}+\tilde{H}_{\lambda_{d-i+1}+i+d-2}^{\prime}\right),
\end{aligned}
$$

where $\tilde{H}_{r}^{\prime}:=\tilde{H}_{r}-\tilde{H}_{r-2}$ and

$$
\tilde{H}_{r}:=\sum_{i=0}^{\infty} H_{i}\left(x_{1}, x_{2}, \cdots\right) H_{r+i}\left(x_{1}, x_{2}, \cdots\right) .
$$


Here $H_{i}\left(x_{1}, x_{2}, \cdots\right)$ are the complete symmetric functions of infinitely many variables. Note that $\left(x_{1} \cdots x_{m}\right)^{-d} D S_{\lambda}^{\mathfrak{s p}, d}\left(x_{1}^{-1}, \cdots, x_{m}^{-1}, 0,0, \cdots\right)$ is the character of some infinite dimensional unitarizable module over the Lie algebra $\mathfrak{s o}(2 \mathrm{~m})$.

Corresponding to each partition $\lambda$ of length $d$, we define the symplectic hook Schur function (in analogy with hook Schur functions (see [1] and [3])),

$$
H S_{\lambda}^{\mathfrak{s} \mathfrak{p}, d}(\mathbf{x}, \mathbf{y})=S_{\lambda}^{\mathfrak{s}, d}\left(x_{1}, x_{2}, \cdots, y_{\frac{1}{2}}, y_{\frac{3}{2}}, \cdots\right)
$$

of weight $d$ in infinitely many variables by

$$
H S_{\lambda}^{\mathfrak{s p}, d}(\mathbf{x}, \mathbf{y}):=\sigma\left(S_{\lambda}^{\mathfrak{s p}, d}(\mathbf{x}, \mathbf{y})\right),
$$

where $\sigma$ is the involution of the ring of symmetric functions (see for example [19]), which sends the elementary symmetric functions of $y_{j}$ 's to the complete symmetric functions of $y_{j}$ 's. Recall that the hook Schur function (cf. [3])

$$
H S_{\lambda}(\mathbf{x}, \mathbf{y})=\sigma\left(S_{\lambda}(\mathbf{x}, \mathbf{y})\right)
$$

is a symmetric function of the variables $\mathbf{x}$ and the variables $\mathbf{y}$ separably where $\lambda$ is a partition. Hereafter, $S_{\lambda}$ stands for the Schur function associated with the partition $\lambda$. Then we have

$$
H S_{\left(1^{d}\right)}(\mathbf{x}, \mathbf{y})=\sigma\left(e_{d}(\mathbf{x}, \mathbf{y})\right)=\sum_{i=0}^{d} e_{i}(\mathbf{x}) e_{d-i}(\mathbf{y}) .
$$

The symplectic hook Schur function can be written in terms of hook Schur functions as follows.

Proposition 6.1. Let $\mathbf{x}=\left\{x_{1}, x_{2}, \cdots\right\}$ and $\mathbf{y}=\left\{y_{1}, y_{2}, \cdots\right\}$ be two infinite sets of variables. For each partition $\lambda$ of length $d$, the symplectic hook Schur function $H S_{\lambda}^{\mathfrak{s p}, d}(\mathbf{x}, \mathbf{y})$ of weight $d$ equals the determinant of the following $d \times d$ matrix whose $i$-th row is

$$
\begin{aligned}
& \left(\tilde{f}_{\lambda_{d-i+1}+i-1}^{\prime} \quad \tilde{f}_{\lambda_{d-i+1}+i-2}^{\prime}+\tilde{f}_{\lambda_{d-i+1}+i}^{\prime} \quad \tilde{f}_{\lambda_{d-i+1}+i-3}^{\prime}+\tilde{f}_{\lambda_{d-i+1}+i+1}^{\prime}\right. \\
& \left.\cdots \quad \tilde{f}_{\lambda_{d-i+1}+i-d}^{\prime}+\tilde{f}_{\lambda_{d-i+1}+i+d-2}^{\prime}\right),
\end{aligned}
$$

where $\tilde{f}_{r}^{\prime}=\tilde{f}_{r}-\tilde{f}_{r-2}$ and $\tilde{f}_{r}=\sum_{i=0}^{\infty} H S_{\left(1^{i}\right)}(\mathbf{x}, \mathbf{y}) H S_{\left(1^{r+i}\right)}(\mathbf{x}, \mathbf{y})$.

Proof. Let $\sigma$ denote the involution of the ring of symmetric functions, which sends the elementary symmetric functions of variables $\mathbf{y}$ to the complete symmetric functions of variables $\mathbf{y}$. The proposition follows by applying the involution $\sigma$ to the determinant of the following $d \times d$ matrix whose $i$-th row is

$$
\begin{aligned}
& \left(\tilde{e}_{\lambda_{d-i+1}+i-1}^{\prime} \quad \tilde{e}_{\lambda_{d-i+1}+i-2}^{\prime}+\tilde{e}_{\lambda_{d-i+1}+i}^{\prime} \quad \tilde{e}_{\lambda_{d-i+1}+i-3}^{\prime}+\tilde{e}_{\lambda_{d-i+1}+i+1}^{\prime}\right. \\
& \left.\cdots \quad \tilde{e}_{\lambda_{d-i+1}+i-d}^{\prime}+\tilde{e}_{\lambda_{d-i+1}+i+d-2}^{\prime}\right),
\end{aligned}
$$

where $\tilde{e}_{r}^{\prime}=\tilde{e}_{r}-\tilde{e}_{r-2}, \tilde{e}_{r}=\sum_{i=0}^{\infty} e_{i} e_{r+i}$ and $e_{i}=e_{i}(\mathbf{x}, \mathbf{y})$ are the elementary symmetric functions of infinitely many variables $\mathbf{x}$ and $\mathbf{y}$.

Proposition 6.2. Let $\mathbf{x}=\left\{x_{1}, x_{2}, \cdots\right\}$ be an infinite set of variables and $\mathbf{z}=$ $\left\{z_{1}, z_{2}, \cdots, z_{d}\right\}$ be $d$ variables. Then

$$
\prod_{i=1}^{d} \prod_{j=1}^{\infty}\left(1+x_{j} z_{i}\right)\left(1+x_{j} z_{i}^{-1}\right)=\sum_{\lambda} \chi_{S p(2 d)}^{\lambda}(\mathbf{z}) S_{\lambda}^{\mathfrak{s} \mathfrak{p}, d}(\mathbf{x}),
$$


where $\lambda$ runs over all partitions of length $d$, and

$$
\prod_{i=1}^{d} \prod_{j=1}^{\infty}\left(1-x_{j} z_{i}\right)^{-1}\left(1-x_{j} z_{i}^{-1}\right)^{-1}=\sum_{\lambda} \chi_{S p(2 d)}^{\lambda}(\mathbf{z}) D S_{\lambda}^{\mathfrak{s p}, d}(\mathbf{x}),
$$

where $\lambda$ runs over all partitions of length $d$.

Proof. The first identity follows from (6.3) by putting $m \rightarrow \infty$. The second identity can be obtained from the first by applying the involution of the ring of symmetric functions, which sends the elementary symmetric functions of $x_{j}$ 's to the complete symmetric functions of $x_{j}$ 's.

Proposition 6.3. Let $\mathbf{x}=\left\{x_{1}, x_{2}, \cdots\right\}$ and $\mathbf{y}=\left\{y_{\frac{1}{2}}, y_{\frac{3}{2}}, \cdots\right\}$ be two infinite sets of variables and $\mathbf{z}=\left\{z_{1}, z_{2}, \cdots, z_{d}\right\}$ be $d$ variables. Then

$$
\prod_{i=1}^{d} \prod_{j=1}^{\infty} \prod_{k=\frac{1}{2}}^{\infty} \frac{\left(1+x_{j} z_{i}\right)\left(1+x_{j} z_{i}^{-1}\right)}{\left(1-y_{k} z_{i}\right)\left(1-y_{k} z_{i}^{-1}\right)}=\sum_{\lambda} \chi_{S p(2 d)}^{\lambda}(\mathbf{z}) H S_{\lambda}^{\mathfrak{s p}, d}(\mathbf{x}, \mathbf{y}),
$$

where $\lambda$ runs over all partitions of length $d$.

Proof. By Proposition 6.2, we have

$$
\begin{aligned}
& \prod_{i=1}^{d} \prod_{j=1}^{\infty} \prod_{k=\frac{1}{2}}^{\infty}\left(1+x_{j} z_{i}\right)\left(1+x_{j} z_{i}^{-1}\right)\left(1+y_{k} z_{i}\right)\left(1+y_{k} z_{i}^{-1}\right) \\
= & \sum_{\lambda} \chi_{S p(2 d)}^{\lambda}(\mathbf{z}) S_{\lambda}^{\mathfrak{s p}, d}(\mathbf{x}, \mathbf{y})
\end{aligned}
$$

where $\lambda$ runs over all partitions of length $d$. The proposition follows by applying to both sides of equation (6.6) the involution of the ring of symmetric functions, which sends the elementary symmetric functions of $\mathbf{y}$ to the complete symmetric functions of $\mathbf{y}$.

We need the following lemma to prove the main result of this subsection.

Lemma $6.1\left([8)\right.$. Suppose that $f^{\lambda}(\mathbf{x})$ and $g^{\lambda}(\mathbf{x})$ are power series in the variables $\mathbf{x}$ and suppose that

$$
\sum_{\lambda} f^{\lambda}(\mathbf{x}) \chi_{S p(2 d)}^{\lambda}(\mathbf{z})=\sum_{\lambda} g^{\lambda}(\mathbf{x}) \chi_{S p(2 d)}^{\lambda}(\mathbf{z}),
$$

where $\lambda$ runs over all partitions of length $d$. Then $f^{\lambda}(\mathbf{x})=g^{\lambda}(\mathbf{x})$, for all $\lambda$.

Proposition 6.4. Let $\mathbf{x}=\left\{x_{1}, x_{2}, \cdots\right\}$ and $\mathbf{y}=\left\{y_{1}, y_{2}, \cdots\right\}$ be two infinite sets of variables. For each partition $\lambda$ of length $d$, we have

$$
H S_{\lambda}^{\mathfrak{s p}, d}(\mathbf{x}, \mathbf{y}):=\sum_{\mu \nu} c_{\mu \nu}^{\lambda} S_{\mu}^{\mathfrak{s p}, d}(\mathbf{x}) D S_{\nu}^{\mathfrak{s p}, d}(\mathbf{y})
$$

where $\mu$ and $\nu$ run over all partitions of length $d$. Here the non-negative integers $c_{\mu \nu}^{\lambda}$ are the multiplicity of $V_{S p(2 d)}^{\lambda}$ in the tensor product decomposition of $V_{S p(2 d)}^{\mu} \otimes$ $V_{S p(2 d)}^{\nu}$. 
Proof. By Proposition 6.2, we have

$$
\prod_{i=1}^{d} \prod_{j=1}^{\infty} \prod_{k=\frac{1}{2}}^{\infty} \frac{\left(1+x_{j} z_{i}\right)\left(1+x_{j} z_{i}^{-1}\right)}{\left(1-y_{k} z_{i}\right)\left(1-y_{k} z_{i}^{-1}\right)}=\sum_{\mu} \chi_{S p(2 d)}^{\mu}(\mathbf{z}) S_{\mu}^{\mathfrak{s} \mathfrak{p}, d}(\mathbf{x}) \sum_{\nu} \chi_{S p(2 d)}^{\nu}(\mathbf{z}) D_{\nu}^{\mathfrak{s p}, d}(\mathbf{y}),
$$

where $\mu$ and $\nu$ run over all partitions of length $d$. On the other hand,

$$
\chi_{S p(2 d)}^{\mu}(\mathbf{z}) \chi_{S p(2 d)}^{\nu}(\mathbf{z})=\sum_{\lambda} c_{\mu \nu}^{\lambda} \chi_{S p(2 d)}^{\lambda}(\mathbf{z}),
$$

where the summation is over all partitions of length $d$. Thus

$$
\begin{aligned}
& \sum_{\mu} \chi_{S p(2 d)}^{\mu}(\mathbf{z}) S_{\mu}^{\mathfrak{s p}, d}(\mathbf{x}) \sum_{\nu} \chi_{S p(2 d)}^{\nu}(\mathbf{z}) D_{\nu}^{\mathfrak{s p}, d}(\mathbf{y}) \\
= & \sum_{\lambda} \chi_{S p(2 d)}^{\lambda}(\mathbf{z})\left(\sum_{\mu \nu} c_{\mu \nu}^{\lambda} S_{\mu}^{\mathfrak{s p}, d}(\mathbf{x}) D_{\nu}^{\mathfrak{s p}, d}(\mathbf{y})\right),
\end{aligned}
$$

where $\lambda, \mu$ and $\nu$ run over all partitions of length $d$. Therefore, we have

$$
\prod_{i=1}^{d} \prod_{j=1}^{\infty} \prod_{k=\frac{1}{2}}^{\infty} \frac{\left(1+x_{j} z_{i}\right)\left(1+x_{j} z_{i}^{-1}\right)}{\left(1-y_{k} z_{i}\right)\left(1-y_{k} z_{i}^{-1}\right)}=\sum_{\lambda} \chi_{S p(2 d)}^{\lambda}(\mathbf{z})\left(\sum_{\mu \nu} c_{\mu \nu}^{\lambda} S_{\mu}^{\mathfrak{s p}, d}(\mathbf{x}) D_{\nu}^{\mathfrak{s p}, d}(\mathbf{y})\right),
$$

where $\lambda, \mu$ and $\nu$ run over all partitions of length $d$. Now the proposition follows from (6.7), Proposition 6.3 and Lemma 6.1.

Now we turn to the formal characters of unitarizable irreducible $\widehat{\mathcal{C}}$-modules. We have the following result.

Theorem 6.1. For each partition $\lambda$ of length $d$, the formal character of the irreducible $\widehat{\mathrm{e}}$-module $L\left(\widehat{\mathrm{e}}, \Lambda^{\widehat{\mathrm{e}}}(\lambda)\right)$ is given by

$$
\operatorname{ch} L\left(\widehat{\mathcal{\complement}}, \Lambda^{\widehat{\mathrm{e}}}(\lambda)\right)=H S_{\lambda}^{\mathfrak{s p}, d}(\mathbf{x}, \mathbf{y}) .
$$

Proof. One can easily show that the formal character of $\mathfrak{F}_{0}^{d}$ with respect to the abelian algebra $\sum_{s \in \frac{1}{2} \mathbb{N}} \mathbb{C} \tilde{e}_{s s} \oplus \sum_{i=1}^{d} \mathbb{C} E_{i i}$ is given by

$$
\operatorname{ch} \mathfrak{F}_{0}^{d}=\prod_{i=1}^{d} \prod_{j=1}^{\infty} \prod_{k=\frac{1}{2}}^{\infty} \frac{\left(1+x_{j} z_{i}\right)\left(1+x_{j} z_{i}^{-1}\right)}{\left(1-y_{k} z_{i}\right)\left(1-y_{k} z_{i}^{-1}\right)}
$$

By Proposition 6.3 we can rewrite (6.8) as

$$
\operatorname{ch} \mathfrak{F}_{0}^{d}=\sum_{\lambda} \chi_{S p(2 d)}^{\lambda}(\mathbf{z}) H S_{\lambda}^{\mathfrak{s}, d}(\mathbf{x}, \mathbf{y}),
$$

where $\lambda$ runs over all partitions of length $d$. On the other hand, Theorem 5.3 implies that

$$
\operatorname{ch} \mathfrak{F}_{0}^{d} \cong \sum_{\lambda} \operatorname{ch} L\left(\widehat{\mathcal{e}}, \Lambda^{\widehat{\mathrm{e}}}(\lambda)\right) \chi_{S p(2 d)}^{\lambda}(\mathbf{z}),
$$

where $\lambda$ runs over all partitions of length $d$. Using (6.10) and (6.9) together with Lemma 6.1 we obtain the claimed result for the character of the irreducible $\widehat{\mathcal{C}}$ module $L\left(\widehat{\mathcal{C}}, \Lambda^{\widehat{\mathrm{e}}}(\lambda)\right)$. 
6.2. Character formula for $\widehat{\mathcal{D}}$. We shall need results on formal characters of finite dimensional representations of the orthogonal group (see [10], 13, 14]), which we now recall.

When dealing with characters of $\mathfrak{s o}(2 m)$-modules, we let $z_{i}:=e^{\epsilon_{i}}$ where $\epsilon_{i} \in \mathfrak{h}^{*}$ such that $\epsilon_{i}\left(h_{j}\right)=\delta_{i j}$ (see Section [5.4). We define $\tilde{h}_{i}:=-h_{m-i+1}$ and $x_{i}:=$ $e^{-\epsilon_{m-i+1}}$ for $i=1,2, \cdots, m$. That is, $x_{i}=z_{m-i+1}^{-1}$. When considering characters of $O(n)$-modules, we put $z_{i}=e^{\epsilon_{i}}$ with $\epsilon_{i} \in \mathfrak{h}^{*}$ such that $\epsilon_{i}\left(h_{j}\right)=\epsilon_{i}\left(e_{j j}-e_{d+j, d+j}\right)=$ $\delta_{i j}$ for $n=2 d$, and $\epsilon_{i}\left(h_{j}\right)=\epsilon_{i}\left(e_{j j}-e_{d+j+1, d+j+1}\right)=\delta_{i j}$ for $n=2 d+1$. When we deal with characters of $\widehat{\mathcal{D}}$-modules, for $j \in \mathbb{N}, r \in \frac{1}{2}+\mathbb{Z}_{+}$, we let $x_{j}:=e^{\omega_{j}}$ and $y_{r}:=e^{\omega_{r}}$, where $\omega_{s}$ are the respective fundamental weights of $\widehat{\mathcal{D}}$ introduced in Section 4.2

Given a finite sequence of complex numbers $\lambda=\left(\lambda_{1}, \cdots, \lambda_{m}\right)$, we let $W_{\mathfrak{s o}(2 m)}^{\lambda}:=$ $V_{\mathfrak{s o}(2 m)}^{\lambda^{*}}$ where $\lambda^{*}=\left(-\lambda_{m}, \cdots,-\lambda_{1}\right)$. Note that $\lambda\left(\tilde{h}_{i}\right)=\lambda_{i}$ for $i=1, \cdots, m$. $W_{\mathfrak{s o}(2 m)}^{\lambda}$ is a finite-dimensional irreducible representation if and only if $-\left|\lambda_{1}\right| \geq$ $\lambda_{2} \geq \cdots \geq \lambda_{m}$ with either $\lambda_{i} \in \mathbb{Z}$ or $\lambda_{i} \in \frac{1}{2}+\mathbb{Z}$ for $i=2, \cdots, m$.

For each partition $\lambda$ of length $n$ and each sequence of complex numbers $\nu$ of length $m$, we write

$$
\chi_{O(n)}^{\lambda}(\mathbf{z})=\chi_{O(n)}^{\lambda}\left(z_{1}, \cdots, z_{d}\right), \quad \tilde{\chi}_{\mathfrak{s o}(2 m)}^{\nu}(\mathbf{x})=\tilde{\chi}_{\mathfrak{s o}(2 m)}^{\nu}\left(x_{1}, \cdots, x_{m}\right)
$$

for the character of $O(n)$-module $V_{O(n)}^{\lambda}$ and $\mathfrak{s o}(2 m)$-module $W_{\mathfrak{s o}(2 m)}^{\nu}$ to stress their dependence on the variables $z_{1}, \cdots, z_{d}$ and $x_{1}, \cdots, x_{m}$, respectively.

Recall that when $n=2 d+1$, the irreducible $O(n)$-modules $V_{O(n)}^{\lambda}$ and $V_{O(n)}^{\bar{\lambda}}$ restrict to isomorphic $S O(n)$-modules. To distinguish these $O(n)$-representations at the level of characters, we let $\epsilon$ be the eigenvalue of $-I_{n} \in O(n)$ so that $\epsilon^{2}=1$. Denote by $\chi_{O(n)}^{\lambda}(\epsilon, \mathbf{z})$ the character of $V_{O(n)}^{\lambda}\left(\right.$ with $\left.\lambda_{1}^{\prime}+\lambda_{2}^{\prime} \leq n\right)$ with respect to the Cartan subalgebra $\sum \mathbb{C} h_{i}$ together with $-I_{n}$. It is easy to see that

$$
\chi_{O(n)}^{\lambda}(\epsilon, \mathbf{z})=\epsilon^{|\lambda|} \chi_{S O(n)}^{\lambda}(\mathbf{z})
$$

where $|\lambda|$ is the size of $\lambda$.

By the classical $(\mathfrak{s o}(2 m), O(n))$-duality on the exterior algebra $\Lambda\left(\mathbb{C}^{n} \otimes \mathbb{C}^{m}\right)$ with $m \geq n$ (13, [14, also see [8]), we have the following identities for $n=2 d$ and $n=2 d+1$ respectively:

$$
\begin{gathered}
\left(x_{1} \cdots x_{m}\right)^{-\frac{n}{2}} \prod_{i=1}^{d} \prod_{j=1}^{m}\left(1+x_{j} z_{i}\right)\left(1+x_{j} z_{i}^{-1}\right)=\sum_{\lambda} \chi_{O(n)}^{\lambda}(\mathbf{z}) \tilde{\chi}_{\mathfrak{s o}(2 m)}^{\lambda^{\prime}-\frac{n}{2}\left(1^{m}\right)}(\mathbf{x}), \\
\left(x_{1} \cdots x_{m}\right)^{-\frac{n}{2}} \prod_{i=1}^{d} \prod_{j=1}^{m}\left(1+\epsilon x_{j} z_{i}\right)\left(1+\epsilon x_{j} z_{i}^{-1}\right)\left(1+\epsilon x_{j}\right) \\
=\sum_{\lambda} \chi_{O(n)}^{\lambda}(\epsilon, \mathbf{z}) \tilde{\chi}_{\mathfrak{s o}(2 m)}^{\lambda^{\prime}-\frac{n}{2}\left(1^{m}\right)}(\mathbf{x}) .
\end{gathered}
$$

The summations on the right-hand sides of both equations range over all partitions of length $n$ with $\lambda_{1}^{\prime}+\lambda_{2}^{\prime} \leq n$ and $\lambda_{1} \leq m$. Note that the partition $\lambda^{\prime}$ is considered as a partition of length $m$ and $\frac{n}{2} \geq \lambda_{2}^{\prime} \geq \cdots \geq \lambda_{m}^{\prime}$ together with $n \geq \lambda_{1}^{\prime}$.

Recall that

$$
E_{r}:=E_{r}\left(x_{1}, \cdots, x_{m}, x_{1}^{-1}, \cdots, x_{m}^{-1}\right)
$$


where $E_{r}$ is the $r$-th elementary symmetric polynomial for $r \geq 0$ and $E_{r}=0$ for $r<0$ (see Section 6.1). For each partition $\mu$ of length $l$, we let $\left|E_{\mu}\right|$ denote the determinant of the $l \times l$ matrix with $i$-th row

$$
\left(\begin{array}{lllll}
E_{\mu_{i}-i+1} & E_{\mu_{i}-i+2}+E_{\mu_{i}-i} & E_{\mu_{i}-i+3}+E_{\mu_{i}-i-1} & \cdots & E_{\mu_{i}-i+l}+E_{\mu_{i}-i-l+2}
\end{array}\right) .
$$

For any partition $\nu$ of length $m$ with $\nu_{m}=0$, the formal character of the finitedimensional irreducible $\mathfrak{s o}(2 m)$-module $V_{\mathfrak{s o}(2 m)}^{\nu}$ equals $\left|E_{\nu^{\prime}}\right|$. On the other hand, if the partition $\nu=\left(\nu_{1}, \cdots, \nu_{m}\right)$ is of length $m$ with $\nu_{m} \neq 0$, the characters of the finite-dimensional irreducible representations $V_{\mathfrak{s o}(2 m)}^{\nu}$ and $V_{\mathfrak{s o}(2 m)}^{\left(\nu_{1}, \cdots, \nu_{m-1},-\nu_{m}\right)}$ are respectively equal to (see [10])

$$
\frac{1}{2}\left|E_{\nu^{\prime}}\right|+\frac{1}{2}\left|E_{\left(\nu-\left(1^{m}\right)\right)^{\prime}}^{\prime}\right|\left(\prod_{i=1}^{m}\left(x_{i}-x_{i}^{-1}\right) \text { and } \frac{1}{2}\left|E_{\nu^{\prime}}\right|-\frac{1}{2}\left|E_{\left(\nu-\left(1^{m}\right)\right)^{\prime}}^{\prime}\right|\left(\prod_{i=1}^{m}\left(x_{i}-x_{i}^{-1}\right),\right.\right.
$$

where $\left|E_{\left(\nu-\left(1^{m}\right)\right)^{\prime}}^{\prime}\right|$ is the determinant of the $\left(\nu_{1}-1\right) \times\left(\nu_{1}-1\right)$ matrix defined by (6.2). Note that $\nu-\left(1^{m}\right)$ is a partition, and its transpose partition has length $\nu_{1}-1$. Also, for any partition $\nu=\left(\nu_{1}, \cdots, \nu_{m}\right)$, the characters of the finite-dimensional irreducible modules $V_{\mathfrak{s o}(2 m)}^{\nu+\frac{1}{2}\left(1^{m}\right)}$ and $V_{\mathfrak{s o}(2 m)}^{\left(\nu_{1}+\frac{1}{2}, \cdots, \nu_{m-1}+\frac{1}{2},-\nu_{m}-\frac{1}{2}\right)}$ respectively equal to

$$
\begin{aligned}
& \frac{1}{2}\left(\prod_{i=1}^{m}\left(x_{i}^{\frac{1}{2}}+x_{i}^{-\frac{1}{2}}\right)\right)\left|M_{\nu^{\prime}}^{+}\right|+\frac{1}{2}\left(\prod_{i=1}^{m}\left(x_{i}^{\frac{1}{2}}-x_{i}^{-\frac{1}{2}}\right)\right)\left|M_{\nu^{\prime}}^{-}\right|, \\
\text {and } \quad & \frac{1}{2}\left(\prod_{i=1}^{m}\left(x_{i}^{\frac{1}{2}}+x_{i}^{-\frac{1}{2}}\right)\right)\left|M_{\nu^{\prime}}^{+}\right|-\frac{1}{2}\left(\prod_{i=1}^{m}\left(x_{i}^{\frac{1}{2}}-x_{i}^{-\frac{1}{2}}\right)\right)\left|M_{\nu^{\prime}}^{-}\right| .
\end{aligned}
$$

Hereafter, we let

$$
\begin{aligned}
& \left|M_{\mu}^{-}\right|:=\left|M_{\mu}^{-}\left(x_{1}, \cdots, x_{m}, x_{1}^{-1}, \cdots, x_{m}^{-1}\right)\right|, \\
& \left|M_{\mu}^{+}\right|:=\left|M_{\mu}^{+}\left(x_{1}, \cdots, x_{m}, x_{1}^{-1}, \cdots, x_{m}^{-1}\right)\right|
\end{aligned}
$$

denote the determinants of the $l \times l$ matrices respectively having the $i$-th rows

$$
\left(\begin{array}{llll}
E_{\mu_{i}-i+1}-E_{\mu_{i}-i} & E_{\mu_{i}-i+2}-E_{\mu_{i}-i-1} & \cdots & E_{\mu_{i}-i+n}-E_{\mu_{i}-i-n+1}
\end{array}\right)
$$

and

$$
\left(\begin{array}{llll}
E_{\mu_{i}-i+1}+E_{\mu_{i}-i} & E_{\mu_{i}-i+2}+E_{\mu_{i}-i-1} & \cdots & E_{\mu_{i}-i+n}+E_{\mu_{i}-i-n+1}
\end{array}\right),
$$

where $\mu=\left(\mu_{1}, \cdots, \mu_{l}\right)$ is any partition of length $l$.

Let $\mathbf{x}=\left\{x_{1}, x_{2}, \cdots\right\}$ be an infinite set of variables. Define the orthogonal Schur function of weight $\frac{n}{2}$,

$$
S_{\lambda}^{\mathfrak{s o}, \frac{n}{2}}(\mathbf{x})=S_{\lambda}^{\mathfrak{s o}, \frac{n}{2}}\left(x_{1}, x_{2}, \cdots\right),
$$

for each partition $\lambda$ of length $n=2 d$ with $\lambda_{1}^{\prime}+\lambda_{2}^{\prime} \leq 2 d$ by

$$
S_{\lambda}^{\mathfrak{s o}, \frac{n}{2}}(\mathbf{x}):=\left\{\begin{array}{lll}
\left|\tilde{e}_{\lambda}\right|, & \text { if } & \lambda_{1}^{\prime}=\frac{n}{2} \\
\frac{1}{2}\left|\tilde{e}_{\lambda}\right|+\frac{1}{2}\left(\sum_{i=0}^{\infty} e_{i}\right)\left(\sum_{i=0}^{\infty}(-1)^{i} e_{i}\right)\left|\tilde{e}_{\lambda-\left(1^{m}\right)}\right| & \text { if } & \lambda_{1}^{\prime}<\frac{n}{2} \\
\frac{1}{2}\left|\tilde{e}_{\bar{\lambda}}\right|-\frac{1}{2}\left(\sum_{i=0}^{\infty} e_{i}\right)\left(\sum_{i=0}^{\infty}(-1)^{i} e_{i}\right)\left|\tilde{e}_{\bar{\lambda}-\left(1^{m}\right)}^{\ominus}\right| & \text { if } & \lambda_{1}^{\prime}>\frac{n}{2},
\end{array}\right.
$$

where $\left|\tilde{e}_{\lambda}\right|$ denotes the determinant of the $d \times d$ matrix whose $i$-th row is

$$
\begin{aligned}
& \left(\begin{array}{lll}
\tilde{e}_{\lambda_{d-i+1}+i-1} & \tilde{e}_{\lambda_{d-i+1}+i-2}+\tilde{e}_{\lambda_{d-i+1}+i} & \tilde{e}_{\lambda_{d-i+1}+i-3}+\tilde{e}_{\lambda_{d-i+1}+i+1}
\end{array}\right. \\
& \left.\cdots \tilde{e}_{\lambda_{d-i+1}+i-d}+\tilde{e}_{\lambda_{d-i+1}+i+d-2}\right),
\end{aligned}
$$


and $\left|\tilde{e}_{\lambda}^{\diamond}\right|$ denotes the determinant of the $(d-1) \times(d-1)$ matrix whose $i$-th row is

$$
\begin{aligned}
& \left(\begin{array}{lll}
\tilde{e}_{\lambda_{d-i}+i-1}^{\prime} & \tilde{e}_{\lambda_{d-i}+i-2}^{\prime}+\tilde{e}_{\lambda_{d-i}+i}^{\prime} & \tilde{e}_{\lambda_{d-i}+i-3}^{\prime}+\tilde{e}_{\lambda_{d-i}+i+1}^{\prime}
\end{array}\right. \\
& \text { ․ } \tilde{e}_{\lambda_{d-i}+i-d}^{\prime}+\tilde{e}_{\lambda_{d-i}+i+d-2}^{\prime} \text { ). }
\end{aligned}
$$

Here $\tilde{e}_{r}=\sum_{i=0}^{\infty} e_{i}(\mathbf{x}) e_{r+i}(\mathbf{x}), \tilde{e}_{r}^{\prime}=\tilde{e}_{r}-\tilde{e}_{r-2}$ and $e_{i}(\mathbf{x})$ is the $i$-th elementary symmetric function in the infinite set of variables $\mathbf{x}$. Recall that $\bar{\lambda}$ is a partition of length $n$ obtained from the Young diagram of $\lambda$ by replacing its first column by a column of length $n-\lambda_{1}^{\prime}$ (see Section 5.4).

Similarly, for each partition $\lambda$ of length $n=2 d+1$ with $\lambda_{1}^{\prime}+\lambda_{2}^{\prime} \leq 2 d+1$, the orthogonal Schur function of weight $\frac{n}{2}$,

$$
S_{\lambda}^{\mathfrak{s o}, \frac{n}{2}}(\mathbf{x})=S_{\lambda}^{\mathfrak{s o}, \frac{n}{2}}\left(x_{1}, x_{2}, \cdots\right),
$$

in infinitely many variables is defined by

$$
S_{\lambda}^{\mathfrak{s o}, \frac{n}{2}}(\mathbf{x}):= \begin{cases}\frac{1}{2}\left(\sum_{i=0}^{\infty} e_{i}\right)\left|\tilde{m}_{\lambda}^{+}\right|+\frac{1}{2}\left(\sum_{i=0}^{\infty}(-1)^{i} e_{i}\right)\left|\tilde{m}_{\lambda}^{-}\right| & \text {if } \quad \lambda_{1}^{\prime} \leq \frac{n}{2} ; \\ \frac{1}{2}\left(\sum_{i=0}^{\infty} e_{i}\right)\left|\tilde{m}_{\bar{\lambda}}^{+}\right|-\frac{1}{2}\left(\sum_{i=0}^{\infty}(-1)^{i} e_{i}\right)\left|\tilde{m}_{\bar{\lambda}}^{-}\right| & \text {if } \quad \lambda_{1}^{\prime}>\frac{n}{2},\end{cases}
$$

where $\left|\tilde{m}_{\lambda}^{+}\right|$denotes the determinant of the $d \times d$ matrix whose $i$-th row is

$$
\left.\begin{array}{rl}
\left(\tilde{e}_{\lambda_{d-i+1}+i-1}+\tilde{e}_{\lambda_{d-i+1}+i}\right. & \tilde{e}_{\lambda_{d-i+1}+i-2}+\tilde{e}_{\lambda_{d-i+1}+i+1} \\
\cdots & \tilde{e}_{\lambda_{d-i+1}+i-d}+\tilde{e}_{\lambda_{d-i+1}+i+d-1}
\end{array}\right),
$$

and $\left|\tilde{m}_{\lambda}^{-}\right|$denotes the determinant of the $d \times d$ matrix whose $i$-th row is

$$
\left.\begin{array}{rl}
\left(\tilde{e}_{\lambda_{d-i+1}+i-1}-\tilde{e}_{\lambda_{d-i+1}+i}\right. & \tilde{e}_{\lambda_{d-i+1}+i-2}-\tilde{e}_{\lambda_{d-i+1}+i+1} \\
\cdots & \tilde{e}_{\lambda_{d-i+1}+i-d}-\tilde{e}_{\lambda_{d-i+1}+i+d-1}
\end{array}\right) .
$$

We put $S_{\lambda}^{\mathfrak{s o}, d}\left(x_{1}, \cdots, x_{m}\right)=S_{\lambda}^{\mathfrak{s o}, d}\left(x_{1}, \cdots, x_{m}, 0,0, \cdots\right)$. Using similar arguments as for $\mathfrak{s p}(2 m)$ in Section 6.1, we have

$$
\left(x_{1} \cdots x_{m}\right)^{\frac{n}{2}} \tilde{\chi}_{\mathfrak{s o}(2 m)}^{\lambda^{\prime}-d\left(1^{m}\right)}\left(x_{1}, \cdots, x_{m}\right)=S_{\lambda}^{\mathfrak{s o}, d}\left(x_{1}, \cdots, x_{m}\right)
$$

for any partition $\lambda$ of length $n$ with $\lambda_{1}^{\prime}+\lambda_{2}^{\prime} \leq n$ and $\lambda_{1} \leq m$. Now we can rewrite the combinatorial formulae (6.11) and (6.12) respectively as follows (for $m \geq n$ ):

$$
\prod_{i=1}^{d} \prod_{j=1}^{m}\left(1+x_{j} z_{i}\right)\left(1+x_{j} z_{i}^{-1}\right)=\sum_{\lambda} \chi_{O(n)}^{\lambda}(\mathbf{z}) S_{\lambda}^{\mathfrak{s o}, \frac{n}{2}}\left(x_{1}, \cdots, x_{m}\right)
$$

for even integer $n=2 d$ and

$$
\prod_{i=1}^{d} \prod_{j=1}^{m}\left(1+\epsilon x_{j} z_{i}\right)\left(1+\epsilon x_{j} z_{i}^{-1}\right)\left(1+\epsilon x_{j}\right)=\sum_{\lambda} \chi_{O(n)}^{\lambda}(\epsilon, \mathbf{z}) S_{\lambda}^{\mathfrak{s o}, \frac{n}{2}}\left(x_{1}, \cdots, x_{m}\right),
$$

for odd integer $n=2 d+1$. In both equations $\lambda$ runs over all partitions of length $n$ satisfying $\lambda_{1}^{\prime}+\lambda_{2}^{\prime} \leq n$ and $\lambda_{1} \leq m$.

Let $\mathbf{x}=\left\{x_{1}, x_{2}, \cdots\right\}$ and $\mathbf{y}=\left\{y_{\frac{1}{2}}, y_{1 \frac{1}{2}}, \cdots\right\}$ be two infinite sets of variables. Corresponding to each partition $\lambda$ of length $n$, we define the skew orthogonal Schur function $D_{\lambda}^{\mathfrak{s o}, \frac{n}{2}}(\mathbf{x})=D_{\lambda}^{\mathfrak{s o}, \frac{n}{2}}\left(x_{1}, x_{2}, \cdots\right)$ of weight $\frac{n}{2}$ of infinitely many variables by $D_{\lambda}^{\mathfrak{s o}, \frac{n}{2}}(\mathbf{x})=\sigma\left(D_{\lambda}^{\mathfrak{s o}, \frac{n}{2}}(\mathbf{x})\right)$, where $\sigma$ is the involution of the ring of symmetric functions sending the elementary symmetric functions of $x_{j}$ 's to the complete symmetric functions of $x_{j}$ 's. Also, for each partition $\lambda$ of length $n$, the hook orthogonal 
Schur function $H S_{\lambda}^{\mathfrak{s o}, \frac{n}{2}}(\mathbf{x}, \mathbf{y})$ of weight $\frac{n}{2}$ of infinitely many variables is defined by $\theta\left(S_{\lambda}^{\mathfrak{s o}, \frac{n}{2}}(\mathbf{x}, \mathbf{y})\right)$, where $\theta$ is the involution of the ring of symmetric functions sending the elementary symmetric functions of $y_{j}$ 's to the complete symmetric functions of $y_{j}$ 's.

By using 6.13), 6.14) and similar arguments as in Section 6.1, we can prove the following two propositions.

Proposition 6.5. Let $\mathbf{x}=\left\{x_{1}, x_{2}, \cdots\right\}$ be an infinite set of variables and let $\mathbf{z}=\left\{z_{1}, z_{2}, \cdots, z_{d}\right\}$ be d variables.

(i) When $n=2 d$, we have

$$
\begin{aligned}
& \prod_{i=1}^{d} \prod_{j=1}^{\infty}\left(1+x_{j} z_{i}\right)\left(1+x_{j} z_{i}^{-1}\right)=\sum_{\lambda} \chi_{O(n)}^{\lambda}(\mathbf{z}) S_{\lambda}^{\mathfrak{s o}, \frac{n}{2}}(\mathbf{x}), \\
& \prod_{i=1}^{d} \prod_{j=1}^{\infty}\left(1-x_{j} z_{i}\right)^{-1}\left(1-x_{j} z_{i}^{-1}\right)^{-1}=\sum_{\lambda} \chi_{O(n)}^{\lambda}(\mathbf{z}) D_{\lambda}^{\mathfrak{s o}, \frac{n}{2}}(\mathbf{x}),
\end{aligned}
$$

where the summations on the right-hand sides of both equations range over all partitions of length $n$ satisfying $\lambda_{1}^{\prime}+\lambda_{2}^{\prime} \leq n$.

(ii) When $n=2 d+1$, we have

$$
\begin{aligned}
& \prod_{i=1}^{d} \prod_{j=1}^{\infty}\left(1+\epsilon x_{j} z_{i}\right)\left(1+\epsilon x_{j} z_{i}^{-1}\right)\left(1+\epsilon x_{j}\right)=\sum_{\lambda} \chi_{O(n)}^{\lambda}(\epsilon, \mathbf{z}) S_{\lambda}^{\mathfrak{s o}, \frac{n}{2}}(\mathbf{x}) \\
& \prod_{i=1}^{d} \prod_{j=1}^{\infty}\left(1-\epsilon x_{j} z_{i}\right)^{-1}\left(1-\epsilon x_{j} z_{i}^{-1}\right)^{-1}\left(1-\epsilon x_{j}\right)^{-1}=\sum_{\lambda} \chi_{O(n)}^{\lambda}(\epsilon, \mathbf{z}) D_{\lambda}^{\mathfrak{s o}, \frac{n}{2}}(\mathbf{x}),
\end{aligned}
$$

where the summations on the right-hand sides of both equations range over all partitions of length $n$ satisfying $\lambda_{1}^{\prime}+\lambda_{2}^{\prime} \leq n$.

Proposition 6.6. Let $\mathbf{x}=\left\{x_{1}, x_{2}, \cdots\right\}$ and $\mathbf{y}=\left\{y_{\frac{1}{2}}, y_{1 \frac{1}{2}}, \cdots\right\}$ be two infinite sets of variables and $\mathbf{z}=\left\{z_{1}, z_{2}, \cdots, z_{d}\right\}$ be $d$ variables.

(i) When $n=2 d$, we have

$$
\prod_{i=1}^{d} \prod_{j=1}^{\infty} \prod_{k=\frac{1}{2}}^{\infty} \frac{\left(1+x_{j} z_{i}\right)\left(1+x_{j} z_{i}^{-1}\right)}{\left(1-y_{k} z_{i}\right)\left(1-y_{k} z_{i}^{-1}\right)}=\sum_{\lambda} \chi_{O(n)}^{\lambda}(\mathbf{z}) H S_{\lambda}^{\mathfrak{s o}, \frac{n}{2}}(\mathbf{x}, \mathbf{y})
$$

where $\lambda$ runs over all partitions of length $n$.

(ii) When $n=2 d+1$, we have

$$
\prod_{i=1}^{d} \prod_{j=1}^{\infty} \prod_{k=\frac{1}{2}}^{\infty} \frac{\left(1+\epsilon x_{j} z_{i}\right)\left(1+\epsilon x_{j} z_{i}^{-1}\right)\left(1+\epsilon x_{j}\right)}{\left(1-\epsilon y_{k} z_{i}\right)\left(1-\epsilon y_{k} z_{i}^{-1}\right)\left(1-\epsilon y_{k}\right)}=\sum_{\lambda} \chi_{O(n)}^{\lambda}(\epsilon, \mathbf{z}) H S_{\lambda}^{\mathfrak{s o}, \frac{n}{2}}(\mathbf{x}, \mathbf{y}),
$$

where $\lambda$ runs over all partitions of length $n$.

We need the following results to prove Theorem 6.2

Lemma $6.2([8])$. Let $f^{\lambda}(\mathbf{y})$ and $g^{\lambda}(\mathbf{y})$ be power series in the variables $\mathbf{y}$.

(i) Suppose that $n$ is odd and

$$
\sum_{\lambda} f^{\lambda}(\mathbf{y}) \chi_{O(n)}^{\lambda}(\epsilon, \mathbf{x})=\sum_{\lambda} g^{\lambda}(\mathbf{y}) \chi_{O(n)}^{\lambda}(\epsilon, \mathbf{x}),
$$


where the summation is over all partitions of length $n$. Then $f^{\lambda}(\mathbf{y})=g^{\lambda}(\mathbf{y})$, for all $\lambda$.

(ii) Suppose that $n$ is even and

$$
\sum_{\lambda} f^{\lambda}(\mathbf{y}) \chi_{O(n)}^{\lambda}(\mathbf{x})=\sum_{\lambda} g^{\lambda}(\mathbf{y}) \chi_{O(n)}^{\lambda}(\mathbf{x}),
$$

where the summation is over all partitions of length $n$. Then $f^{\lambda}(\mathbf{y})+$ $f^{\bar{\lambda}}(\mathbf{y})=g^{\lambda}(\mathbf{y})+g^{\bar{\lambda}}(\mathbf{y})$.

Proposition 6.7. Let $\mathbf{x}=\left\{x_{1}, x_{2}, \cdots\right\}$ and $\mathbf{y}=\left\{y_{1}, y_{2}, \cdots\right\}$ be two sets of infinitely many variables. Let $n$ be a fixed non-negative integer and let $b_{\mu \nu}^{\lambda}$ denote the multiplicity of $V_{O(n)}^{\lambda}$ in the tensor product decomposition of $V_{O(n)}^{\mu} \otimes V_{O(n)}^{\nu}$.

(i) Suppose that $n$ is odd, for any partition $\lambda$ of length $n$ with $\lambda_{1}^{\prime}+\lambda_{2}^{\prime} \leq n$, we have

$$
H S_{\lambda}^{\mathfrak{s o}, \frac{n}{2}}(\mathbf{x}, \mathbf{y})=\sum_{\mu, \nu} b_{\mu \nu}^{\lambda} S_{\mu}^{\mathfrak{s o}, \frac{n}{2}}(\mathbf{x}) D S_{\nu}^{\mathfrak{s o}, \frac{n}{2}}(\mathbf{y})
$$

where $\mu$ and $\nu$ run over all partitions of length $n$.

(ii) Suppose that $n$ is even, for any partition of length $n=$ with $\lambda_{1}^{\prime}+\lambda_{2}^{\prime} \leq n$, we have

$$
\begin{aligned}
& H S_{\lambda}^{\mathfrak{s o}, \frac{n}{2}}(\mathbf{x}, \mathbf{y})+H S_{\bar{\lambda}}^{\mathfrak{s o}, \frac{n}{2}}(\mathbf{x}, \mathbf{y}) \\
= & \left(\sum_{\mu, \nu} b_{\mu \nu}^{\lambda} S_{\mu}^{\mathfrak{s o}, \frac{n}{2}}(\mathbf{x}) D_{\nu}^{\mathfrak{s o}, \frac{n}{2}}(\mathbf{y})\right)+\left(\sum_{\mu, \nu} b_{\mu \nu}^{\bar{\lambda}} S_{\mu}^{\mathfrak{s o}, \frac{n}{2}}(\mathbf{x}) D_{\nu}^{\mathfrak{s o}, \frac{n}{2}}(\mathbf{y})\right),
\end{aligned}
$$

where $\mu$ and $\nu$ run over all partitions of length $n$ satisfying $\mu_{1}^{\prime}+\mu_{2}^{\prime} \leq n$ and $\nu_{1}^{\prime}+\nu_{2}^{\prime} \leq n$, respectively.

Proof. We shall only prove the case with $n$ being even, as the odd case is analogous. Let $n=2 d$. By Proposition 6.5, we have

$$
\prod_{i=1}^{d} \prod_{j=1}^{\infty} \prod_{k=\frac{1}{2}}^{\infty} \frac{\left(1+x_{j} z_{i}\right)\left(1+x_{j} z_{i}^{-1}\right)}{\left(1-y_{k} z_{i}\right)\left(1-y_{k} z_{i}^{-1}\right)}=\sum_{\mu} \chi_{O(n)}^{\mu}(\mathbf{z}) S_{\mu}^{\mathfrak{s o}, \frac{n}{2}}(\mathbf{x}) \sum_{\nu} \chi_{O(n)}^{\nu}(\mathbf{z}) D_{\nu}^{\mathfrak{s o}, \frac{n}{2}}(\mathbf{y})
$$

where $\mu$ and $\nu$ run over all partitions of length $n$ satisfying $\lambda_{1}^{\prime}+\lambda_{2}^{\prime} \leq n$ and $\mu_{1}^{\prime}+\mu_{2}^{\prime} \leq n$, respectively. On the other hand,

$$
\chi_{O(n)}^{\mu}(\mathbf{z}) \chi_{O(n)}^{\nu}(\mathbf{z})=\sum_{\lambda} b_{\mu \nu}^{\lambda} \chi_{O(n)}^{\lambda}(\mathbf{z}),
$$

where $\lambda$ runs over all partitions of length $n$ with $\lambda_{1}^{\prime}+\lambda_{2}^{\prime} \leq n$. Thus

$$
\begin{aligned}
& \sum_{\mu} \chi_{O(n)}^{\mu}(\mathbf{z}) S_{\mu}^{\mathfrak{s o}, \frac{n}{2}}(\mathbf{x}) \sum_{\nu} \chi_{O(n)}^{\nu}(\mathbf{z}) D_{\nu}^{\mathfrak{s o}, \frac{n}{2}}(\mathbf{y}) \\
= & \sum_{\lambda} \chi_{O(n)}^{\lambda}(\mathbf{z})\left(\sum_{\mu, \nu} b_{\mu \nu}^{\lambda} S_{\mu}^{\mathfrak{s o}, \frac{n}{2}}(\mathbf{x}) D_{\nu}^{\mathfrak{s o}, \frac{n}{2}}(\mathbf{y})\right),
\end{aligned}
$$

where $\lambda, \mu$ and $\nu$ run over all partitions of length $n$ satisfying $\lambda_{1}^{\prime}+\lambda_{2}^{\prime} \leq n, \mu_{1}^{\prime}+\mu_{2}^{\prime} \leq$ $n$ and $\nu_{1}^{\prime}+\nu_{2}^{\prime} \leq n$, respectively. Therefore, we have

$$
\prod_{i=1}^{d} \prod_{j=1}^{\infty} \prod_{k=\frac{1}{2}}^{\infty} \frac{\left(1+x_{j} z_{i}\right)\left(1+x_{j} z_{i}^{-1}\right)}{\left(1-y_{k} z_{i}\right)\left(1-y_{k} z_{i}^{-1}\right)}=\sum_{\lambda} \chi_{O(n)}^{\lambda}(\mathbf{z})\left(\sum_{\mu \nu} b_{\mu \nu}^{\lambda} S_{\mu}^{\mathfrak{s o}, \frac{n}{2}}(\mathbf{x}) D_{\nu}^{\mathfrak{s o}, \frac{n}{2}}(\mathbf{y})\right)
$$


where $\lambda, \mu$ and $\nu$ run over all partitions of length $n$ satisfying $\lambda_{1}^{\prime}+\lambda_{2}^{\prime} \leq n, \mu_{1}^{\prime}+\mu_{2}^{\prime} \leq$ $n$ and $\nu_{1}^{\prime}+\nu_{2}^{\prime} \leq n$, respectively. Now by using Proposition 6.6, equation (6.19) and Lemma 6.2. for any partition of length $n$ with $\lambda_{1}^{\prime}+\lambda_{2}^{\prime} \leq n$, we have

$$
\begin{aligned}
& H S_{\lambda}^{\mathfrak{s o}, \frac{n}{2}}(\mathbf{x}, \mathbf{y})+H S_{\bar{\lambda}}^{\mathfrak{s o}, \frac{n}{2}}(\mathbf{x}, \mathbf{y}) \\
= & \left(\sum_{\mu, \nu} b_{\mu \nu}^{\lambda} S_{\mu}^{\mathfrak{s o}, \frac{n}{2}}(\mathbf{x}) D_{\nu}^{\mathfrak{s o}, \frac{n}{2}}(\mathbf{y})\right)+\left(\sum_{\mu, \nu} b_{\mu \nu}^{\bar{\lambda}} S_{\mu}^{\mathfrak{s o}, \frac{n}{2}}(\mathbf{x}) D_{\nu}^{\mathfrak{s o}, \frac{n}{2}}(\mathbf{y})\right),
\end{aligned}
$$

where $\mu$ and $\nu$ run over all partitions of length $n$ satisfying $\mu_{1}^{\prime}+\mu_{2}^{\prime} \leq n$ and $\nu_{1}^{\prime}+\nu_{2}^{\prime} \leq n$, respectively.

Now we consider the formal characters of the Fock spaces. Let $d=\left[\frac{n}{2}\right]$, that is, $n=2 d$ if $n$ is even and $n=2 d+1$ if $n$ is odd. We can easily show that the character of $\mathfrak{F}_{0}^{d}$, with respect to the abelian algebra $\sum_{s \in \frac{1}{2} \mathbb{N}} \mathbb{C} \tilde{e}_{s s} \oplus \sum_{i=1}^{d} \mathbb{C} E_{i i}$, is given by

$$
\operatorname{ch} \mathfrak{F}_{0}^{d}=\prod_{i=1}^{d} \prod_{j=1}^{\infty} \prod_{k=\frac{1}{2}}^{\infty} \frac{\left(1+x_{j} z_{i}\right)\left(1+x_{j} z_{i}^{-1}\right)}{\left(1-y_{k} z_{i}\right)\left(1-y_{k} z_{i}^{-1}\right)}
$$

Similarly, the character of $\mathfrak{F}_{0}^{d+\frac{1}{2}}$, with respect to the abelian algebra $\sum_{s \in \frac{1}{2} \mathbb{N}} \mathbb{C} \tilde{e}_{s s} \oplus$ $\sum_{i=1}^{d} \mathbb{C} E_{i i}$, is given by

$$
\operatorname{ch} \mathfrak{F}_{0}^{d+\frac{1}{2}}=\prod_{i=1}^{d} \prod_{j=1}^{\infty} \prod_{k=\frac{1}{2}}^{\infty} \frac{\left(1+\epsilon x_{j} z_{i}\right)\left(1+\epsilon x_{j} z_{i}^{-1}\right)\left(1+\epsilon x_{j}\right)}{\left(1-\epsilon y_{k} z_{i}\right)\left(1-\epsilon y_{k} z_{i}^{-1}\right)\left(1-\epsilon y_{k}\right)} .
$$

By Proposition 6.6, we can rewrite (6.20) in the case of even $n$ as

$$
\operatorname{ch} \mathfrak{F}_{0}^{\frac{n}{2}}=\sum_{\lambda} \chi_{O(n)}^{\lambda}(\mathbf{z}) H S_{\lambda}^{\mathfrak{s o}, \frac{n}{2}}(\mathbf{x}, \mathbf{y}),
$$

where $\lambda$ runs over all partitions of length $n$ with $\lambda_{1}^{\prime}+\lambda_{2}^{\prime} \leq n$. On the other hand, Theorem 5.4 implies that

$$
\operatorname{ch} \mathfrak{F}_{0}^{\frac{n}{2}}=\sum_{\lambda} \operatorname{ch} L\left(\widehat{\mathcal{D}}, \Lambda^{\widehat{\mathcal{D}}}(\lambda)\right) \chi_{O(n)}^{\lambda}(\mathbf{z}),
$$

where $\lambda$ runs over all partitions of length $n$ with $\lambda_{1}^{\prime}+\lambda_{2}^{\prime} \leq n$. Combining (6.23) with (6.22), we arrive at

$$
\sum_{\lambda} \chi_{O(n)}^{\lambda}(\mathbf{z}) H S_{\lambda}^{\mathfrak{s o}, \frac{n}{2}}(\mathbf{x}, \mathbf{y})=\sum_{\lambda} \operatorname{ch} L\left(\widehat{\mathcal{D}}, \Lambda^{\widehat{\mathcal{D}}}(\lambda)\right) \chi_{O(n)}^{\lambda}(\mathbf{z}) .
$$

In a similar way, we can also derive the following equation for $n$ odd:

$$
\sum_{\lambda} \chi_{O(n)}^{\lambda}(\epsilon, \mathbf{z}) H S_{\lambda}^{\mathfrak{s o}, \frac{n}{2}}(\mathbf{x}, \mathbf{y})=\sum_{\lambda} \operatorname{ch} L\left(\widehat{\mathcal{D}}, \Lambda^{\widehat{\mathcal{D}}}(\lambda)\right) \chi_{O(n)}^{\lambda}(\epsilon, \mathbf{z}) .
$$

Applying Lemma 6.2 to these equations, we obtain the following character formulae:

Theorem 6.2. For each partition $\lambda$ of length $n=2 d+1$ with $\lambda_{1}^{\prime}+\lambda_{2}^{\prime} \leq n$, we have

$$
\operatorname{ch} L\left(\widehat{\mathcal{D}}, \Lambda^{\widehat{\mathcal{D}}}(\lambda)\right)=H S_{\lambda}^{\mathfrak{s o}, \frac{n}{2}}(\mathbf{x}, \mathbf{y}) .
$$

For each partition $\lambda$ of length $n=2 d$ with $\lambda_{1}^{\prime}+\lambda_{2}^{\prime} \leq n$, we have

$$
\operatorname{ch} L\left(\widehat{\mathcal{D}}, \Lambda^{\widehat{\mathcal{D}}}(\lambda)\right)+\operatorname{ch} L\left(\widehat{\mathcal{D}}, \Lambda^{\widehat{\mathcal{D}}}(\bar{\lambda})\right)=H S_{\lambda}^{\mathfrak{s o}, \frac{n}{2}}(\mathbf{x}, \mathbf{y})+H S_{\bar{\lambda}}^{\mathfrak{s o}, \frac{n}{2}}(\mathbf{x}, \mathbf{y}) .
$$


Remark 6.1. From Theorem 6.1 and Theorem 6.2 we can easily extract character formulae for the unitarizable quasi-finite irreducible highest weight modules over the $\mathfrak{s o}$ and $\mathfrak{s p}$ type subalgebras of $\widehat{\mathrm{gl}}_{\infty}$.

\section{ACKNOWLEDGEMEnTS}

We thank Shun-Jen Cheng for discussions. Financial support from the Australian Research Council and the National Science Council of the Republic of China is gratefully acknowledged.

\section{REFERENCES}

[1] Berele, A.; Regev, A.: Hook Young diagrams with applications to combinatorics and representations of Lie superalgebras, Adv. Math. 64 (1987) 118-175. MR0884183(88i:20006)

[2] Bröcker, T; tom Dieck, T.: Representations of compact Lie Groups, Springer-Verlag, New York 1995. MR 1410059 (97i:22005)

[3] Cheng, S.-J.; Lam, N.: Infinite-dimensional Lie superalgebras and hook Schur functions, Commun. Math. Phys., 238 (2003) 95-118. MR1989670 (2004e:17021)

[4] Cheng, S.-J.; Lam, N.; Zhang, R.B.: Character Formula for Infinite Dimensional Unitarizable Modules of the General Linear Superalgebra, J. Algebra 273 (2004), 780-805. MR 2037723 (2004j:17009)

[5] Cheng, S.-J.; Wang, W.: Howe Duality for Lie Superalgebras, Compositio Math. 128 (2001) 55-94. MR:1847665 (2002h:17005)

[6] Cheng, S.-J.; Wang, W.: Remarks on the Schur-Howe-Sergeev Duality, Lett. Math. Phys. 52 (2000) 143-153. MR 1786858 (2002f:17008)

[7] Cheng, S.-J.; Wang, W.: Lie subalgebras of differential operators on the super circle, Publ. RIMS, 39 (2003) 545-600. MR2001187 (2004f:17032)

[8] Cheng, S.-J.; Zhang, R. B.: Howe Duality and Combinatorial Character Formula for Orthosymplectic Lie superalgebras, Adv. Math., 182 (2004), 124-172. MR2028498

[9] Frenkel, E.; Kac, V.; Radul, A.; Wang, W.: $W_{1+\infty}$ and $W\left(g l_{N}\right)$ with central charge $N$, Comm. Math. Phys. 170 (1995) 337-357. MR.1334399 (96i:17024)

[10] Fulton, W.; Harris, J.: Representation Theory: a first course, Springer-Verlag, New York 1991. MR 1153249 (93a:20069)

[11] Goodman, R.; Wallach, N.: Representations and Invariants of the Classical Groups, Cambridge University Press, Cambridge, 1998. MR1606831 (99b:20073)

[12] Hasegawa, K.: Spin module versions of Weyl's reciprocity theorem for classical Kac-Moody Lie algebras - an application to branching rule duality, Publ. Res. Inst. Math. Sci. 25 (1989) 741-828. MR 1031225 (91f:17023)

[13] Howe, R.: Remarks on classical invariant theory, Trans. Amer. Math. Soc. 313 (1989) 539-570. MR0986027 (90h:22015a)

[14] Howe, R.: Perspectives on Invariant Theory: Schur Duality, Multiplicity-free Actions and Beyond, The Schur Lectures, Israel Math. Conf. Proc. 8, Tel Aviv (1992) 1-182. MR.1321638 (96e:13006)

[15] Kac, V.: Lie superalgebras, Adv. Math. 26 (1977) 8-96. MR0486011 (58:5803)

[16] Kac, V.: Infinite dimensional Lie algebras, Cambridge University Press, Cambridge, 1990. MR 1104219 (92k:17038)

[17] Kac, V.; Radul, A.: Quasi-finte highest weight modules over the Lie algebra of differential operators on the circle, Commun. Math. Phys. 157 (1993) 429-457. MR1243706|(95f:81036)

[18] Kac, V.; Wang, W.; Yan, C.: Quasi-finite representations of classical Lie subalgebras of $W_{1+\infty}$, Adv. Math. 139 (1998) 56-140. MR1652526 (2000g:17039)

[19] Macdonald, I. G.: Symmetric functions and Hall polynomials, Oxford Math. Monogr., Clarendon Press, Oxford, 1995. MR1354144 (96h:05207)

[20] Nazarov, M.: Capelli identities for Lie superalgebras, Ann. Scient. Éc Norm. Sup. $4^{e}$ série 30 (1997) 847-872. MR:1476298 (99b:17008)

[21] Ol'shanskii, G.; Prati, M., Extremal weights of finite-dimensional representations of the Lie superalgebra $\mathfrak{g} l_{n \mid m}$, Il Nuovo Cimento, 85 A (1985) 1-18. MR0789738(87e:17008) 
[22] Sergeev, A.: An analog of the classical invariant theory for Lie superalgebras, I, Michigan Math. J. 49 (2001) 113-146. MR.1827078 (2003d:17008)

[23] Sergeev, A.: An analog of the classical invariant theory for Lie superalgebras, II, Michigan Math. J. 49 (2001) 147-168. MR.1827078 (2003d:17008)

[24] Wang, W.: Duality in infinite dimensional Fock representations, Comm. Contem. Math. 1 (1999) 155-199. MR1696098 (2000j:17030)

[25] Wang, W.: Dual Pairs and Infinite Dimensional Lie Algebras, In: Recent Developments in Quantum Affine Algebras and Related Topics, (eds. N. Jing and K.C. Misra), Contemp. Math. 248 (1999) 453-469. MR.1745273 (2001h:17029)

Department of Mathematics, National Cheng Kung University, Tainan, Taiwan 701

E-mail address: nlam@mail.ncku.edu.tw

School of Mathematics and Statistics, University of Sydney, New South Wales 2006, Australia

E-mail address: rzhang@maths.usyd.edu.au 